\title{
2012 Annual Summary Report for the Area 3 and Area 5 Radioactive Waste Management Sites at the Nevada National Security Site Nye County, Nevada
}

\section{Review of the Performance Assessments and Composite Analyses}

March 2013

Prepared by

National Security Technologies, LLC

Las Vegas, Nevada

\section{National Security Technologies LLc}

Prepared for

U.S. Department of Energy, National Nuclear Security Administration

Nevada Site Office

Under Contract Number

DE-AC52-06NA25946 


\title{
DISCLAIMER
}

Reference herein to any specific commercial product, process, or service by trade name, trademark, manufacturer, or otherwise does not necessarily constitute or imply its endorsement, recommendation, or favoring by the U.S. Government or any agency thereof or its contractors or subcontractors.

Available for sale to the public, in paper, from:

\section{U.S. Department of Commerce}

National Technical Information Service

5301 Shawnee Road

Alexandria, VA 22312

Phone: (800) 553-6847

Fax: (703) 605-6900

E-mail: orders@ntis.gov

Online Ordering: http://www.ntis.gov/help/ordermethods.aspx

Available electronically at http://www.osti.gov/bridge

Available for a processing fee to the U.S. Department of Energy and its contractors, in paper, from:

\author{
U.S. Department of Energy \\ Office of Scientific and Technical Information \\ P.O. Box 62 \\ Oak Ridge, TN 37831-0062 \\ Phone: (865) 576-8401 \\ Fax: (865) 576-5728 \\ E-mail: reports@adonis.osti.gov
}




\title{
2012 Annual Summary Report for the Area 3 and Area 5 Radioactive Waste Management Sites at the Nevada National Security Site Nye County, Nevada
}

\section{Review of the Performance Assessments and Composite Analyses}

March 2013

\author{
Prepared by \\ National Security Technologies, LLC \\ Las Vegas, Nevada
}

Prepared for

U.S. Department of Energy, National Nuclear Security Administration

Nevada Site Office Under Contract Number

DE-AC52-06NA25946 
This Page Intentionally Left Blank 


\section{EXECUTIVE SUMMARY}

The Maintenance Plan for the Performance Assessments and Composite Analyses for the Area 3 and Area 5 Radioactive Waste Management Sites at the Nevada Test Site (National Security Technologies, LLC 2007a) requires an annual review to assess the adequacy of the performance assessments (PAs) and composite analyses (CAs), with the results submitted to the U.S. Department of Energy (DOE) Office of Environmental Management. The Disposal Authorization Statements for the Area 3 and Area 5 Radioactive Waste Management Sites (RWMSs) also require that such reviews be made and that secondary or minor unresolved issues be tracked and addressed as part of the maintenance plan (DOE 1999a, 2000).

The U.S. Department of Energy, National Nuclear Security Administration Nevada Site Office performed an annual review of the Area 3 and Area 5 RWMS PAs and CAs for fiscal year (FY) 2012. This annual summary report presents data and conclusions from the FY 2012 review, and determines the adequacy of the PAs and CAs. Operational factors (e.g., waste forms and containers, facility design, and waste receipts), closure plans, monitoring results, and research and development (R\&D) activities were reviewed to determine the adequacy of the PAs. Likewise, the environmental restoration activities at the Nevada National Security Site (NNSS) relevant to the sources of residual radioactive material that are considered in the CAs, the land-use planning, and the results of the environmental monitoring and R\&D activities were reviewed to determine the adequacy of the CAs.

Important developments in FY 2012 include the following:

- Release of a special analysis for the Area 3 RWMS assessing the continuing validity of the PA and CA

- Development of a new Area 5 RWMS closure inventory estimate based on disposals through FY 2012

- Evaluation of new or revised waste streams by special analysis

- Development of version 4.114 of the Area 5 RWMS GoldSim PA model

The Area 3 RWMS has been in inactive status since July 1, 2006, with the last shipment received in April 2006. The FY 2012 review of operations, facility design, closure plans, monitoring results, and R\&D results for the Area 3 RWMS indicates no changes that would impact PA validity. A special analysis using the Area 3 RWMS v2.102 GoldSim PA model was prepared to update the PA results for the Area 3 RWMS in FY 2012. The special analysis concludes that all performance objectives can be met and the Area 3 RWMS PA remains valid. There is no need to the revise the Area 3 RWMS PA.

Review of Area 5 RWMS operations, design, closure plans, monitoring results, and R\&D activities indicates no significant changes other than an increase in the inventory disposed. The FY 2012 PA results, generated with the Area 5 RWMS v4.114 GoldSim PA model, indicate that there continues to be a reasonable expectation of meeting all performance objectives. The results 
and conclusions of the Area 5 RWMS PA are judged valid, and there is no need to the revise the PA.

A review of changes potentially impacting the CAs indicates that no significant changes occurred in FY 2012. The continuing adequacy of the CAs was evaluated with the new models, and no significant changes that would alter CA results or conclusions were found. The revision of the Area 3 RWMS CA, which will include the Underground Test Area source term (Corrective Action Unit [CAU] 97), is scheduled for FY 2024, following the completion of the Yucca Flat CAU 97 Corrective Action Decision Document/Corrective Action Plan in FY 2016. Inclusion of the Frenchman Flat CAU 98 results in the Area 5 RWMS CA is scheduled for FY 2016, pending the completion of the CAU 98 closure report in FY 2015.

Near-term R\&D efforts will focus on continuing development of the Area 3 and Area 5 RWMS GoldSim PA/CA and inventory models. 


\section{TABLE OF CONTENTS}

EXECUTIVE SUMMARY

ACRONYMS AND ABBREVIATIONS ............................................................................ vii

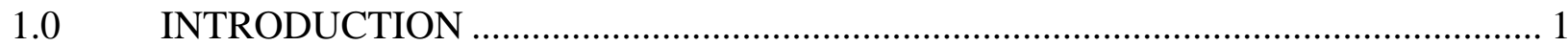

1.1 Status of Disposal Authorization Statement Conditions............................................. 2

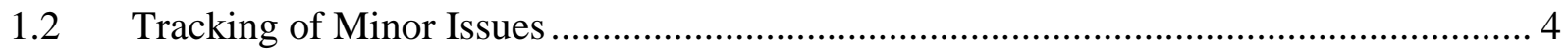

2.0 PERFORMANCE ASSESSMENT REVIEW …................................................. 7

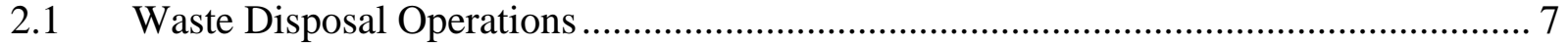

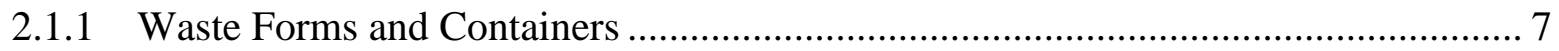

2.1.2 Waste Receipts......................................................................................... 7

2.1.2.1 New or Revised Waste Streams ......................................................................... 7

2.1.2.2 FY 2012 Closure Inventory Estimate for the Area 3 RWMS ............................ 8

2.1.2.3 FY 2012 Closure Inventory Estimate for the Area 5 RWMS ......................... 13

2.1.3 Waste Acceptance Criteria................................................................................ 22

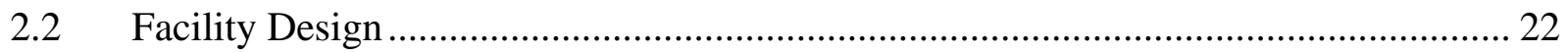

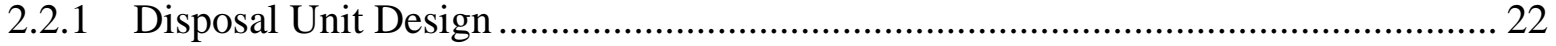

2.2.2 Engineered Barriers ............................................................................... 23

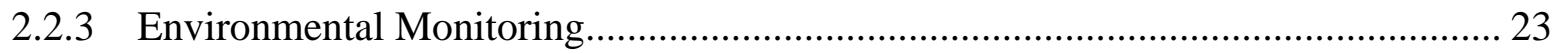

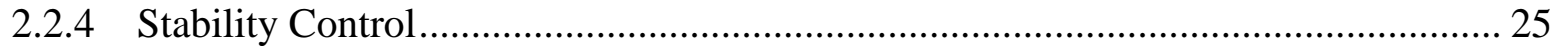

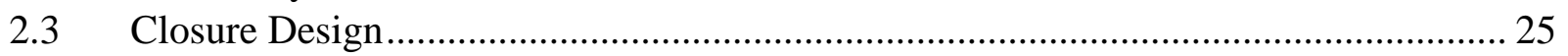

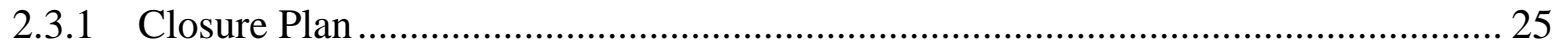

2.3.2 Institutional Control Policy .............................................................................. 26

$2.4 \quad$ Research and Development................................................................................. 27

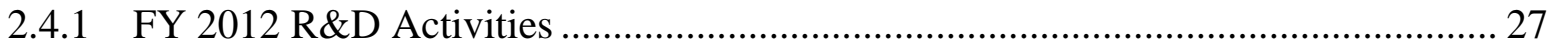

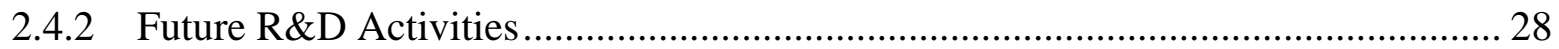

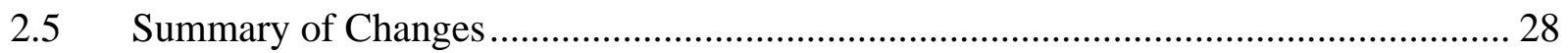

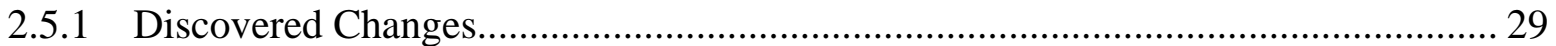

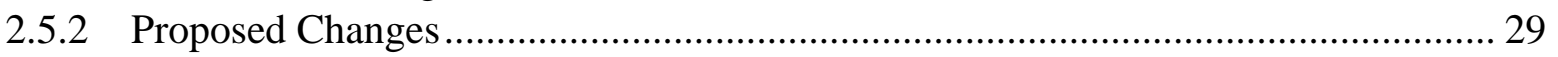

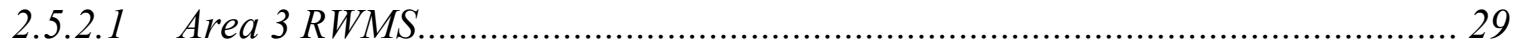

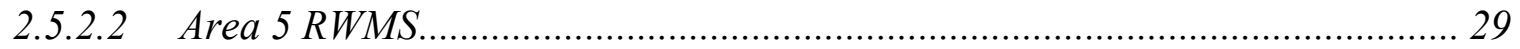

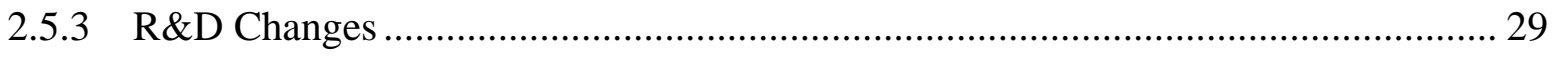

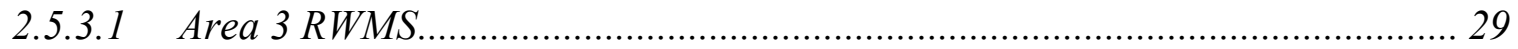

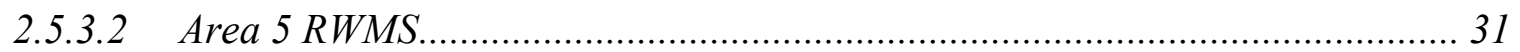

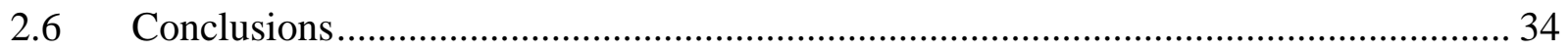

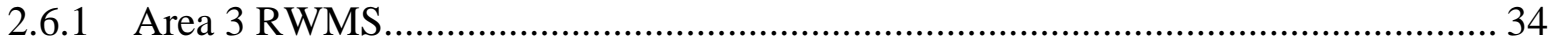

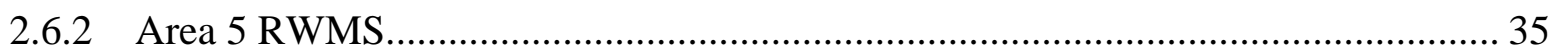

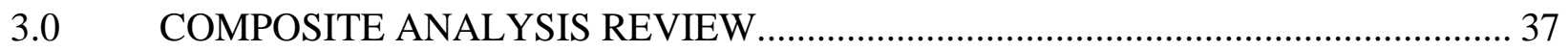

3.1 Waste Operations and Environmental Remediation ............................................... 37

3.1.1 Radioactive Waste Management Sites.......................................................... 37

3.1.1.1 Waste Characteristics and Facility Design ................................................ 37 


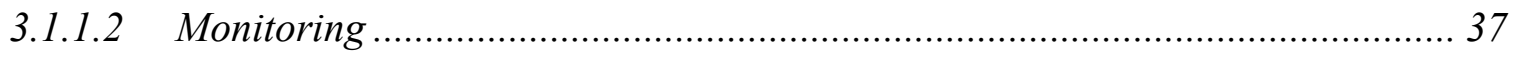

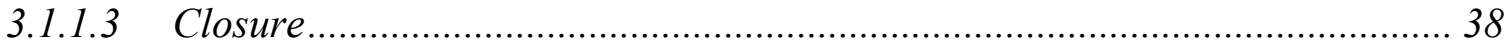

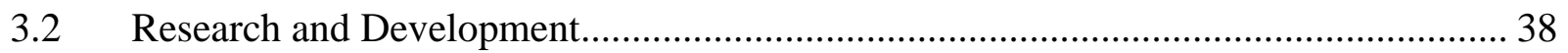

Interacting Source Terms ................................................................................... 38

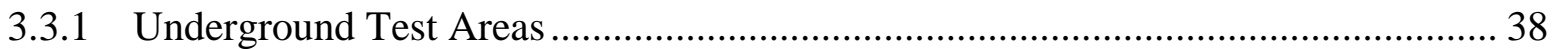

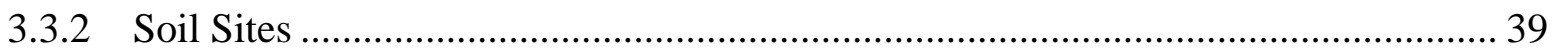

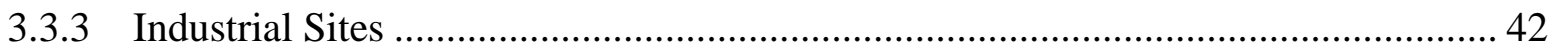

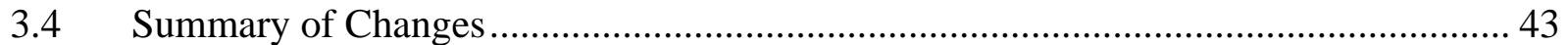

3.4.1 Discovered Changes................................................................................... 43

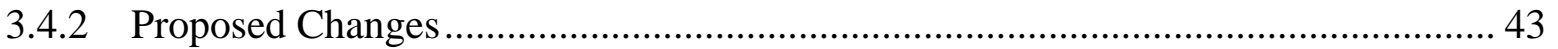

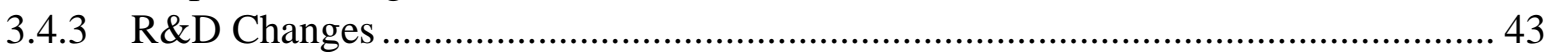

3.4.3.1 CA Results for the Area 3 RWMS ................................................................ 43

3.4.3.2 CA Results for the Area 5 RWMS .................................................................. 44

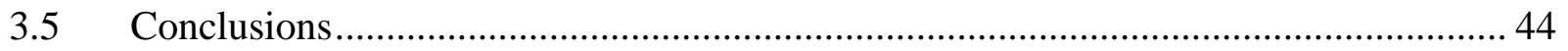

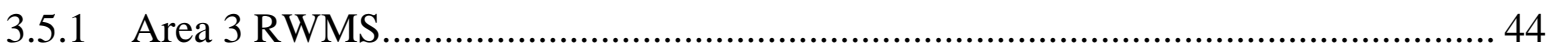

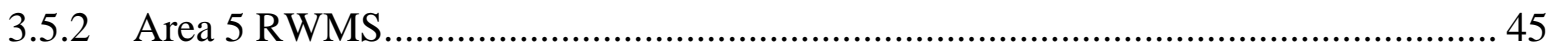

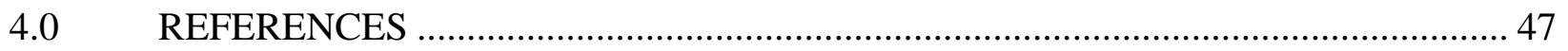

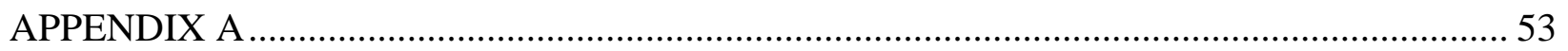

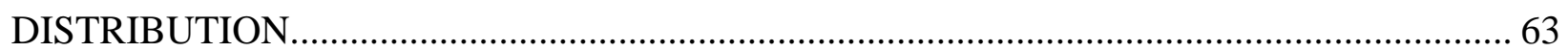




\section{List of Figures}

Figure 1. Annual Volume Disposal Rate and Total Volume for the Area 3 RWMS ................. 13

Figure 2. Activity Annual Disposal Rate and Total Inventory for the Area 3 RWMS................ 13

Figure 3. Annual Volume Disposal Rate and Total Volume for the Area 5 RWMS SLB Disposal

Units.... 17

Figure 4. Activity Annual Disposal Rate and Total Inventory for the Area 5 RWMS SLB Disposal Units 18

\section{List of Tables}

Table 1. Status of the Area 3 RWMS DAS PA Conditions...................................................... 2

Table 2. Status of the Area 3 RWMS DAS CA Conditions .................................................. 3

Table 3. Status of the Area 5 RWMS DAS PA Conditions......................................................... 3

Table 4. Status of the Area 5 RWMS DAS CA Conditions ................................................... 4

Table 5. Minor Issues Identified in the LFRG Review Reports for the Area 3 and Area 5 RWMS

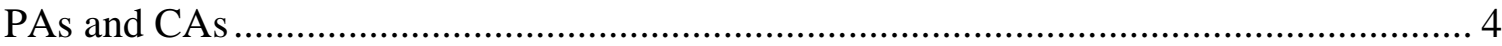

Table 6. Waste Streams Evaluated by Special Analysis in FY 2012 ..................................... 8

Table 7. FY 2012 Estimate of the Area 3 RWMS Inventory Disposed before September 26, 1988

Table 8. FY 2012 Estimate of the Area 3 RWMS Inventory Disposed after September 26, 1988

Table 9. FY 2012 Estimate of the Area 5 RWMS SLB Inventory ............................................ 14

Table 10. FY 2012 Estimate of the Area 5 RWMS RaDU Inventory Disposed ......................... 19

Table 11. FY 2012 Estimate of the Area 5 RWMS GCD Borehole Inventory .......................... 20

Table 12. Summary of Area 3 and Area 5 RWMS Monitoring Programs ................................. 24

Table 13. Area 3 RWMS v2.102 GoldSim Model Member of Public Annual TED through the Air Pathway ........................................................................................................... 30

Table 14. Area 3 RWMS v2.102 GoldSim Model Member of Public Annual TED through All-

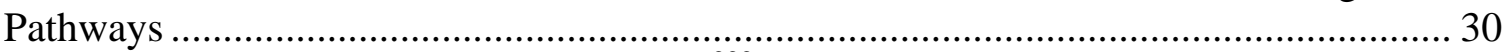

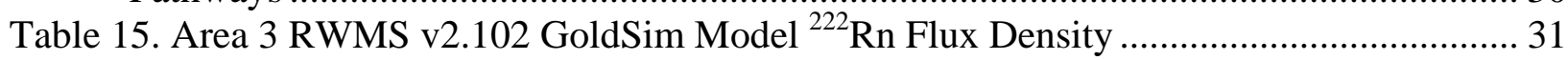

Table 16. Area 3 RWMS v2.102 GoldSim Model Acute Drilling Intruder TED........................ 31

Table 17. Area 3 RWMS v2.102 GoldSim Model Acute Construction Intruder ........................ 31

Table 18. Area 5 RWMS v4.114 GoldSim Model Member of Public Annual TED through the

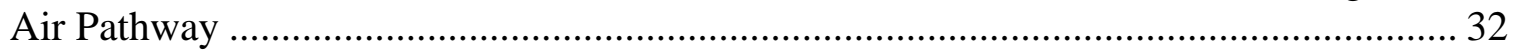

Table 19. Area 5 RWMS v4.114 GoldSim Model Member of Public Annual TED through All-

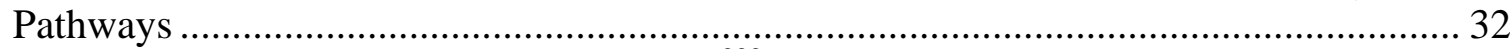

Table 20. Area 5 RWMS v4.114 GoldSim Model ${ }^{222}$ Rn Flux Density Results .......................... 33

Table 21. Area 5 RWMS v4.114 GoldSim Model Acute Drilling Intruder TED....................... 33

Table 22. Area 5 RWMS v4.114 GoldSim Model Acute Construction Intruder TED................. 34

Table 23. Status of ER Soil Sites Considered in the Area 3 RWMS CA ................................. 40

Table 24. Status of ER Soil Sites Considered in the Area 5 RWMS CA .................................. 41

Table 25. Area 3 RWMS v2.102 GoldSim Model CA All-Pathways Annual TED for a Resident

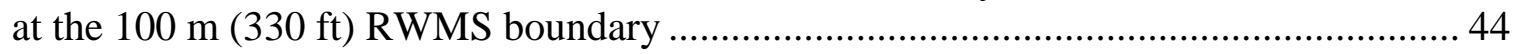


Table 26. Area 5 RWMS v4.114 GoldSim Model CA All-Pathways Annual TED for a Resident

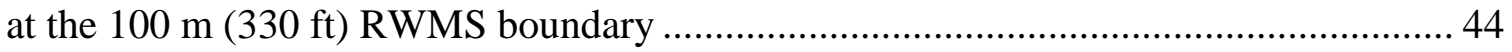

Table A.1. Checklist for Review of Annual Summary ............................................................ 53 


\section{ACRONYMS AND ABBREVIATIONS}

ac

$\mathrm{Am}$

$\mathrm{BN}$

$\mathrm{Bq}$

$\mathrm{Bq} \mathrm{m} \mathrm{m}^{-2} \mathrm{~s}^{-1}$

BUP

CA

CADD

CAIP

CAP

CAS

CAU

CFR

$\mathrm{Ci}$

Co

COCs

COPCs

CR

CY

DAS

DOE

DU

EPA

ER

ET

FEPs

FFACO

$\mathrm{ft}$

$\mathrm{ft}^{3}$

FY

GCD

GMX

GZ

${ }^{3} \mathrm{H}$

ISC3

acre

americium

Bechtel Nevada

becquerel

becquerel per square meter per second

Byproduct Utilization Program

composite analysis

Corrective Action Decision Document

Corrective Action Investigation Plan

Corrective Action Plan

Corrective Action Site

Corrective Action Unit

Code of Federal Regulations

curie

cobalt

contaminants of concern

contaminants of potential concern

Closure Report

calendar year

Disposal Authorization Statement

U.S. Department of Energy

depleted uranium

U.S. Environmental Protection Agency

Environmental Restoration

evapotranspirative

features, events, and processes

Federal Facility Agreement and Consent Order

foot

cubic foot

fiscal year

Greater Confinement Disposal

Gadget, Mechanics, and Explosives

ground zero

tritium

Industrial Source Code 3 


\section{ACRONYMS AND ABBREVIATIONS (continued)}

$\begin{array}{ll}\text { LFRG } & \text { Low-Level Waste Disposal Facility Federal Review Group } \\ \text { LHS } & \text { Latin hypercube sampling } \\ \text { LLW } & \text { low-level waste } \\ \text { LLWMU } & \text { Low-Level Waste Management Unit } \\ \mathrm{m} & \text { meter } \\ \text { MFP } & \text { mixed fission products } \\ \mathrm{m}^{3} & \text { cubic meter } \\ \text { mSv } & \text { millisievert } \\ \text { NDEP } & \text { Nevada Division of Environmental Protection } \\ \text { NNSA/NSO } & \text { U.S. Department of Energy, National Nuclear Security Administration Nevada } \\ \text { NNSS } & \text { Site Office } \\ \text { NSTec } & \text { Nevada National Security Site } \\ \text { PA } & \text { pational Security Technologies, LLC } \\ \text { Pb } & \text { lead } \\ \text { PSM } & \text { potential source material } \\ \text { Pu } & \text { plutonium } \\ \text { R\&D } & \text { research and development } \\ \text { Ra } & \text { radium } \\ \text { RaDU } & \text { radium disposal unit } \\ \text { RCRA } & \text { Resource Conservation and Recovery Act } \\ \text { RIDP } & \text { Radionuclide Inventory and Distribution Program } \\ \text { Rn } & \text { radon } \\ \text { RTG } & \text { radioisotope thermoelectric generator } \\ \text { RWAP } & \text { Radioactive Waste Acceptance Program } \\ \text { RWMS } & \text { Radioactive Waste Management Site } \\ \text { SLB } & \text { shallow land burial } \\ \text { Sr } & \text { strontium } \\ \text { TBD } & \text { to be determined } \\ \text { TBq } & \text { terabecquerel } \\ \text { Tc } & \text { technetium } \\ \text { TED } & \text { total effective dose } \\ \text { Th } & \text { thorium } \\ \text { TLD } & \text { thermoluminescent dosimeter } \\ \text { TRU } & \text { transuranic } \\ \text { U } & \text { uranium } \\ \text { UGTA } & \text { Underground Test Area } \\ \text { WAC } & \text { waste acceptance criteria } \\ & \end{array}$




\subsection{INTRODUCTION}

This report summarizes the results and conclusions of an annual review of the Area 3 and Area 5 Radioactive Waste Management Sites (RWMSs) performance assessments (PAs) and composite analyses (CAs). The Area 3 and Area 5 RWMSs were issued Disposal Authorization Statements (DASs) in accordance with U.S. Department of Energy Order DOE O 435.1 "Radioactive Waste Management” (U.S. Department of Energy [DOE] 2001). The Area 3 RWMS and Area 5 RWMS DASs (DOE 1999a, 2000) require preparation of an annual summary report and a determination of the continuing adequacy of the PAs and CAs. The requirement to prepare an annual summary report is implemented in the Maintenance Plan for the PAs and CAs (National Security Technologies, LLC [NSTec] 2007a). The annual summary report is submitted to the DOE Office of Environmental Management for review and approval.

The annual review summarizes changes in site operations, facility design, site monitoring, research and development $(\mathrm{R} \& \mathrm{D}), \mathrm{PA} / \mathrm{CA}$ models, and planning documents that may impact the validity of the PA and CA. The impact of changes and new information on the adequacy of the PA and CA is evaluated by answering three key questions:

1. Does the annual summary information indicate that changes to the PA or CA are required?

2. Does the annual summary information indicate that the conclusions of the PA and CA remain valid?

3. Does the annual summary information indicate that facility performance will remain within the U.S. Department of Energy Manual DOE M 435.1-1, "Radioactive Waste Management” (DOE 1999b) PA performance objectives, CA performance goals, and any conditions in the facility DAS?

This report follows the format in U.S. Department of Energy Guide DOE G 435.1-4, "Maintenance Guide for U.S. Department of Energy Low-Level Waste Disposal Facility Performance Assessments and Composite Analysis” (DOE 1999c) and presents the annual summary for the PAs in Section 2.0 and the CAs in Section 3.0. The annual summary for the PAs includes the following:

- Section 2.1 summarizes changes in waste disposal operations and includes new estimates of the closure inventories derived from the actual disposals through fiscal year (FY) 2012.

- Section 2.2 summarizes changes related to facility design and environmental monitoring.

- $\quad$ Section 2.3 summarizes closure plans and land use plans.

- Section 2.4 summarizes R\&D activities conducted under the U.S. Department of Energy, National Nuclear Security Administration Nevada Site Office (NNSA/NSO) Closure and Monitoring Plans for the Area 3 and Area 5 RWMSs (NSTec 2007b, 2008a).

- Section 2.5 is a summary of changes, including proposed and discovered changes, in facility design, operation, future plans, the monitoring plan, $R \& D$ activities, and the maintenance program. 
- Section 2.6 answers the key review questions addressing the continuing validity of the PA.

Section 3.0 presents the annual summary for the CAs emphasizing changes not addressed in the PA annual summary. The annual summary for the CAs includes the following:

- Section 3.1 presents an assessment of activities at the Nevada National Security Site (NNSS) that would impact the sources of residual radioactive material considered in the CAs.

- Section 3.2 summarizes R\&D results for FY 2012.

- Section 3.3 updates the status of sources of residual radioactive material interacting with the Area 3 and Area 5 RWMSs.

- Section 3.4 summarizes changes in monitoring plans, R\&D activities, and the maintenance program that occurred since the CAs were prepared.

- Section 3.4.3.1 updates the CA results using the FY 2012 inventories and models.

- Section 3.5 answers the key review questions regarding the continuing validity of the CA.

Appendix A is a self evaluation using the Low-Level Waste Disposal Facility Federal Review Group (LFRG) annual summary checklist.

\subsection{STATUS OF DISPOSAL AUTHORIZATION STATEMENT CONDITIONS}

The Area 3 RWMS PA and CA were issued in a single document (Shott et al. 2001). The Area 3 RWMS was issued a DAS on October 20, 1999 (DOE 1999a). The Area 3 RWMS DAS contained one PA condition and two CA conditions (Tables 1 and 2). The DAS conditions were resolved with the PA/CA document revision (Shott et al. 2001).

Table 1. Status of the Area 3 RWMS DAS PA Conditions

\begin{tabular}{|l|l|}
\hline \multicolumn{1}{|c|}{ Condition } & \multicolumn{1}{|c|}{ Status } \\
\hline "Provide to LFRG, within eight months of the date of issuance of & A revised Area 3 RWMS PA/CA \\
this disposal authorization statement, a revision to the performance & was issued in December 2001 \\
assessment that includes resolution of the following secondary & (Shott et al. 2001). The DAS \\
issues: 1) Lack of justification for excluding particular exposure & conditions were closed in 2002 \\
scenarios based on exhumed waste, 2) Inadequate justification for & (DOE 2002a). \\
omission of surface water, 3) Lack of sensitivity analysis regarding & \\
the assumed 250 years of institutional control, 4) Need for & \\
clarification of the Resource Conservation and Recovery Act & \\
(RCRA)/CERCLA regulatory involvement, if any, in low-level waste & \\
disposal at Area 3, 5) Need for clarification of the location of the & \\
point of maximum exposure, 6) Need for better explanation of the & \\
borehole and field data within the framework of the no-recharge & \\
conceptual model." & \\
\hline
\end{tabular}


Table 2. Status of the Area 3 RWMS DAS CA Conditions

\begin{tabular}{|l|l|}
\hline \multicolumn{1}{|c|}{ Condition } & \multicolumn{1}{|c|}{ Status } \\
\hline $\begin{array}{l}\text { "Provide to LFRG, within eight months of the date of issuance of } \\
\text { this disposal authorization statement, a revision to the composite } \\
\text { analysis that includes qualitative assessment including an options } \\
\text { analysis of the effect of groundwater contamination resulting from } \\
\text { underground nuclear testing. Before any portion of the Nevada Test } \\
\begin{array}{l}\text { Site is considered for a reduction in institutional control, Nevada } \\
\text { Operations Office will have quantified the potential dose from the } \\
\text { underground testing residues and taken measures to mitigate the } \\
\text { dose, as appropriate." }\end{array}\end{array}$ & $\begin{array}{l}\text { A revised Area 3 RWMS PA/CA } \\
\text { was issued in December 2001 } \\
\text { (Shott et al. 2001). The DAS } \\
\text { conditions were closed in 2002 } \\
\text { (DOE 2002a). }\end{array}$ \\
\hline $\begin{array}{l}\text { "Resolution of the following secondary issues identified in the } \\
\text { review of the composite analysis: Need for a better explanation of } \\
\text { the borehole and field data within the framework of the no-recharge } \\
\text { conceptual model." }\end{array}$ & $\begin{array}{l}\text { A revised Area 3 RWMS PA/CA } \\
\text { was issued in December 2001 } \\
\text { (Shott et al. 2001). The DAS } \\
\text { conditions were closed in 2002 } \\
\text { (DOE 2002a). }\end{array}$ \\
\hline
\end{tabular}

The Area 5 RWMS PA documentation consists of the original DOE O 435.1 low-level waste (LLW) PA (Shott et al. 1998) and supporting addenda (Bechtel Nevada [BN] 2001a, 2006). The Area 5 RWMS CA was issued as a single document (BN 2001b) and has a single addendum (BN 2001c).

In addition to the LLW PA, a PA was prepared and approved to meet the requirements of Title 40 Code of Federal Regulations (CFR) Part 191, “Environmental Radiation Protection Standards for Management and Disposal of Spent Nuclear Fuel, High-Level, and Transuranic Radioactive Waste” (CFR 1994). The 40 CFR 191 PA was prepared for transuranic (TRU) waste disposed in Greater Confinement Disposal (GCD) boreholes at the Area 5 RWMS (Cochran et al. 2001).

The Area 5 RWMS DAS was issued on December 5, 2000 (DOE 2000). The PA and CA each had two conditions (Tables 3 and 4). The DAS conditions were closed on May 23, 2002.

Table 3. Status of the Area 5 RWMS DAS PA Conditions

\begin{tabular}{|c|c|}
\hline Condition & Status \\
\hline $\begin{array}{l}\text { "The specific radionuclide concentration or inventory limits shall be } \\
\text { imposed on Pit } 6 \text { to ensure that performance objectives will not be } \\
\text { exceeded. A quantitative dose estimate shall be calculated using } \\
\text { the reduced inventory to determine compliance with the } \\
\text { performance objective." }\end{array}$ & $\begin{array}{l}\text { An addendum to the Area } 5 \\
\text { RWMS PA was issued in } \\
\text { November } 2001 \text { (BN 2001a). The } \\
\text { DAS conditions were closed in } \\
2002 \text { (DOE 2002b). }\end{array}$ \\
\hline $\begin{array}{l}\text { "The closure plan shall require a closure cap thickness of at least } \\
4 \text { meters as stated in Section } 5.1 \text { of the } 1998 \text { PA to ensure that } \\
\text { performance objectives for the agricultural scenario will not be } \\
\text { exceeded. A quantitative dose estimate shall be calculated using } \\
\text { the } 4 \text { meter cap to demonstrate compliance with the performance } \\
\text { objectives." }\end{array}$ & $\begin{array}{l}\text { An addendum to the Area } 5 \\
\text { RWMS PA was issued in } \\
\text { November } 2001 \text { (BN 2001a). The } \\
\text { DAS conditions were closed in } \\
2002 \text { (DOE 2002b). }\end{array}$ \\
\hline
\end{tabular}


Table 4. Status of the Area 5 RWMS DAS CA Conditions

\begin{tabular}{|c|c|}
\hline Condition & Status \\
\hline $\begin{array}{l}\text { "The CA for the RWMS shall either be revised or an addendum } \\
\text { issued within one year of the date of the issuance of this DAS to } \\
\text { incorporate the Supplemental Information. The revised CA or } \\
\text { addendum shall be submitted to the LFRG. Nevada Operations } \\
\text { Office shall address all secondary issues and issues identified in } \\
\text { Appendix B of the Review Team Report through the maintenance } \\
\text { program." }\end{array}$ & $\begin{array}{l}\text { An addendum to the Area } 5 \\
\text { RWMS CA was issued in } \\
\text { November } 2001 \text { (BN 2001c). The } \\
\text { DAS conditions were closed in } \\
2002 \text { (DOE 2002b). }\end{array}$ \\
\hline $\begin{array}{l}\text { "Consistent with the site's Land-Use Plan and the conditions } \\
\text { identified in the Area } 3 \text { DAS before any portion of the Nevada Test } \\
\text { Site is considered for a reduction in institutional controls, Nevada } \\
\text { Operations Office will have quantified the potential dose from the } \\
\text { underground testing residues." }\end{array}$ & $\begin{array}{l}\text { An addendum to the Area } 5 \\
\text { RWMS CA was issued in } \\
\text { November } 2001 \text { (BN 2001c). The } \\
\text { DAS conditions were closed in } \\
2002 \text { (DOE 2002b). }\end{array}$ \\
\hline
\end{tabular}

\subsection{TRACKING OF MINOR ISSUES}

Tracking and resolution of all minor or secondary issues identified in the LFRG review reports for the Area 3 and Area 5 RWMS PAs and CAs are accomplished through the current Maintenance Plan (NSTec 2007a). The Maintenance Plan is in final form. Table 5 lists the minor issues that are being tracked and resolved through the maintenance program. The resolution pathway for each issue is included in the third column of Table 5.

Table 5. Minor Issues Identified in the LFRG Review Reports for the Area 3 and Area 5 RWMS PAs and CAs

\begin{tabular}{|c|c|c|}
\hline Identified Issue & $\begin{array}{l}\text { Source } \\
\text { Document for } \\
\text { Issue }\end{array}$ & Resolution Pathway \\
\hline $\begin{array}{l}\text { An engineered barrier will be } \\
\text { added, and the assurance } \\
\text { requirements of } 40 \text { CFR } 191 \text { must } \\
\text { be met for the GCD boreholes. }\end{array}$ & GCD PA & $\begin{array}{l}\text { An engineered barrier will be added, and the } \\
\text { assurance requirements will be met at the } \\
\text { time of final closure of the Area } 5 \text { RWMS in } \\
\text { FY } 2028 \text {. }\end{array}$ \\
\hline $\begin{array}{l}\text { Inconsistencies exist between } \\
\text { conceptual models for the Area } 5 \\
\text { RWMS PA and CA, the Area } 3 \\
\text { RWMS PA and CA, and the GCD } \\
\text { PA. }\end{array}$ & $\begin{array}{l}\text { Area } 5 \text { RWMS } \\
\text { PA, Area } 5 \\
\text { RWMS CA, } \\
\text { Area } 3 \text { RWMS } \\
\text { PA/CA, GCD PA }\end{array}$ & $\begin{array}{l}\text { The continuous development of probabilistic } \\
\text { performance assessment models using the } \\
\text { GoldSim software system is systematically } \\
\text { eliminating inconsistencies; this work will } \\
\text { continue to be described in annual summary } \\
\text { reports. }\end{array}$ \\
\hline $\begin{array}{l}\text { Conduct site monitoring and site } \\
\text { characterization studies, as } \\
\text { required, to increase confidence in } \\
\text { the results of the PAs. }\end{array}$ & $\begin{array}{l}\text { Area } 3 \text { RWMS } \\
\text { PA/CA }\end{array}$ & $\begin{array}{l}\text { Monitoring programs at both Area } 5 \text { and } \\
\text { Area } 3 \text { RWMSs are ongoing; data are being } \\
\text { incorporated into the GoldSim models to } \\
\text { increase confidence in the PA results. }\end{array}$ \\
\hline $\begin{array}{l}\text { The maintenance program must } \\
\text { include periodic assessment of } \\
\text { changes in potentially interacting } \\
\text { sources (Underground Test Areas } \\
\text { [UGTAs], industrial sites) and } \\
\text { impacts on the CAs. }\end{array}$ & $\begin{array}{l}\text { Area } 5 \text { RWMS } \\
\text { CA, Area } 3 \\
\text { RWMS PA/CA }\end{array}$ & $\begin{array}{l}\text { Changes in potentially interacting sources will } \\
\text { be evaluated through the maintenance } \\
\text { program, and results will be presented in the } \\
\text { annual summary reports. }\end{array}$ \\
\hline
\end{tabular}




\begin{tabular}{|l|l|l|}
\hline \multicolumn{1}{|c|}{ Identified Issue } & \multicolumn{1}{|c|}{$\begin{array}{c}\text { Source } \\
\text { Document for } \\
\text { Issue }\end{array}$} & \multicolumn{1}{c|}{ Resolution Pathway } \\
\hline $\begin{array}{l}\text { The maintenance program must } \\
\text { include periodic assessment of } \\
\text { changes in land-use restrictions } \\
\text { and impacts on the CAs. }\end{array}$ & $\begin{array}{l}\text { Area 5 RWMS } \\
\text { CA, Area 3 } \\
\text { RWMS PA/CA }\end{array}$ & $\begin{array}{l}\text { Changes in land-use restrictions will be } \\
\text { reviewed through the maintenance program, } \\
\text { and results will be presented in the annual } \\
\text { summary reports. }\end{array}$ \\
\hline $\begin{array}{l}\text { Monitoring systems need to be } \\
\text { deployed and data gathered and } \\
\text { evaluated to distinguish between } \\
\text { interacting sources at the Area 3 } \\
\text { RWMS. }\end{array}$ & $\begin{array}{l}\text { Area 3 RWMS } \\
\text { PA/CA }\end{array}$ & $\begin{array}{l}\text { The monitoring systems deployed at the } \\
\text { disposal facilities are described in the site } \\
\text { closure plans (NSTec 2007b, 2008a); } \\
\text { monitoring results will be evaluated and } \\
\text { presented in the annual summary reports. }\end{array}$ \\
\hline
\end{tabular}


This Page Intentionally Left Blank 


\subsection{PERFORMANCE ASSESSMENT REVIEW}

The PA maintenance plan requires an annual review of waste operations including evaluation of waste forms, waste containers, facility design, waste acceptance criteria (WAC), closure design, and waste inventory. Changes in waste inventory, facility design, WAC, environmental monitoring, institutional controls, and closure design occurring during FY 2012 are noted and described below. The impacts of these changes are assessed in Section 2.5.

\subsection{WASTE DISPOSAL OPERATIONS}

\subsubsection{Waste Forms and Containers}

The Area 3 and Area 5 RWMS PAs do not explicitly model the effects of waste forms and containers on the near-field release of radionuclides. Radionuclides are assumed to be fully available for release and transport at site closure. These assumptions continue to apply for waste disposed at the Area 3 and Area 5 RWMSs through FY 2012.

\subsubsection{Waste Receipts}

The Area 3 and Area 5 RWMS PAs analyze waste inventories that are estimated as the sum of known past disposals and estimated future disposals. The closure inventory estimate changes over time as records of past disposals are revised, waste received do not match forecasts, or future waste forecasts change. Closure inventory uncertainty is dominated by uncertainty in future disposals. Sources of uncertainty that are unique to future disposals include approval of new generators or new waste streams and wastes being sent to alternative disposal sites. The FY 2012 closure inventory estimates for the Area 3 and Area 5 RWMS are summarized below.

\subsubsection{New or Revised Waste Streams}

Each new or revised waste stream is evaluated by the Radioactive Waste Acceptance Program (RWAP) for its conformance with WAC and potential impacts on the PA. Part of this evaluation includes a comparison of waste concentrations with the WAC action levels using a sum of fractions calculation. Waste streams with a sum of fractions greater than one or with a potential to alter PA assumptions or conceptual models require a special analysis.

Special analyses for new or revised waste streams are performed by adding the additional inventory to the Area 5 RWMS PA model and determining if all performance objectives can be met. Occasionally, waste streams may present issues other than inventory changes that require a special analysis. If the special analysis shows that all performance objectives can be met, the waste stream is recommended for approval.

Five special analyses were performed for new or revised waste streams in FY 2012 (Table 6). Two special analyses were required to evaluate a waste stream's radon-222 $\left({ }^{222} \mathrm{Rn}\right)$ flux density. The two waste streams impacting the ${ }^{222} \mathrm{Rn}$ flux density had high concentrations of radium-226 $\left({ }^{226} \mathrm{Ra}\right)$. One waste stream's cobalt-60 $\left({ }^{60} \mathrm{Co}\right)$ activity concentration exceeded WAC action levels. 
The results of these special analyses indicated that all performance objectives could be met with the addition of the waste streams to the site inventory.

Table 6. Waste Streams Evaluated by Special Analysis in FY 2012

\begin{tabular}{|c|c|c|c|}
\hline Waste Stream & Description & Issues & Result \\
\hline $\begin{array}{c}\text { ANLERESIDUES1, } \\
\text { Rev. 4 }\end{array}$ & $\begin{array}{c}\text { Argonne National Laboratory } \\
\text { Low-Level Waste Residues }\end{array}$ & $\begin{array}{c}{ }^{222} \text { Rn Flux } \\
\text { Generation }\end{array}$ & Accepted \\
\hline $\begin{array}{c}\text { DRTK000000031, } \\
\text { Rev. 0 }\end{array}$ & $\begin{array}{c}\text { Oak Ridge Radioisotope } \\
\text { Thermoelectric Generators (RTGs) }\end{array}$ & $\begin{array}{c}{ }^{90} \text { Sr Inventory, } \\
\text { Heat Generation }\end{array}$ & $\begin{array}{c}\text { Accepted with } \\
\text { Conditions after } \\
\text { Removal of } \\
\text { BUP-500 RTG }\end{array}$ \\
\hline $\begin{array}{c}\text { DRTK000000039, } \\
\text { Rev. 0 }\end{array}$ & Oak Ridge Mixed Waste RTGs \\
\hline $\begin{array}{c}\text { DRTK000000047, } \\
\text { Rev. 0 }\end{array}$ & $\begin{array}{c}\text { Lawrence Berkley National } \\
\text { Laboratory }{ }^{60} \text { Co Irradiator }\end{array}$ & $\begin{array}{c}\text { Accepted with } \\
\text { Conditions }\end{array}$ \\
\hline $\begin{array}{c}\text { DRTK000000049, } \text { Co Inventory } \\
\text { Rev. 0 }\end{array}$ & $\begin{array}{c}\text { Argonne National Laboratory } \\
\text { Sealed Sources }\end{array}$ & $\begin{array}{c}{ }^{222} \text { Rn Flux } \\
\text { Generation }\end{array}$ & Accepted \\
\hline
\end{tabular}

Two waste streams, consisting of strontium-90 $\left({ }^{90} \mathrm{Sr}\right)$ radioisotope thermoelectric generators (RTGs), required special analyses due to their ${ }^{90} \mathrm{Sr}$ inventory and heat generation. The DRTK000000031 waste stream included a 500-watt RTG produced by the Byproduct Utilization Program (BUP) known as the BUP-500. The BUP-500 contains more than 22,000 terabecquerels (TBq) (> 590,000 curies [Ci]) of ${ }^{90} \mathrm{Sr}$ and is the highest activity ${ }^{90} \mathrm{Sr}$ RTG ever constructed. Thermal analysis indicates that the heat generated by the buried BUP-500 RTG is sufficient to melt important RTG components and initiate features, events, processes (FEPs) not addressed in the PA. The DRTK0000000031 waste stream was accepted with removal of the BUP-500 and conditions on the depth and spacing of the remaining RTGs. Depth and spacing conditions are specified to manage RTG heat generation after disposal. The DRTK000000039 waste stream consisted of two low-activity RTGs classified as mixed waste due to the presence of mercury. Mixed waste must be disposed in Pit 18, a RCRA-licensed disposal unit with a high density polyethylene liner. Heat effects on the high density polyethylene liner were an additional concern for this waste stream. Thermal analysis indicates that the activity of the DRTK000000039 RTGs will not create sufficient heat to damage or significantly shorten the lifetime of the Pit 18 liner. The DRTK000000039 waste stream was accepted for disposal with conditions on the burial depth and spacing.

\subsubsection{FY 2012 Closure Inventory Estimate for the Area 3 RWMS}

The Area 3 RWMS was placed in inactive status July 1, 2006, by closing active disposal units with operational covers and suspending waste disposal operations. Although the site remains available for future disposal of large volume bulk waste streams, no waste streams are currently designated for the Area 3 RWMS. The current inventory estimate assumes no future waste disposals.

The FY 2012 inventory is unchanged from the FY 2011 inventory, which was estimated with the Area 3 Inventory model, version 2.016. The model sums past disposals and inventory revisions 
probabilistically. Probability distributions representing uncertainty in annual activity disposed are sampled each FY during operations. Radioactive decay and ingrowth during the operational period are explicitly included in the model. The estimated closure inventories are well fit by a lognormal distribution and described by the geometric mean and standard deviation estimated by the sample moments (Table 7). The estimated inventories are decayed until the assumed date of closure on October 1, 2025.

Table 7. FY 2012 Estimate of the Area 3 RWMS Inventory Disposed before September 26, 1988 (Estimates are calculated from 500 Latin hypercube sampling [LHS] realizations and decayed to October 1, 2025)

\begin{tabular}{|c|c|c|c|c|}
\hline \multirow[b]{2}{*}{ Nuclide } & \multicolumn{2}{|c|}{ U-3ax/bl } & \multicolumn{2}{|c|}{ U-3ah/at } \\
\hline & $\begin{array}{c}\text { Geometric Mean } \\
(\mathrm{Bq})\end{array}$ & $\begin{array}{c}\text { Geometric } \\
\text { Standard Deviation }\end{array}$ & $\begin{array}{c}\text { Geometric Mean } \\
(\mathrm{Bq})\end{array}$ & $\begin{array}{c}\text { Geometric Standard } \\
\text { Deviation }\end{array}$ \\
\hline $\mathrm{H}-3$ & $1.3 \mathrm{E}+14$ & 3.13 & 7.7E+11 & 2.17 \\
\hline C-14 & $1.0 \mathrm{E}+11$ & 3.13 & $1.1 \mathrm{E}+08$ & 2.88 \\
\hline Al-26 & $4.0 \mathrm{E}+06$ & 3.16 & $4.3 E+03$ & 2.90 \\
\hline $\mathrm{Cl}-36$ & $2.2 E+10$ & 3.27 & $2.4 \mathrm{E}+07$ & 2.91 \\
\hline Ar-39 & $1.0 \mathrm{E}+11$ & 3.16 & $1.1 \mathrm{E}+08$ & 2.98 \\
\hline $\mathrm{K}-40$ & $6.0 \mathrm{E}+09$ & 3.07 & $6.7 E+06$ & 2.65 \\
\hline Ca-41 & $1.6 \mathrm{E}+11$ & 3.07 & $1.7 E+08$ & 3.08 \\
\hline Co-60 & $1.2 \mathrm{E}+10$ & 3.20 & Negligible & -- \\
\hline $\mathrm{Ni}-59$ & $4.2 E+09$ & 3.13 & $4.5 E+06$ & 2.83 \\
\hline $\mathrm{Ni}-63$ & $3.4 \mathrm{E}+11$ & 3.19 & $4.0 \mathrm{E}+08$ & 2.85 \\
\hline $\mathrm{Kr}-85$ & $6.4 \mathrm{E}+10$ & 3.10 & $1.3 \mathrm{E}+08$ & 2.67 \\
\hline Sr-90 & $5.2 \mathrm{E}+12$ & 3.08 & $7.8 \mathrm{E}+09$ & 2.53 \\
\hline Zr-93 & $5.7 \mathrm{E}+08$ & 3.08 & $6.3 E+05$ & 2.67 \\
\hline $\mathrm{Nb}-93 \mathrm{~m}$ & $7.4 \mathrm{E}+10$ & 3.31 & $1.2 \mathrm{E}+08$ & 2.91 \\
\hline $\mathrm{Nb}-94$ & $1.4 \mathrm{E}+11$ & 3.26 & $1.5 \mathrm{E}+08$ & 3.01 \\
\hline Tc-99 & $1.4 \mathrm{E}+10$ & 2.45 & $1.0 \mathrm{E}+10$ & 3.81 \\
\hline Pd-107 & $2.5 E+07$ & 3.08 & $2.8 \mathrm{E}+04$ & 2.68 \\
\hline Cd-113m & $6.4 \mathrm{E}+10$ & 3.17 & $1.1 \mathrm{E}+08$ & 2.94 \\
\hline Sn-121m & $1.4 \mathrm{E}+12$ & 3.18 & $1.7 \mathrm{E}+09$ & 2.93 \\
\hline Sn-126 & $2.5 E+08$ & 3.08 & $2.7 E+05$ & 2.66 \\
\hline $\mathrm{I}-129$ & $1.3 \mathrm{E}+07$ & 3.08 & $1.4 \mathrm{E}+04$ & 2.66 \\
\hline Cs-135 & $4.4 \mathrm{E}+08$ & 3.07 & $4.9 \mathrm{E}+05$ & 2.66 \\
\hline Cs-137 & $7.2 \mathrm{E}+12$ & 3.06 & $1.0 \mathrm{E}+10$ & 2.61 \\
\hline Sm-151 & $5.5 E+11$ & 3.07 & $6.5 \mathrm{E}+08$ & 2.66 \\
\hline Eu-150 & $2.0 \mathrm{E}+11$ & 3.38 & $2.3 \mathrm{E}+08$ & 3.59 \\
\hline Eu-152 & $4.9 E+11$ & 3.25 & $8.8 \mathrm{E}+08$ & 3.02 \\
\hline Eu-154 & $8.8 \mathrm{E}+10$ & 3.26 & $2.0 \mathrm{E}+08$ & 3.17 \\
\hline Ho-166m & $5.4 \mathrm{E}+09$ & 3.17 & $5.9 E+06$ & 2.92 \\
\hline $\mathrm{Pb}-210$ & $4.0 \mathrm{E}+11$ & 4.07 & $1.1 E+05$ & 2.19 \\
\hline
\end{tabular}




\begin{tabular}{|c|c|c|c|c|}
\hline \multirow[b]{2}{*}{ Nuclide } & \multicolumn{2}{|c|}{ U-3ax/bl } & \multicolumn{2}{|c|}{ U-3ah/at } \\
\hline & $\begin{array}{c}\text { Geometric Mean } \\
(\mathrm{Bq})\end{array}$ & $\begin{array}{c}\text { Geometric } \\
\text { Standard Deviation }\end{array}$ & $\begin{array}{c}\text { Geometric Mean } \\
(\mathrm{Bq})\end{array}$ & $\begin{array}{c}\text { Geometric Standard } \\
\text { Deviation }\end{array}$ \\
\hline $\mathrm{Ra}-226$ & $5.5 \mathrm{E}+11$ & 4.07 & $3.6 \mathrm{E}+05$ & 2.19 \\
\hline Ra-228 & $1.4 \mathrm{E}+09$ & 2.71 & $4.8 E+05$ & 2.66 \\
\hline Ac-227 & $1.3 \mathrm{E}+06$ & 2.20 & $1.7 \mathrm{E}+06$ & 2.22 \\
\hline Th-228 & $8.3 E+09$ & 2.85 & $7.8 \mathrm{E}+06$ & 2.87 \\
\hline Th-229 & $1.5 \mathrm{E}+07$ & 3.05 & $1.4 \mathrm{E}+04$ & 2.62 \\
\hline Th-230 & $3.6 \mathrm{E}+07$ & 2.04 & $4.4 \mathrm{E}+07$ & 2.19 \\
\hline Th-232 & $1.5 \mathrm{E}+09$ & 2.71 & $4.9 E+05$ & 2.66 \\
\hline $\mathrm{Pa}-231$ & $3.0 \mathrm{E}+06$ & 2.21 & $4.2 \mathrm{E}+06$ & 2.22 \\
\hline $\mathrm{U}-232$ & $5.9 \mathrm{E}+09$ & 3.24 & $7.0 \mathrm{E}+06$ & 2.91 \\
\hline $\mathrm{U}-233$ & $3.5 \mathrm{E}+09$ & 3.07 & $3.9 E+06$ & 2.60 \\
\hline U-234 & $9.3 \mathrm{E}+10$ & 2.13 & $1.3 \mathrm{E}+11$ & 2.19 \\
\hline U-235 & $3.6 \mathrm{E}+09$ & 2.22 & $5.3 E+09$ & 2.22 \\
\hline U-236 & $2.5 \mathrm{E}+09$ & 2.82 & $2.4 \mathrm{E}+09$ & 2.84 \\
\hline $\mathrm{U}-238$ & $4.3 E+10$ & 2.31 & $1.1 \mathrm{E}+11$ & 2.55 \\
\hline Np-237 & $5.3 E+08$ & 2.46 & $2.3 E+08$ & 2.40 \\
\hline Pu-238 & $2.0 \mathrm{E}+11$ & 3.08 & $1.8 \mathrm{E}+10$ & 2.61 \\
\hline Pu-239 & $1.2 \mathrm{E}+12$ & 3.05 & $2.3 \mathrm{E}+09$ & 2.17 \\
\hline Pu-240 & $3.1 \mathrm{E}+11$ & 3.05 & $5.8 \mathrm{E}+08$ & 2.11 \\
\hline Pu-241 & $4.6 \mathrm{E}+11$ & 3.09 & $1.6 \mathrm{E}+09$ & 2.02 \\
\hline Pu-242 & $1.2 \mathrm{E}+08$ & 3.07 & $1.6 \mathrm{E}+05$ & 2.31 \\
\hline Am-241 & $3.8 \mathrm{E}+11$ & 3.03 & $7.0 \mathrm{E}+08$ & 2.07 \\
\hline Am-243 & $5.2 E+07$ & 3.12 & $5.7 \mathrm{E}+04$ & 2.69 \\
\hline $\mathrm{Cm}-244$ & $9.2 E+09$ & 3.10 & $1.5 \mathrm{E}+07$ & 2.66 \\
\hline Total & $1.5 E+14$ & & $1.1 E+12$ & \\
\hline
\end{tabular}

Negligible - Inventory less than 37 becquerels (Bq)

Pre-1988 waste is disposed in U-3ax/bl and U-3ah/at, with $80 \%$ of the volume and $99 \%$ of the activity disposed in U-3ax/bl. The total pre-1988 inventory as of October 1, 2025, consists of approximately $1.5 \times 10^{2} \mathrm{TBq}\left(4.1 \times 10^{3} \mathrm{Ci}\right)$ in $2.3 \times 10^{5}$ cubic meters $\left(\mathrm{m}^{3}\right)\left(8.1 \times 10^{6}\right.$ cubic feet $\left[\mathrm{ft}^{3}\right]$ ) of waste.

The post-1988 waste is disposed in U-3ah/at and U-3bh. The post-1988 inventory is estimated to consist of approximately $1.2 \times 10^{3} \mathrm{TBq}\left(3.4 \times 10^{4} \mathrm{Ci}\right)$ in $3.3 \times 10^{5} \mathrm{~m}^{3}\left(1.2 \times 10^{7} \mathrm{ft}^{3}\right)$ of waste. On an activity basis, the inventory is predominantly tritium $\left({ }^{3} \mathrm{H}\right)$ (Table 8). 
Table 8. FY 2012 Estimate of the Area 3 RWMS Inventory Disposed after September 26, 1988 (Estimates are calculated from 500 LHS realizations and decayed to October 1, 2025)

\begin{tabular}{|c|c|c|c|c|}
\hline \multirow[b]{2}{*}{ Nuclide } & \multicolumn{2}{|c|}{ U-3ah/at } & \multicolumn{2}{|c|}{ U-3bh } \\
\hline & $\begin{array}{c}\text { Geometric Mean } \\
(\mathrm{Bq})\end{array}$ & $\begin{array}{c}\text { Geometric } \\
\text { Standard Deviation }\end{array}$ & $\begin{array}{c}\text { Geometric Mean } \\
(\mathrm{Bq})\end{array}$ & $\begin{array}{c}\text { Geometric Standard } \\
\text { Deviation }\end{array}$ \\
\hline $\mathrm{H}-3$ & $7.5 \mathrm{E}+15$ & 2.06 & $4.5 \mathrm{E}+15$ & 2.15 \\
\hline C-14 & $9.8 \mathrm{E}+10$ & 1.76 & $3.0 \mathrm{E}+07$ & 2.11 \\
\hline $\mathrm{Al}-26$ & $9.5 \mathrm{E}+04$ & 2.40 & Negligible & -- \\
\hline $\mathrm{Cl}-36$ & $6.1 \mathrm{E}+08$ & 2.29 & Negligible & -- \\
\hline Ar-39 & $2.6 \mathrm{E}+09$ & 2.50 & Negligible & -- \\
\hline Ar-42 & $4.4 \mathrm{E}+08$ & 2.01 & $2.4 \mathrm{E}+08$ & 2.49 \\
\hline $\mathrm{K}-40$ & $2.6 \mathrm{E}+09$ & 1.82 & $7.1 \mathrm{E}+08$ & 2.58 \\
\hline Ca-41 & $4.0 \mathrm{E}+09$ & 2.39 & Negligible & -- \\
\hline Ti-44 & $1.2 \mathrm{E}+10$ & 2.04 & $5.6 \mathrm{E}+09$ & 2.61 \\
\hline Co-60 & $3.6 \mathrm{E}+09$ & 1.79 & $2.4 \mathrm{E}+09$ & 1.89 \\
\hline $\mathrm{Ni}-59$ & $9.4 \mathrm{E}+08$ & 2.31 & $1.7 \mathrm{E}+08$ & 2.06 \\
\hline $\mathrm{Ni}-63$ & $2.1 \mathrm{E}+11$ & 1.77 & $7.5 \mathrm{E}+09$ & 1.97 \\
\hline Se-79 & $2.5 \mathrm{E}+07$ & 2.13 & Negligible & -- \\
\hline $\mathrm{Kr}-85$ & $3.6 \mathrm{E}+09$ & 2.13 & Negligible & -- \\
\hline Sr-90 & $3.1 \mathrm{E}+14$ & 2.75 & $4.4 \mathrm{E}+10$ & 1.94 \\
\hline Zr-93 & $1.4 \mathrm{E}+07$ & 2.28 & Negligible & -- \\
\hline $\mathrm{Nb}-93 \mathrm{~m}$ & $2.8 \mathrm{E}+09$ & 2.42 & Negligible & -- \\
\hline $\mathrm{Nb}-94$ & $3.4 \mathrm{E}+09$ & 2.56 & $1.8 \mathrm{E}+08$ & 2.10 \\
\hline Tc-99 & $2.0 \mathrm{E}+12$ & 1.90 & 7.7E+10 & 1.98 \\
\hline $\mathrm{Pd}-107$ & $6.2 \mathrm{E}+05$ & 2.28 & Negligible & -- \\
\hline Cd-113m & $2.7 \mathrm{E}+09$ & 2.41 & Negligible & -- \\
\hline Sn-121m & $3.7 \mathrm{E}+10$ & 2.42 & Negligible & -- \\
\hline Sn-126 & $5.8 \mathrm{E}+08$ & 2.15 & $9.1 \mathrm{E}+05$ & 2.66 \\
\hline $\mathrm{I}-129$ & $4.7 \mathrm{E}+08$ & 2.03 & $2.4 \mathrm{E}+08$ & 2.63 \\
\hline Cs-135 & $1.1 \mathrm{E}+07$ & 2.29 & Negligible & -- \\
\hline Cs-137 & $1.7 \mathrm{E}+14$ & 1.96 & $4.9 \mathrm{E}+10$ & 1.75 \\
\hline Ba-133 & $5.0 \mathrm{E}+09$ & 1.99 & $1.6 \mathrm{E}+09$ & 2.73 \\
\hline Sm-151 & $1.5 \mathrm{E}+10$ & 2.28 & $1.2 \mathrm{E}+06$ & 2.23 \\
\hline Eu-150 & $6.1 E+09$ & 2.76 & Negligible & -- \\
\hline Eu-152 & $3.9 \mathrm{E}+10$ & 1.87 & $1.3 \mathrm{E}+09$ & 2.42 \\
\hline Eu-154 & $8.6 \mathrm{E}+09$ & 1.99 & $1.6 \mathrm{E}+08$ & 2.04 \\
\hline Ho-166m & $1.3 \mathrm{E}+08$ & 2.38 & Negligible & -- \\
\hline $\mathrm{Pb}-210$ & $9.6 \mathrm{E}+10$ & 1.77 & $4.5 E+08$ & 1.86 \\
\hline $\mathrm{Bi}-207$ & $3.8 \mathrm{E}+05$ & 2.27 & $1.8 \mathrm{E}+07$ & 2.19 \\
\hline Bi-210m & $6.7 \mathrm{E}+06$ & 1.96 & $2.1 \mathrm{E}+08$ & 2.23 \\
\hline $\mathrm{Ra}-226$ & $1.0 \mathrm{E}+11$ & 1.98 & $9.4 \mathrm{E}+08$ & 2.25 \\
\hline
\end{tabular}




\begin{tabular}{|c|c|c|c|c|}
\hline \multirow[b]{2}{*}{ Nuclide } & \multicolumn{2}{|c|}{ U-3ah/at } & \multicolumn{2}{|c|}{ U-3bh } \\
\hline & $\begin{array}{l}\text { Geometric Mean } \\
\text { (Bq) }\end{array}$ & $\begin{array}{c}\text { Geometric } \\
\text { Standard Deviation }\end{array}$ & $\begin{array}{c}\text { Geometric Mean } \\
(\mathrm{Bq})\end{array}$ & $\begin{array}{c}\text { Geometric Standard } \\
\text { Deviation }\end{array}$ \\
\hline Ra-228 & 1.3E+10 & 1.69 & $1.9 \mathrm{E}+11$ & 2.70 \\
\hline Ac-227 & $2.5 \mathrm{E}+09$ & 1.85 & $1.4 \mathrm{E}+06$ & 2.15 \\
\hline Th-228 & $7.2 \mathrm{E}+10$ & 1.91 & $1.8 \mathrm{E}+11$ & 2.70 \\
\hline Th-229 & $4.0 \mathrm{E}+07$ & 1.95 & $4.8 \mathrm{E}+07$ & 2.53 \\
\hline Th-230 & $4.7 \mathrm{E}+10$ & 2.00 & $7.1 \mathrm{E}+10$ & 2.72 \\
\hline Th-232 & $1.4 \mathrm{E}+10$ & 1.71 & $2.0 \mathrm{E}+11$ & 2.70 \\
\hline Pa-231 & $3.8 \mathrm{E}+08$ & 1.79 & $5.0 \mathrm{E}+06$ & 2.16 \\
\hline $\mathrm{U}-232$ & $5.3 \mathrm{E}+10$ & 2.20 & Negligible & -- \\
\hline$U-233$ & $1.6 \mathrm{E}+10$ & 1.93 & $2.2 \mathrm{E}+10$ & 2.52 \\
\hline U-234 & $7.4 \mathrm{E}+12$ & 1.98 & $1.3 \mathrm{E}+11$ & 2.08 \\
\hline U-235 & $3.4 \mathrm{E}+11$ & 1.83 & $1.1 \mathrm{E}+10$ & 2.18 \\
\hline U-236 & $3.6 \mathrm{E}+11$ & 2.34 & $9.6 \mathrm{E}+07$ & 2.71 \\
\hline $\mathrm{U}-238$ & $1.3 \mathrm{E}+13$ & 1.74 & $5.8 \mathrm{E}+11$ & 2.32 \\
\hline Np-237 & $2.4 \mathrm{E}+11$ & 2.08 & $1.5 \mathrm{E}+08$ & 1.91 \\
\hline Pu-238 & $5.6 \mathrm{E}+11$ & 1.97 & $1.8 \mathrm{E}+11$ & 2.07 \\
\hline Pu-239 & $2.7 \mathrm{E}+12$ & 1.68 & $5.1 \mathrm{E}+11$ & 1.85 \\
\hline Pu-240 & $5.4 \mathrm{E}+11$ & 1.70 & $8.6 \mathrm{E}+10$ & 2.07 \\
\hline Pu-241 & $1.5 \mathrm{E}+12$ & 1.75 & $1.6 \mathrm{E}+11$ & 2.00 \\
\hline Pu-242 & $1.1 \mathrm{E}+08$ & 1.61 & $4.0 \mathrm{E}+07$ & 2.32 \\
\hline Am-241 & $5.3 \mathrm{E}+11$ & 1.56 & $8.8 \mathrm{E}+10$ & 1.84 \\
\hline Am-242m & $2.3 \mathrm{E}+08$ & 2.18 & $3.3 \mathrm{E}+06$ & 2.84 \\
\hline Am-243 & $5.9 \mathrm{E}+08$ & 1.80 & $4.3 \mathrm{E}+07$ & 2.63 \\
\hline $\mathrm{Cm}-243$ & $3.1 \mathrm{E}+06$ & 1.74 & $9.9 E+05$ & 2.61 \\
\hline $\mathrm{Cm}-244$ & $8.2 \mathrm{E}+09$ & 1.60 & $1.1 E+08$ & 2.09 \\
\hline $\mathrm{Cm}-245$ & $5.4 \mathrm{E}+08$ & 1.90 & $8.2 E+06$ & 2.64 \\
\hline $\mathrm{Cm}-246$ & $8.8 \mathrm{E}+07$ & 1.86 & Negligible & -- \\
\hline $\mathrm{Cm}-247$ & $7.0 \mathrm{E}+05$ & 2.72 & Negligible & -- \\
\hline Cf-249 & $3.4 \mathrm{E}+03$ & 2.21 & Negligible & -- \\
\hline Cf-250 & $1.3 \mathrm{E}+03$ & 2.81 & Negligible & -- \\
\hline Cf-251 & $2.2 \mathrm{E}+08$ & 2.29 & Negligible & -- \\
\hline Total & 8.0E+15 & & $4.5 E+15$ & \\
\hline
\end{tabular}

Negligible - Inventory less than $37 \mathrm{~Bq}$

The volume of waste disposed at the Area 3 RWMS is divided approximately equally between the pre- and post-1988 period (Figure 1). The total activity was disposed predominately in the post-1988 period since 2000 (Figure 2). 


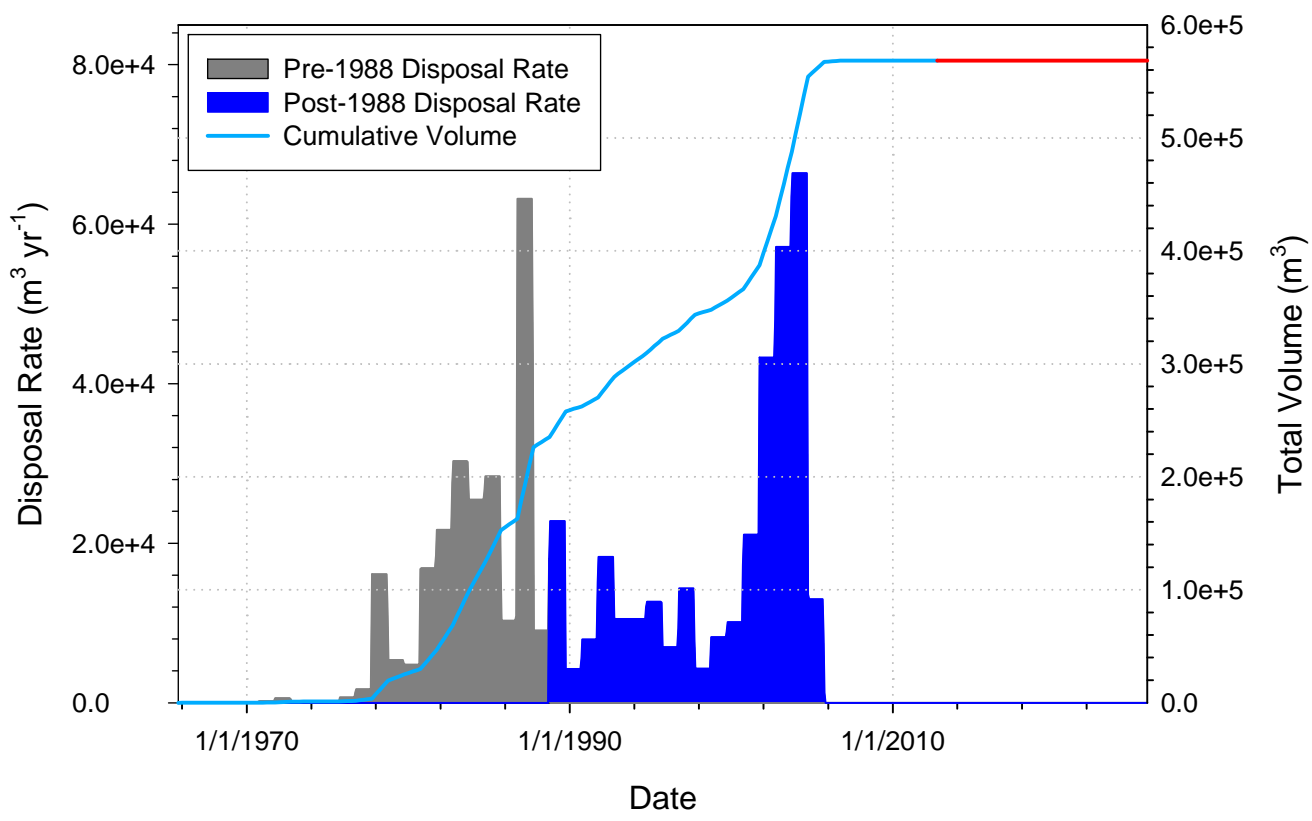

Figure 1. Annual Volume Disposal Rate and Total Volume for the Area 3 RWMS

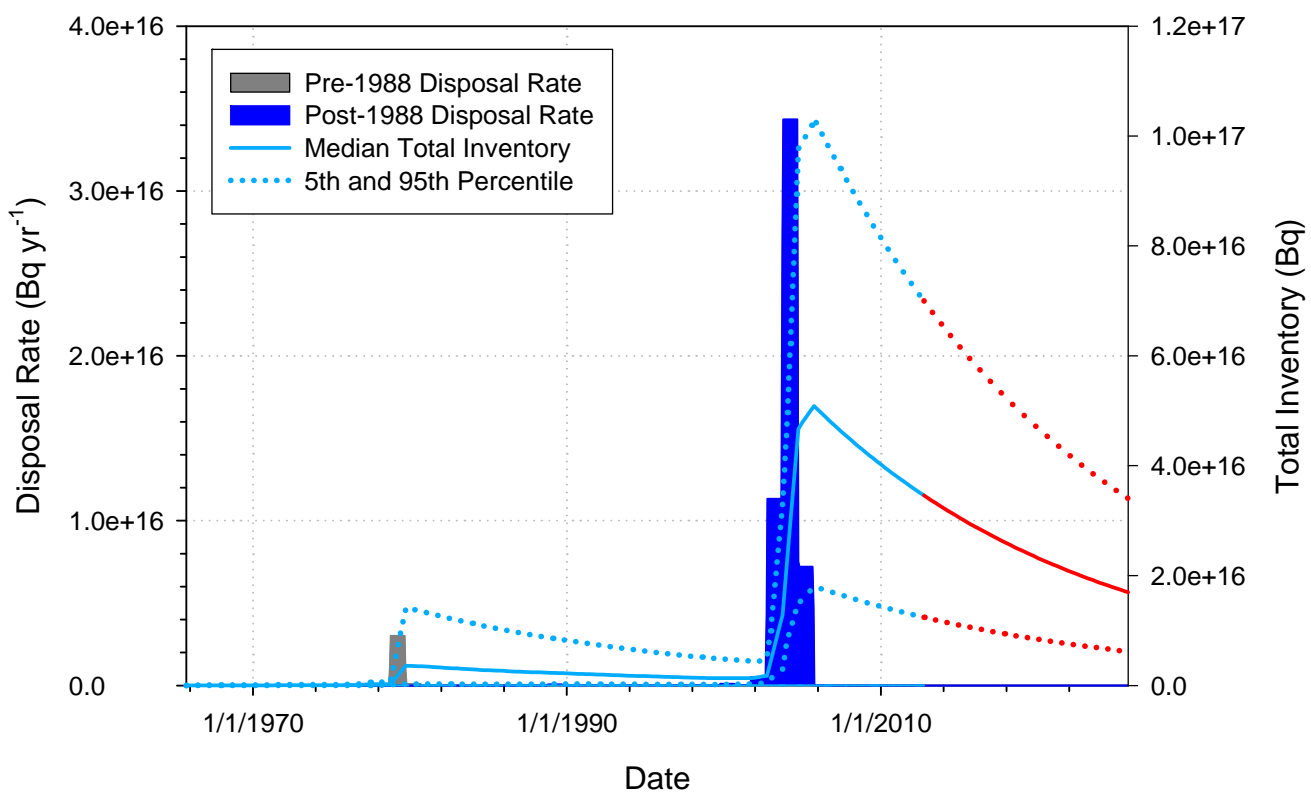

Figure 2. Activity Annual Disposal Rate and Total Inventory for the Area 3 RWMS

\subsubsection{FY 2012 Closure Inventory Estimate for the Area 5 RWMS}

The Area 5 RWMS PA GoldSim model divides the site inventory into three virtual disposal units based on the depth of burial. Most wastes are disposed in shallow land burial (SLB) disposal units. Wastes capable of producing significant ${ }^{222} \mathrm{Rn}$ flux densities are disposed below thicker covers in two radium disposal units (RaDUs), the lower cell of Pit 6 and the northern section of 
Pit 13. High specific activity and TRU wastes were disposed in GCD boreholes. The inventory of the three virtual disposal units is further divided into pre-1988, post-1988 disposed, and future portions.

The FY 2012 estimate of the Area 5 RWMS closure inventory was prepared using the GoldSim Area 5 Inventory v2.110 model. No significant changes were made to the Area 5 inventory model in FY 2012, except addition of the FY 2012 disposed waste. The model sums past disposals, revisions, and future inventory estimates probabilistically. Probability distributions representing uncertainty in annual activity disposed are sampled each FY during operations. Radioactive decay and ingrowth during the operational period are explicitly included in the model. The closure inventory estimates are well fit by a lognormal distribution and described by the geometric mean and standard deviation estimated as the sample moments (Table 9). The estimated inventories are decayed until the assumed date of closure on October 1, 2028.

Table 9. FY 2012 Estimate of the Area 5 RWMS SLB Inventory (Estimates are calculated from 500 LHS realizations and decayed to October 1,2028 )

\begin{tabular}{|c|c|c|c|c|c|c|}
\hline \multirow[b]{2}{*}{ Nuclide } & \multicolumn{2}{|c|}{ Pre-1988 SLB } & \multicolumn{2}{|c|}{ Post-1988 SLB } & \multicolumn{2}{|c|}{ Future SLB } \\
\hline & $\begin{array}{l}\text { Geometric } \\
\text { Mean }(\mathrm{Bq})\end{array}$ & $\begin{array}{l}\text { Geometric } \\
\text { Standard } \\
\text { Deviation }\end{array}$ & $\begin{array}{l}\text { Geometric } \\
\text { Mean }(\mathrm{Bq})\end{array}$ & $\begin{array}{l}\text { Geometric } \\
\text { Standard } \\
\text { Deviation }\end{array}$ & $\begin{array}{l}\text { Geometric } \\
\text { Mean }(\mathrm{Bq})\end{array}$ & $\begin{array}{l}\text { Geometric } \\
\text { Standard } \\
\text { Deviation }\end{array}$ \\
\hline $\mathrm{H}-3$ & $3.4 \mathrm{E}+16$ & 1.84 & $3.4 \mathrm{E}+16$ & 1.52 & $5.6 \mathrm{E}+16$ & 2.67 \\
\hline C-14 & $2.9 \mathrm{E}+11$ & 1.81 & $2.6 E+13$ & 2.06 & 1.7E+04 & 42.69 \\
\hline Al-26 & $9.1 \mathrm{E}+06$ & 1.93 & $6.7 \mathrm{E}+05$ & 1.88 & $6.2 \mathrm{E}+06$ & 5.34 \\
\hline $\mathrm{Cl}-36$ & $5.1 \mathrm{E}+10$ & 1.92 & $2.4 \mathrm{E}+08$ & 2.18 & $0.0 \mathrm{E}+00$ & 1.01 \\
\hline Ar-39 & $2.3 E+11$ & 1.91 & $1.0 E+09$ & 2.36 & $1.4 \mathrm{E}+07$ & 88.67 \\
\hline Ar-42 & Negligible & -- & $6.6 \mathrm{E}+08$ & 2.01 & $9.2 \mathrm{E}+09$ & 2.27 \\
\hline $\mathrm{K}-40$ & $1.3 \mathrm{E}+10$ & 1.88 & $3.3 E+10$ & 1.52 & $1.2 \mathrm{E}+04$ & 1488.56 \\
\hline $\mathrm{Ca}-41$ & $3.8 \mathrm{E}+11$ & 1.95 & $1.5 \mathrm{E}+09$ & 2.39 & $4.9 E+08$ & 100.15 \\
\hline $\mathrm{Ti}-44$ & Negligible & -- & $2.2 \mathrm{E}+10$ & 2.03 & $3.5 E+14$ & 3.80 \\
\hline Co-60 & $2.2 \mathrm{E}+12$ & 2.40 & $3.3 E+14$ & 1.66 & $4.4 \mathrm{E}+11$ & 3.90 \\
\hline $\mathrm{Ni}-59$ & $9.6 \mathrm{E}+09$ & 1.89 & $2.8 \mathrm{E}+12$ & 1.67 & $4.7 E+13$ & 3.49 \\
\hline $\mathrm{Ni}-63$ & $7.3 \mathrm{E}+11$ & 1.91 & $2.6 \mathrm{E}+14$ & 1.67 & $1.6 \mathrm{E}+11$ & 67.22 \\
\hline Se-79 & Negligible & -- & $3.8 \mathrm{E}+12$ & 1.92 & $3.3 E+09$ & 3.16 \\
\hline $\mathrm{Kr}-85$ & $4.3 \mathrm{E}+11$ & 2.50 & $9.4 \mathrm{E}+09$ & 1.62 & $3.8 \mathrm{E}+15$ & 5.92 \\
\hline Sr-90 & $1.6 \mathrm{E}+15$ & 3.87 & $1.9 \mathrm{E}+16$ & 2.03 & $1.3 E+07$ & 3.78 \\
\hline Zr-93 & $1.3 \mathrm{E}+09$ & 1.89 & $1.2 \mathrm{E}+08$ & 1.72 & $7.9 \mathrm{E}+09$ & 18.38 \\
\hline $\mathrm{Nb}-93 \mathrm{~m}$ & $1.2 \mathrm{E}+11$ & 1.93 & $1.1 \mathrm{E}+09$ & 2.21 & $1.1 \mathrm{E}+14$ & 2.72 \\
\hline $\mathrm{Nb}-94$ & $3.1 E+11$ & 1.96 & $2.1 E+11$ & 2.16 & $5.2 E+04$ & 10.49 \\
\hline Mo-93 & Negligible & -- & $3.9 \mathrm{E}+08$ & 2.75 & $4.5 E+08$ & 352.97 \\
\hline Tc-98 & Negligible & -- & Negligible & -- & $1.2 \mathrm{E}+09$ & 69.95 \\
\hline Tc-99 & $1.4 \mathrm{E}+13$ & 2.65 & $5.0 \mathrm{E}+14$ & 1.61 & $3.5 \mathrm{E}+04$ & 65.16 \\
\hline Pd-107 & $5.6 \mathrm{E}+07$ & 1.88 & $8.8 \mathrm{E}+05$ & 1.75 & $1.8 \mathrm{E}+09$ & 38.01 \\
\hline $\mathrm{Ag}-108 \mathrm{~m}$ & Negligible & -- & $2.3 E+11$ & 2.79 & $2.6 \mathrm{E}+09$ & 4.11 \\
\hline
\end{tabular}




\begin{tabular}{|c|c|c|c|c|c|c|}
\hline \multirow[b]{2}{*}{ Nuclide } & \multicolumn{2}{|c|}{ Pre-1988 SLB } & \multicolumn{2}{|c|}{ Post-1988 SLB } & \multicolumn{2}{|c|}{ Future SLB } \\
\hline & $\begin{array}{l}\text { Geometric } \\
\text { Mean }(\mathrm{Bq})\end{array}$ & $\begin{array}{l}\text { Geometric } \\
\text { Standard } \\
\text { Deviation }\end{array}$ & $\begin{array}{l}\text { Geometric } \\
\text { Mean }(\mathrm{Bq})\end{array}$ & $\begin{array}{l}\text { Geometric } \\
\text { Standard } \\
\text { Deviation }\end{array}$ & $\begin{array}{l}\text { Geometric } \\
\text { Mean }(\mathrm{Bq})\end{array}$ & $\begin{array}{l}\text { Geometric } \\
\text { Standard } \\
\text { Deviation }\end{array}$ \\
\hline Cd-113m & $1.0 \mathrm{E}+11$ & 1.94 & $3.4 \mathrm{E}+10$ & 2.19 & $9.7 E+05$ & 48.90 \\
\hline In-115 & Negligible & -- & Negligible & -- & $2.4 \mathrm{E}+14$ & 3.60 \\
\hline Sn-121m & $2.7 E+12$ & 1.94 & $1.4 \mathrm{E}+10$ & 2.42 & $9.2 E+09$ & 3.48 \\
\hline Sn-126 & $5.4 \mathrm{E}+08$ & 1.89 & $3.8 E+10$ & 1.85 & $1.2 \mathrm{E}+11$ & 34.88 \\
\hline Te-123 & Negligible & -- & $2.3 E+09$ & 2.61 & $3.5 \mathrm{E}+00$ & 10.89 \\
\hline $\mathrm{I}-129$ & $4.0 \mathrm{E}+07$ & 1.82 & $1.7 \mathrm{E}+10$ & 1.97 & $7.3 \mathrm{E}+12$ & 14.61 \\
\hline Cs-135 & $9.8 \mathrm{E}+08$ & 1.89 & $3.3 E+07$ & 1.88 & $2.2 \mathrm{E}+13$ & 11.59 \\
\hline Cs-137 & $3.7 \mathrm{E}+15$ & 3.10 & $9.9 E+14$ & 1.90 & $1.1 \mathrm{E}-01$ & 16.77 \\
\hline Ba-133 & $1.8 \mathrm{E}+08$ & 2.81 & $1.6 \mathrm{E}+10$ & 1.70 & $1.4 \mathrm{E}+04$ & 2511.13 \\
\hline La-138 & Negligible & -- & Negligible & -- & $7.2 \mathrm{E}+09$ & 1625.94 \\
\hline Ce-138 & Negligible & -- & Negligible & -- & $2.0 \mathrm{E}+11$ & 2.51 \\
\hline $\mathrm{Nd}-144$ & Negligible & -- & Negligible & -- & $2.8 \mathrm{E}+06$ & 4.44 \\
\hline Pm-145 & Negligible & -- & $1.5 \mathrm{E}+07$ & 2.72 & $2.0 \mathrm{E}+04$ & 817.18 \\
\hline Pm-146 & Negligible & -- & $2.3 \mathrm{E}+06$ & 2.42 & $3.1 \mathrm{E}+11$ & 2.97 \\
\hline Sm-146 & Negligible & -- & Negligible & -- & $3.1 \mathrm{E}+11$ & 2.97 \\
\hline Sm-147 & Negligible & -- & $3.3 \mathrm{E}+04$ & 2.73 & $2.9 \mathrm{E}+11$ & 2.30 \\
\hline Sm-148 & Negligible & -- & Negligible & -- & $1.3 \mathrm{E}+10$ & 6.93 \\
\hline Sm-151 & $1.1 \mathrm{E}+12$ & 1.88 & $2.4 \mathrm{E}+12$ & 2.55 & $7.2 \mathrm{E}+11$ & 2.32 \\
\hline Eu-150 & $4.0 \mathrm{E}+11$ & 2.07 & $2.2 \mathrm{E}+09$ & 2.68 & $6.2 \mathrm{E}+10$ & 4.41 \\
\hline Eu-152 & $2.9 \mathrm{E}+12$ & 2.22 & $5.2 \mathrm{E}+13$ & 1.97 & $1.9 \mathrm{E}+11$ & 3.30 \\
\hline Eu-154 & $3.1 \mathrm{E}+11$ & 2.12 & $7.1 \mathrm{E}+13$ & 1.73 & $4.0 \mathrm{E}+11$ & 2.42 \\
\hline Gd-148 & Negligible & -- & $1.1 \mathrm{E}+07$ & 2.62 & $2.6 \mathrm{E}+09$ & 2.03 \\
\hline Gd-152 & Negligible & -- & Negligible & -- & $3.4 \mathrm{E}+11$ & 3.61 \\
\hline Tb-157 & Negligible & -- & $1.2 \mathrm{E}+07$ & 2.65 & $9.8 \mathrm{E}+12$ & 7.31 \\
\hline Ho-166m & $1.2 \mathrm{E}+10$ & 1.89 & $2.8 \mathrm{E}+08$ & 1.92 & $5.0 \mathrm{E}+13$ & 1.76 \\
\hline Hf-182 & Negligible & -- & Negligible & -- & $2.7 \mathrm{E}+12$ & 1.65 \\
\hline Os-194 & Negligible & -- & Negligible & -- & $1.5 \mathrm{E}+12$ & 2.07 \\
\hline Ir-192m & Negligible & -- & Negligible & -- & $1.4 \mathrm{E}+14$ & 1.77 \\
\hline Pt-193 & Negligible & -- & $6.9 \mathrm{E}+11$ & 1.98 & $4.1 E+10$ & 2.36 \\
\hline $\mathrm{Pb}-210$ & $1.1 \mathrm{E}+12$ & 2.57 & $8.0 \mathrm{E}+11$ & 1.60 & $3.2 E+12$ & 1.97 \\
\hline $\mathrm{Bi}-207$ & $6.0 \mathrm{E}+05$ & 3.15 & $1.6 \mathrm{E}+07$ & 1.74 & $5.4 \mathrm{E}+12$ & 1.88 \\
\hline $\mathrm{Bi}-210 \mathrm{~m}$ & Negligible & -- & $5.8 \mathrm{E}+07$ & 2.21 & $1.7 \mathrm{E}+12$ & 2.53 \\
\hline Ra-226 & $1.5 \mathrm{E}+12$ & 2.58 & $1.1 \mathrm{E}+12$ & 1.78 & $1.7 \mathrm{E}+13$ & 2.83 \\
\hline Ra-228 & $5.0 \mathrm{E}+10$ & 2.23 & $6.9 \mathrm{E}+11$ & 1.45 & $5.7 \mathrm{E}+10$ & 13.49 \\
\hline Ac-227 & $1.2 \mathrm{E}+10$ & 1.80 & $1.1 \mathrm{E}+11$ & 2.09 & $2.5 \mathrm{E}+05$ & 8.13 \\
\hline Th-228 & $6.8 \mathrm{E}+10$ & 1.95 & $2.8 \mathrm{E}+12$ & 1.64 & $2.5 \mathrm{E}+12$ & 2.35 \\
\hline Th-229 & $1.8 \mathrm{E}+08$ & 2.10 & $6.2 \mathrm{E}+11$ & 1.92 & $3.6 E+08$ & 4.02 \\
\hline
\end{tabular}




\begin{tabular}{|c|c|c|c|c|c|c|}
\hline \multirow[b]{2}{*}{ Nuclide } & \multicolumn{2}{|c|}{ Pre-1988 SLB } & \multicolumn{2}{|c|}{ Post-1988 SLB } & \multicolumn{2}{|c|}{ Future SLB } \\
\hline & $\begin{array}{l}\text { Geometric } \\
\text { Mean }(\mathrm{Bq})\end{array}$ & $\begin{array}{l}\text { Geometric } \\
\text { Standard } \\
\text { Deviation }\end{array}$ & $\begin{array}{l}\text { Geometric } \\
\text { Mean }(\mathrm{Bq})\end{array}$ & $\begin{array}{l}\text { Geometric } \\
\text { Standard } \\
\text { Deviation }\end{array}$ & $\begin{array}{l}\text { Geometric } \\
\text { Mean }(\mathrm{Bq})\end{array}$ & $\begin{array}{l}\text { Geometric } \\
\text { Standard } \\
\text { Deviation }\end{array}$ \\
\hline Th-230 & $4.5 E+10$ & 1.80 & $3.1 E+11$ & 1.46 & $8.6 \mathrm{E}+09$ & 3.74 \\
\hline Th-232 & $5.0 \mathrm{E}+10$ & 2.23 & $7.2 \mathrm{E}+11$ & 1.44 & $3.7 \mathrm{E}+09$ & 5.18 \\
\hline $\mathrm{Pa}-231$ & $8.0 \mathrm{E}+09$ & 1.77 & $1.2 \mathrm{E}+10$ & 1.41 & $7.0 \mathrm{E}+11$ & 3.56 \\
\hline $\mathrm{U}-232$ & $1.3 E+10$ & 1.94 & $2.0 \mathrm{E}+12$ & 1.90 & $5.5 E+10$ & 10.39 \\
\hline U-233 & $3.8 E+10$ & 2.18 & $1.4 \mathrm{E}+14$ & 2.29 & $1.3 \mathrm{E}+10$ & 5.25 \\
\hline $\mathrm{U}-234$ & $9.0 \mathrm{E}+13$ & 1.99 & $1.6 \mathrm{E}+14$ & 1.37 & $6.9 \mathrm{E}+06$ & 35.51 \\
\hline $\mathrm{U}-235$ & $3.7 \mathrm{E}+12$ & 2.02 & $7.2 \mathrm{E}+12$ & 1.37 & $4.5 \mathrm{E}+09$ & 5.18 \\
\hline $\mathrm{U}-236$ & $1.2 \mathrm{E}+12$ & 2.58 & $7.3 E+12$ & 1.53 & $1.8 \mathrm{E}+08$ & 2.81 \\
\hline $\mathrm{U}-238$ & $1.0 E+14$ & 2.17 & $4.0 \mathrm{E}+14$ & 1.46 & $1.2 \mathrm{E}+05$ & 12.04 \\
\hline $\mathrm{Np}-237$ & $2.5 E+11$ & 1.96 & $2.1 \mathrm{E}+11$ & 1.51 & $7.4 \mathrm{E}+06$ & 14.08 \\
\hline Pu-238 & $6.7 E+12$ & 1.89 & $6.9 \mathrm{E}+12$ & 1.51 & $3.2 E+12$ & 1.97 \\
\hline Pu-239 & $1.4 \mathrm{E}+13$ & 1.87 & $1.7 \mathrm{E}+13$ & 1.40 & $5.4 \mathrm{E}+12$ & 1.88 \\
\hline Pu-240 & $3.5 E+12$ & 1.80 & $6.5 \mathrm{E}+12$ & 1.59 & $1.7 \mathrm{E}+12$ & 2.53 \\
\hline $\mathrm{Pu}-241$ & $3.9 E+12$ & 1.78 & $4.1 \mathrm{E}+13$ & 1.82 & $1.7 \mathrm{E}+13$ & 2.83 \\
\hline Pu-242 & $7.5 E+08$ & 1.74 & $4.7 \mathrm{E}+11$ & 2.19 & $5.7 \mathrm{E}+10$ & 13.49 \\
\hline $\mathrm{Pu}-244$ & $4.5 E+09$ & 4.08 & $2.9 \mathrm{E}+06$ & 1.85 & $2.5 \mathrm{E}+05$ & 8.13 \\
\hline Am-241 & $4.5 E+12$ & 1.66 & $1.0 \mathrm{E}+13$ & 1.49 & $2.5 E+12$ & 2.35 \\
\hline Am-242m & Negligible & -- & $1.6 \mathrm{E}+09$ & 1.77 & $3.6 \mathrm{E}+08$ & 4.02 \\
\hline Am-243 & $4.8 E+08$ & 2.36 & $5.5 \mathrm{E}+10$ & 1.78 & $8.6 E+09$ & 3.74 \\
\hline $\mathrm{Cm}-243$ & $6.1 \mathrm{E}+09$ & 2.41 & $1.9 \mathrm{E}+10$ & 2.01 & $3.7 \mathrm{E}+09$ & 5.18 \\
\hline $\mathrm{Cm}-244$ & $7.1 \mathrm{E}+10$ & 2.53 & $2.6 \mathrm{E}+12$ & 1.84 & $7.0 \mathrm{E}+11$ & 3.56 \\
\hline $\mathrm{Cm}-245$ & $1.4 \mathrm{E}+05$ & 3.30 & $5.5 \mathrm{E}+11$ & 1.95 & $5.5 E+10$ & 10.39 \\
\hline $\mathrm{Cm}-246$ & $9.2 E+04$ & 2.61 & $9.5 \mathrm{E}+10$ & 1.77 & $1.3 \mathrm{E}+10$ & 5.25 \\
\hline $\mathrm{Cm}-247$ & Negligible & -- & $1.1 \mathrm{E}+08$ & 1.86 & $6.9 \mathrm{E}+06$ & 35.51 \\
\hline $\mathrm{Cm}-248$ & $7.5 E+04$ & 3.08 & $7.7 \mathrm{E}+07$ & 1.76 & $4.5 E+09$ & 5.18 \\
\hline Cf-249 & Negligible & -- & $1.0 \mathrm{E}+09$ & 1.65 & $1.8 \mathrm{E}+08$ & 2.81 \\
\hline Cf-250 & $2.9 \mathrm{E}+05$ & 2.43 & $9.9 E+05$ & 1.81 & $1.2 \mathrm{E}+05$ & 12.04 \\
\hline Cf-251 & Negligible & -- & $9.6 \mathrm{E}+07$ & 1.88 & $7.4 \mathrm{E}+06$ & 14.08 \\
\hline Total & $4.0 \mathrm{E}+16$ & & $5.6 \mathrm{E}+16$ & & $6.1 E+16$ & \\
\hline
\end{tabular}

Negligible - Inventory less than $37 \mathrm{~Bq}$

The total Area 5 RWMS inventory estimate in FY 2012 is not significantly different from the FY 2011 estimate. Significant increases, however, are noted for two individual radionuclides, ${ }^{226} \mathrm{Ra}$ and technetium-99 ( $\left.{ }^{99} \mathrm{Tc}\right)$. Two radionuclides not previously reported, molybdenum-93 and terbium-157, were disposed in FY 2012. Previously, any radionuclide with a total site inventory exceeding $3.7 \times 10^{7} \mathrm{~Bq}$ (0.001 Ci) was included in the PA/CA model. In FY 2012, new radionuclide-specific screening limits were developed to identify radionuclides to include in the PA/CA model. The new limits confirm that the previous $3.7 \times 10^{7} \mathrm{~Bq}(0.001 \mathrm{Ci})$ limit was very 
conservative. Application of the screening limits to the disposed inventory did not identify any new radionuclides for inclusion in the PA/CA model.

The median SLB volume estimate has increased from $8.6 \times 10^{5} \mathrm{~m}^{3}\left(3.0 \times 10^{7} \mathrm{ft}^{3}\right)$ in FY 2011 to $9.1 \times 10^{5} \mathrm{~m}^{3}\left(3.2 \times 10^{7} \mathrm{ft}^{3}\right)$ in FY 2012 (Figure 3). The median post-1988 SLB volume has increased from $6.8 \times 10^{5} \mathrm{~m}^{3}\left(2.4 \times 10^{7} \mathrm{ft}^{3}\right)$ in FY 2011 to $7.3 \times 10^{5} \mathrm{~m}^{3}\left(2.6 \times 10^{7} \mathrm{ft}^{3}\right)$ in FY 2012. The increases are due to increases in the future waste forecast.

The median closure inventory estimate decreased insignificantly from $2.0 \times 10^{5} \mathrm{TBq}\left(5.4 \times 10^{6} \mathrm{Ci}\right)$ in FY 2011 to $1.9 \times 10^{5} \mathrm{TBq}\left(5.1 \times 10^{6} \mathrm{Ci}\right)$ in FY 2012 (Figure 4). The median post-1988 closure inventory estimate increased slightly from $1.1 \times 10^{5} \mathrm{TBq}\left(3.1 \times 10^{6} \mathrm{Ci}\right)$ in FY 2011 to $1.2 \times 10^{5} \mathrm{TBq}$ $\left(3.2 \times 10^{6} \mathrm{Ci}\right)$ in FY 2012 .

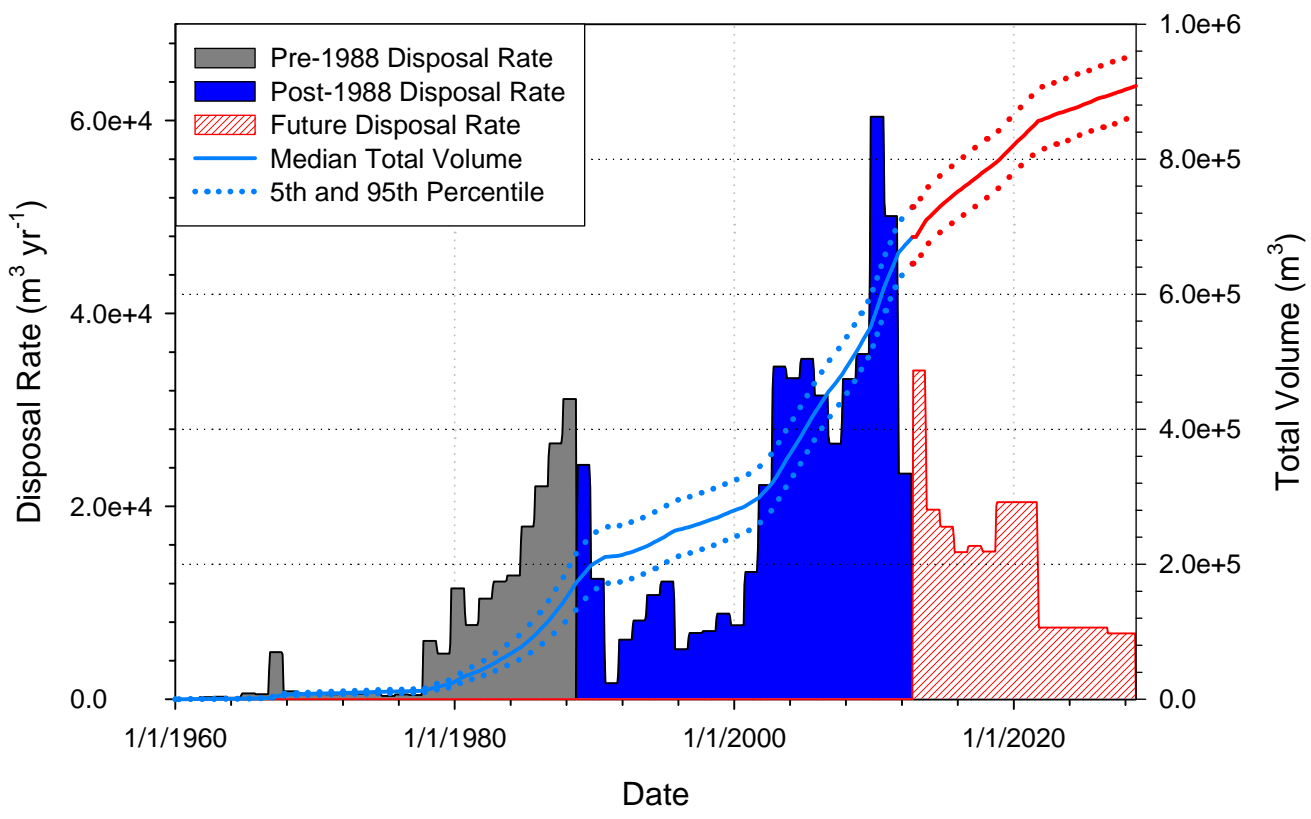

Figure 3. Annual Volume Disposal Rate and Total Volume for the Area 5 RWMS SLB Disposal Units 


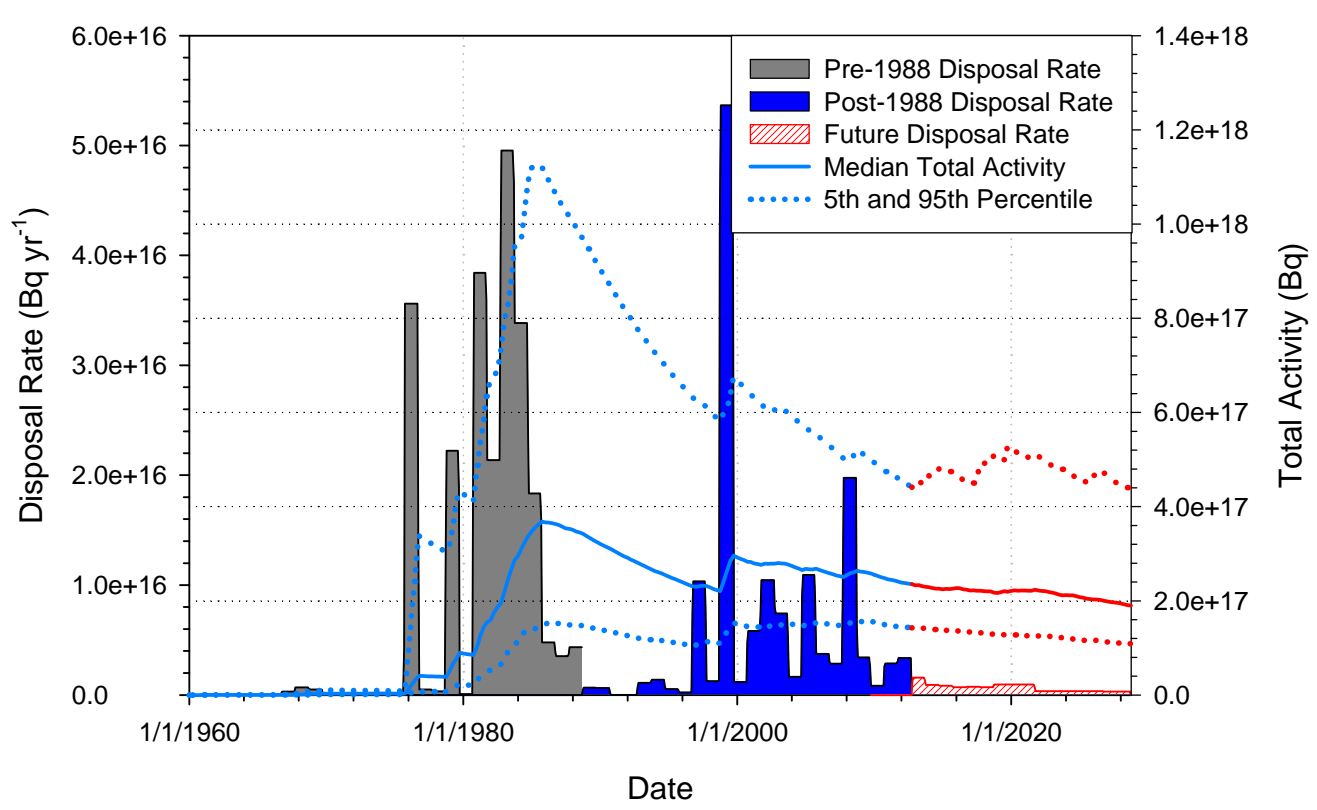

Figure 4. Annual Activity Disposal Rate and Total Inventory for the Area 5 RWMS SLB Disposal Units

\section{RaDU Inventory}

The lower cell of Pit 6 and Pit 13 were excavated to greater depth to contain thorium wastes that have the potential to generate ${ }^{222} \mathrm{Rn}$ in the future, as ${ }^{226} \mathrm{Ra}$ is produced by the decay of thorium-230 $\left({ }^{230} \mathrm{Th}\right)$. The inventory of both disposal units is predominately ${ }^{232} \mathrm{Th}$. The lower cell of Pit 6 was operated from FY 1992 to FY 2002. The Pit 6 lower cell inventory remains unchanged from previous years (Table 10). The upper cell of Pit 6 was filled and closed in FY 2011.

Pit 13 began operations in FY 2004 with disposal of the Defense National Stockpile Center thorium nitrate waste stream. The entire thorium nitrate waste stream was disposed in FY 2004 and 2005 in a single layer, with the top of the waste 6.4 meters (m) (21 feet [ft]) below grade. In FY 2008 for PA modeling purposes, Pit 13 was divided into a northern RaDU portion containing the thorium nitrate waste below a thicker cover and a southern SLB portion with LLW below a thinner cover. The Pit $13 \mathrm{RaDU}$ inventory is summarized in Table 10. The Pit 13 SLB inventory is included in the post-1988 SLB inventory. 
Table 10. FY 2012 Estimate of the Area 5 RWMS RaDU Inventory Disposed (Estimates are calculated from 500 LHS realizations and decayed to October 1,2028 )

\begin{tabular}{|c|c|c|c|c|c|c|}
\hline \multirow[b]{2}{*}{ Nuclide } & \multicolumn{2}{|c|}{ Pit 6 (Upper Cell) } & \multicolumn{2}{|c|}{ Pit 6 (Lower Cell) RaDU } & \multicolumn{2}{|c|}{ Pit 13 RaDU } \\
\hline & $\begin{array}{l}\text { Geometric } \\
\text { Mean }(\mathrm{Bq})\end{array}$ & $\begin{array}{l}\text { Geometric } \\
\text { Standard } \\
\text { Deviation }\end{array}$ & $\begin{array}{l}\text { Geometric } \\
\text { Mean (Bq) }\end{array}$ & $\begin{array}{l}\text { Geometric } \\
\text { Standard } \\
\text { Deviation }\end{array}$ & $\begin{array}{l}\text { Geometric } \\
\text { Mean }(\mathrm{Bq})\end{array}$ & $\begin{array}{l}\text { Geometric } \\
\text { Standard } \\
\text { Deviation }\end{array}$ \\
\hline $\mathrm{H}-3$ & $3.1 \mathrm{E}+12$ & 1.74 & Negligible & -- & $1.6 \mathrm{E}+09$ & 2.10 \\
\hline $\mathrm{C}-14$ & $1.3 \mathrm{E}+09$ & 2.12 & Negligible & -- & Negligible & -- \\
\hline Al-26 & $1.3 \mathrm{E}+03$ & 2.20 & Negligible & -- & Negligible & -- \\
\hline Ar-42 & $1.2 \mathrm{E}+07$ & 2.21 & Negligible & -- & Negligible & -- \\
\hline $\mathrm{K}-40$ & $4.0 \mathrm{E}+08$ & 2.22 & Negligible & -- & $4.2 E+03$ & 2.29 \\
\hline Ti-44 & $3.8 \mathrm{E}+08$ & 2.16 & Negligible & -- & Negligible & -- \\
\hline Co-60 & $2.0 \mathrm{E}+10$ & 2.02 & Negligible & -- & $6.7 \mathrm{E}+06$ & 2.23 \\
\hline $\mathrm{Ni}-63$ & $5.2 \mathrm{E}+10$ & 1.99 & Negligible & - & $4.9 E+07$ & 2.18 \\
\hline $\mathrm{Kr}-85$ & $2.2 \mathrm{E}+07$ & 1.94 & Negligible & -- & Negligible & -- \\
\hline Sr-90 & $5.3 E+10$ & 1.87 & $1.8 \mathrm{E}+07$ & 2.73 & $6.6 \mathrm{E}+09$ & 2.26 \\
\hline $\mathrm{Nb}-94$ & $8.7 E+03$ & 2.17 & Negligible & -- & Negligible & -- \\
\hline Tc-99 & $4.6 \mathrm{E}+12$ & 2.03 & $9.3 \mathrm{E}+08$ & 2.70 & $1.1 \mathrm{E}+11$ & 1.75 \\
\hline Sn-126 & Negligible & -- & Negligible & -- & $1.4 \mathrm{E}+07$ & 2.24 \\
\hline $\mathrm{I}-129$ & $4.8 \mathrm{E}-02$ & 2.13 & Negligible & -- & $1.2 \mathrm{E}+07$ & 2.67 \\
\hline Cs-137 & $5.3 \mathrm{E}+10$ & 1.84 & Negligible & -- & $8.4 \mathrm{E}+09$ & 2.07 \\
\hline Ba-133 & $5.1 E+04$ & 2.07 & Negligible & -- & Negligible & -- \\
\hline Sm-151 & $2.3 \mathrm{E}+06$ & 2.20 & Negligible & -- & Negligible & -- \\
\hline Eu-150 & 9.3E-01 & 2.13 & Negligible & -- & Negligible & -- \\
\hline Eu-152 & $2.3 \mathrm{E}+06$ & 1.73 & Negligible & -- & $1.2 \mathrm{E}+07$ & 2.22 \\
\hline Eu-154 & $3.4 \mathrm{E}+07$ & 2.00 & Negligible & -- & $1.5 \mathrm{E}+07$ & 2.25 \\
\hline Gd-152 & 1.4E-07 & 1.72 & Negligible & -- & 9.5E-07 & 2.21 \\
\hline $\mathrm{Pb}-210$ & $1.2 \mathrm{E}+09$ & 1.79 & $6.7 \mathrm{E}+09$ & 1.64 & $7.0 \mathrm{E}+10$ & 1.50 \\
\hline Ra-226 & $8.4 \mathrm{E}+08$ & 1.86 & $1.9 \mathrm{E}+10$ & 1.65 & $1.4 \mathrm{E}+11$ & 1.50 \\
\hline $\mathrm{Ra}-228$ & $4.9 \mathrm{E}+09$ & 1.82 & $5.9 E+12$ & 1.63 & $5.5 \mathrm{E}+12$ & 1.04 \\
\hline Ac-227 & $5.8 \mathrm{E}+07$ & 1.92 & $2.4 \mathrm{E}+06$ & 1.92 & $8.9 E+05$ & 1.71 \\
\hline Th-228 & $4.7 \mathrm{E}+09$ & 1.81 & $5.8 \mathrm{E}+12$ & 1.62 & $5.4 \mathrm{E}+12$ & 1.04 \\
\hline Th-229 & $2.6 \mathrm{E}+06$ & 1.87 & $5.0 E+09$ & 2.23 & $2.3 E+02$ & 1.98 \\
\hline Th-230 & $2.7 E+09$ & 1.53 & $1.5 \mathrm{E}+12$ & 1.67 & $2.0 \mathrm{E}+12$ & 2.08 \\
\hline Th-232 & $5.4 \mathrm{E}+09$ & 1.83 & $6.0 \mathrm{E}+12$ & 1.63 & $5.9 \mathrm{E}+12$ & 1.04 \\
\hline $\mathrm{Pa}-231$ & $1.6 \mathrm{E}+08$ & 1.84 & $6.5 \mathrm{E}+06$ & 1.92 & $3.4 \mathrm{E}+06$ & 1.72 \\
\hline $\mathrm{U}-232$ & $3.6 \mathrm{E}+07$ & 1.99 & Negligible & -- & $1.9 E+08$ & 2.20 \\
\hline U-233 & $3.1 \mathrm{E}+08$ & 1.99 & $1.9 \mathrm{E}+12$ & 2.22 & $2.1 E+05$ & 1.95 \\
\hline U-234 & $3.6 \mathrm{E}+12$ & 1.92 & $1.7 \mathrm{E}+11$ & 1.84 & $1.2 \mathrm{E}+11$ & 1.84 \\
\hline U-235 & $1.0 \mathrm{E}+11$ & 1.99 & $9.7 \mathrm{E}+09$ & 1.92 & $7.9 \mathrm{E}+09$ & 1.73 \\
\hline U-236 & $2.8 E+11$ & 1.98 & $1.7 \mathrm{E}+08$ & 2.25 & $1.4 \mathrm{E}+10$ & 1.94 \\
\hline
\end{tabular}




\begin{tabular}{|c|c|c|c|c|c|c|}
\hline \multirow{2}{*}{ Nuclide } & \multicolumn{2}{|c|}{ Pit 6 (Upper Cell) } & \multicolumn{2}{c|}{ Pit 6 (Lower Cell) RaDU } & \multicolumn{2}{c|}{ Pit 13 RaDU } \\
\cline { 2 - 7 } & $\begin{array}{c}\text { Geometric } \\
\text { Mean (Bq) }\end{array}$ & $\begin{array}{c}\text { Geometric } \\
\text { Standard } \\
\text { Deviation }\end{array}$ & $\begin{array}{c}\text { Geometric } \\
\text { Mean (Bq) }\end{array}$ & $\begin{array}{c}\text { Geometric } \\
\text { Standard } \\
\text { Deviation }\end{array}$ & $\begin{array}{c}\text { Geometric } \\
\text { Mean (Bq) }\end{array}$ & $\begin{array}{c}\text { Geometric } \\
\text { Standard } \\
\text { Deviation }\end{array}$ \\
\hline U-238 & $1.8 \mathrm{E}+13$ & 2.12 & $2.3 \mathrm{E}+11$ & 1.90 & $4.9 \mathrm{E}+11$ & 1.81 \\
\hline Np-237 & $2.4 \mathrm{E}+09$ & 1.92 & $7.6 \mathrm{E}+05$ & 2.68 & $2.1 \mathrm{E}+09$ & 1.93 \\
\hline $\mathrm{Pu}-238$ & $1.3 \mathrm{E}+10$ & 1.95 & $1.3 \mathrm{E}+10$ & 1.98 & $4.1 \mathrm{E}+08$ & 2.12 \\
\hline $\mathrm{Pu}-239$ & $1.4 \mathrm{E}+11$ & 1.64 & $3.3 \mathrm{E}+06$ & 2.07 & $9.0 \mathrm{E}+09$ & 1.98 \\
\hline $\mathrm{Pu}-240$ & $2.6 \mathrm{E}+10$ & 1.59 & Negligible & -- & $4.7 \mathrm{E}+07$ & 2.19 \\
\hline $\mathrm{Pu}-241$ & $8.1 \mathrm{E}+10$ & 1.73 & $1.2 \mathrm{E}+10$ & 2.23 & $6.4 \mathrm{E}+09$ & 2.14 \\
\hline $\mathrm{Pu}-242$ & $6.3 \mathrm{E}+06$ & 1.69 & Negligible & -- & $2.6 \mathrm{E}+03$ & 2.69 \\
\hline $\mathrm{Pu}-244$ & $4.3 \mathrm{E}+01$ & 1.95 & Negligible & -- & Negligible & -- \\
\hline $\mathrm{Am}-241$ & $2.7 \mathrm{E}+10$ & 1.50 & $1.1 \mathrm{E}+09$ & 2.24 & $1.5 \mathrm{E}+09$ & 1.78 \\
\hline Am-242m & $2.6 \mathrm{E}+05$ & 2.04 & Negligible & -- & Negligible & -- \\
\hline Am-243 & $4.8 \mathrm{E}+07$ & 1.94 & Negligible & -- & Negligible & -- \\
\hline $\mathrm{Cm}-243$ & $8.6 \mathrm{E}+07$ & 2.21 & Negligible & -- & Negligible & -- \\
\hline $\mathrm{Cm}-244$ & $2.8 \mathrm{E}+08$ & 1.96 & Negligible & -- & Negligible & -- \\
\hline $\mathrm{Cm}-245$ & $7.6 \mathrm{E}+05$ & 2.25 & Negligible & -- & Negligible & -- \\
\hline $\mathrm{Cm}-246$ & $3.3 \mathrm{E}+00$ & 2.10 & Negligible & -- & Negligible & -- \\
\hline $\mathrm{Cm}-247$ & $1.1 \mathrm{E}+06$ & 2.15 & Negligible & -- & Negligible & -- \\
\hline $\mathrm{Cm}-248$ & $7.1 \mathrm{E}+05$ & 2.14 & Negligible & -- & Negligible & -- \\
\hline $\mathrm{Cf}-249$ & $6.2 \mathrm{E}+04$ & 2.02 & Negligible & -- & Negligible & -- \\
\hline Total & $3.0 \mathrm{E}+13$ & & $\mathbf{2 . 2 E + 1 3}$ & & $\mathbf{2 . 0 E + 1 3}$ & \\
\hline
\end{tabular}

Negligible - Inventory less than $37 \mathrm{~Bq}$

\section{GCD Inventories}

The GCD boreholes have received high specific activity waste, including TRU waste regulated under 40 CFR 191. The GCD boreholes were active from FY 1984 through FY 1990. The PA divides the GCD inventory into pre- and post-1988 portions. The majority of the waste on an activity and volume basis was disposed in the pre-1988 period. The current GCD inventory estimates are summarized Table 11. The GCD inventories are not significantly different from previous estimates.

Table 11. FY 2012 Estimate of the Area 5 RWMS GCD Borehole Inventory (Estimates are calculated from 500 LHS realizations and decayed to October 1, 2028)

\begin{tabular}{|c|c|c|c|c|}
\hline \multirow{2}{*}{ Nuclide } & \multicolumn{2}{|c|}{ Pre-1988 GCD } & \multicolumn{2}{c|}{ Post-1988 GCD } \\
\cline { 2 - 5 } & $\begin{array}{c}\text { Geometric Mean } \\
(\mathbf{B q})\end{array}$ & $\begin{array}{c}\text { Geometric } \\
\text { Standard Deviation }\end{array}$ & $\begin{array}{c}\text { Geometric Mean } \\
\mathbf{( B q )}\end{array}$ & $\begin{array}{c}\text { Geometric } \\
\text { Standard Deviation }\end{array}$ \\
\hline $\mathrm{H}-3$ & $2.1 \mathrm{E}+16$ & 2.24 & $1.8 \mathrm{E}+14$ & 2.18 \\
\hline $\mathrm{C}-14$ & $7.2 \mathrm{E}+04$ & 2.65 & Negligible & -- \\
\hline $\mathrm{Al}-26$ & $2.7 \mathrm{E}+00$ & 2.67 & Negligible & -- \\
\hline $\mathrm{Cl}-36$ & $1.6 \mathrm{E}+04$ & 2.63 & Negligible & -- \\
\hline
\end{tabular}




\begin{tabular}{|c|c|c|c|c|}
\hline \multirow[b]{2}{*}{ Nuclide } & \multicolumn{2}{|c|}{$\begin{array}{l}\text { Pre-1988 GCD } \\
\end{array}$} & \multicolumn{2}{|c|}{ Post-1988 GCD } \\
\hline & $\begin{array}{c}\text { Geometric Mean } \\
(\mathrm{Bq})\end{array}$ & $\begin{array}{c}\text { Geometric } \\
\text { Standard Deviation }\end{array}$ & $\begin{array}{c}\text { Geometric Mean } \\
\text { (Bq) }\end{array}$ & $\begin{array}{c}\text { Geometric } \\
\text { Standard Deviation }\end{array}$ \\
\hline Ar-39 & $7.4 \mathrm{E}+04$ & 2.67 & Negligible & -- \\
\hline $\mathrm{K}-40$ & $4.2 E+03$ & 2.57 & Negligible & -- \\
\hline Ca-41 & $1.1 \mathrm{E}+05$ & 2.71 & Negligible & -- \\
\hline Co-60 & $9.8 \mathrm{E}+11$ & 2.49 & Negligible & -- \\
\hline $\mathrm{Ni}-59$ & $2.9 \mathrm{E}+03$ & 2.66 & Negligible & -- \\
\hline $\mathrm{Ni}-63$ & $2.5 \mathrm{E}+05$ & 2.70 & Negligible & -- \\
\hline Kr-85 & $6.5 E+04$ & 2.60 & Negligible & -- \\
\hline Sr-90 & $5.2 E+15$ & 3.68 & $1.3 E+08$ & 3.99 \\
\hline Zr-93 & $3.9 \mathrm{E}+02$ & 2.57 & Negligible & -- \\
\hline $\mathrm{Nb}-93 \mathrm{~m}$ & $6.7 \mathrm{E}+04$ & 2.82 & Negligible & -- \\
\hline $\mathrm{Nb}-94$ & $9.5 \mathrm{E}+04$ & 2.63 & Negligible & -- \\
\hline Tc-99 & $7.7 \mathrm{E}+09$ & 3.22 & $7.3 \mathrm{E}+09$ & 3.69 \\
\hline $\mathrm{Pd}-107$ & $1.8 \mathrm{E}+01$ & 2.59 & Negligible & -- \\
\hline Cd-113m & $6.2 \mathrm{E}+04$ & 2.74 & Negligible & -- \\
\hline Sn-121m & $1.0 \mathrm{E}+06$ & 2.71 & Negligible & -- \\
\hline Sn-126 & $1.7 \mathrm{E}+02$ & 2.57 & Negligible & -- \\
\hline $\mathrm{I}-129$ & $9.0 \mathrm{E}+00$ & 2.57 & Negligible & -- \\
\hline Cs-135 & $3.1 \mathrm{E}+02$ & 2.59 & Negligible & -- \\
\hline Cs-137 & $2.8 \mathrm{E}+14$ & 3.75 & Negligible & -- \\
\hline Sm-151 & $4.0 \mathrm{E}+05$ & 2.58 & Negligible & -- \\
\hline Eu-150 & $1.5 \mathrm{E}+05$ & 2.82 & Negligible & -- \\
\hline Eu-152 & 4.7E+05 & 2.67 & Negligible & -- \\
\hline Eu-154 & $9.9 \mathrm{E}+04$ & 2.75 & Negligible & -- \\
\hline Gd-152 & 1.1E-07 & 2.66 & Negligible & -- \\
\hline Ho-166m & $3.7 \mathrm{E}+03$ & 2.66 & Negligible & -- \\
\hline $\mathrm{Pb}-210$ & $2.7 \mathrm{E}+12$ & 4.11 & $4.2 \mathrm{E}+04$ & 2.24 \\
\hline Ra-226 & $3.6 \mathrm{E}+12$ & 4.11 & $1.3 \mathrm{E}+05$ & 2.24 \\
\hline $\mathrm{Ra}-228$ & $1.1 \mathrm{E}+09$ & 3.07 & 3.1E-08 & 3.84 \\
\hline Ac-227 & $7.2 \mathrm{E}+10$ & 4.28 & $5.9 \mathrm{E}+05$ & 2.31 \\
\hline Th-228 & $1.0 \mathrm{E}+09$ & 3.07 & 2.7E-08 & 3.84 \\
\hline Th-229 & $8.7 \mathrm{E}+01$ & 1.90 & $5.1 \mathrm{E}+01$ & 2.20 \\
\hline Th-230 & $5.7 \mathrm{E}+07$ & 2.89 & $1.6 \mathrm{E}+07$ & 2.24 \\
\hline Th-232 & $1.1 \mathrm{E}+09$ & 3.07 & 4.7E-08 & 3.84 \\
\hline $\mathrm{Pa}-231$ & $4.8 \mathrm{E}+06$ & 2.86 & $1.4 \mathrm{E}+06$ & 2.31 \\
\hline $\mathrm{U}-232$ & $4.4 \mathrm{E}+03$ & 2.67 & Negligible & -- \\
\hline U-233 & $4.2 \mathrm{E}+04$ & 1.92 & $2.7 \mathrm{E}+04$ & 2.20 \\
\hline U-234 & $1.4 \mathrm{E}+11$ & 2.87 & $4.3 \mathrm{E}+10$ & 2.24 \\
\hline U-235 & $5.2 \mathrm{E}+09$ & 2.85 & $1.6 \mathrm{E}+09$ & 2.31 \\
\hline
\end{tabular}




\begin{tabular}{|c|c|c|c|c|}
\hline \multirow{2}{*}{ Nuclide } & \multicolumn{2}{|c|}{ Pre-1988 GCD } & \multicolumn{2}{c|}{ Post-1988 GCD } \\
\cline { 2 - 5 } & $\begin{array}{c}\text { Geometric Mean } \\
\mathbf{( B q})\end{array}$ & $\begin{array}{c}\text { Geometric } \\
\text { Standard Deviation }\end{array}$ & $\begin{array}{c}\text { Geometric Mean } \\
\mathbf{( B q})\end{array}$ & $\begin{array}{c}\text { Geometric } \\
\text { Standard Deviation }\end{array}$ \\
\hline $\mathrm{U}-236$ & $3.6 \mathrm{E}+08$ & 3.63 & $4.8 \mathrm{E}+01$ & 3.84 \\
\hline $\mathrm{U}-238$ & $4.0 \mathrm{E}+10$ & 2.54 & $7.8 \mathrm{E}+10$ & 2.20 \\
\hline Np-237 & $2.6 \mathrm{E}+08$ & 1.99 & $1.6 \mathrm{E}+08$ & 2.20 \\
\hline Pu-238 & $2.9 \mathrm{E}+11$ & 3.21 & $3.4 \mathrm{E}+06$ & 3.79 \\
\hline Pu-239 & $1.8 \mathrm{E}+13$ & 3.08 & $1.9 \mathrm{E}+08$ & 3.80 \\
\hline Pu-240 & $3.6 \mathrm{E}+12$ & 3.24 & $4.1 \mathrm{E}+07$ & 3.84 \\
\hline Pu-241 & $4.1 \mathrm{E}+12$ & 3.48 & $5.9 \mathrm{E}+07$ & 4.01 \\
\hline Pu-242 & $3.5 \mathrm{E}+08$ & 3.20 & Negligible & -- \\
\hline Am-241 & $5.8 \mathrm{E}+12$ & 2.59 & $3.6 \mathrm{E}+07$ & 3.80 \\
\hline Am-243 & $3.6 \mathrm{E}+01$ & 2.60 & Negligible & -- \\
\hline Cm-244 & $8.0 \mathrm{E}+03$ & 2.58 & Negligible & -- \\
\hline Total & $\mathbf{2 . 7 E + 1 6}$ & & $1.9 \mathrm{E}+14$ & \\
\hline
\end{tabular}

Negligible - Inventory less than $37 \mathrm{~Bq}$

\subsubsection{Waste Acceptance Criteria}

WAC for the Area 3 and Area 5 RWMSs are described in Nevada National Security Site Waste Acceptance Criteria (NNSA/NSO 2012a). Revision 9 of the NNSS WAC was released in FY 2012. The only substantive change is the acceptance of non-radioactive classified waste and non-radioactive hazardous classified waste. Non-radioactive waste must be classified and meet all applicable NNSS WAC requirements. Non-radioactive waste volume in FY 2012 was an insignificant fraction of the total waste volume disposed. WAC action levels are PA derived waste concentrations used to screen waste streams for their potential to impact PA results. WAC action levels are unchanged and continue to be based on PA results.

Compliance with the NNSS WAC is ensured by the RWAP, an NNSA/NSO program, which reviews and approves all new or revised waste streams and generator waste certification programs (NNSA/NSO 2006). No changes occurred to the RWAP in FY 2012.

\subsection{FACILITY DESIGN}

Key facility design features are specifications impacting PA conceptual models, assumptions, or input parameters. Key facility design features include the following:

- Disposal unit volume, area, and depth below grade

- Disposal unit engineered barrier design and condition

- Controls that impact and compensate for subsidence

\subsubsection{Disposal Unit Design}

The Area 3 RWMS uses nuclear subsidence craters as waste disposal units. The Area 3 RWMS was placed in inactive status in July 2006, with the last waste disposed in April 2006. The two 
post-1988 disposal units, U-3ah/at and U-3bh, are currently operationally closed. No wastes were disposed at the Area 3 RWMS and no new disposal units were opened in FY 2012. Area 3 RWMS disposal unit design continues to be consistent with the PA model.

Radioactive waste is currently disposed at the Area 5 RWMS in shallow unlined pits and trenches. RCRA-compliant mixed waste is disposed in a double lined cell with a leachate collection system. In the past, ${ }^{222} \mathrm{Rn}$-generating waste was disposed in deeper disposal units with thicker covers known as RaDUs, and high specific activity waste was disposed in intermediate depth GCD boreholes.

A small slit trench, Trench 13, was opened between Pit 13 and Pit 17 for the disposal of high exposure rate waste in FY 2012. The depth and cover thickness of Trench 13 are consistent with other SLB disposal units. No other disposal units were added in FY 2012. Area 5 RWMS disposal unit design continues to be consistent with the PA model.

\subsubsection{Engineered Barriers}

Engineered barriers at the Area 3 and Area 5 RWMSs include flood control berms, the closure cover, and the liner and leachate collection system for the Pit 18 mixed waste disposal unit at the Area 5 RWMS. The Area 3 and Area 5 RWMS flood control berms and closure covers are described in the PAs and closure plans. The Area 3 and Area 5 RWMS closure cover designs were unchanged in FY 2012. The Area 5 RWMS Pit 18 liner and leachate collection system was described in the FY 2010 Annual Summary Report (NSTec 2011). The Pit 18 liner and leachate collection system design is unchanged.

\subsubsection{Environmental Monitoring}

Monitoring activities at the Area 3 and 5 RWMSs and at the NNSS provide the data necessary to support PA and CA maintenance. The Nevada Test Site Routine Radiological Environmental Monitoring Plan (BN 2003) is the basis for all NNSS-wide environmental surveillance, site-specific effluent monitoring, and operational monitoring conducted by various missions, programs, and projects. The monitoring plan is in final form. Closure plans for the Area 3 RWMS and Area 5 RWMS (NSTec 2007b, 2008a) describe the specific monitoring programs for the waste disposal facilities. No significant changes occurred in the environmental monitoring plan in FY 2012.

Current monitoring activities at the Area 3 and Area 5 RWMS are summarized in Table 12. Three minor operational changes occurred in FY 2012. The number of air sampling stations at the Area 3 RWMS was reduced from four to three, and the sampling locations were moved. Monitoring of ${ }^{3} \mathrm{H}$ in soil pore gas was discontinued at the Area 5 RWMS GCD boreholes with final closure of the 37-hectare (92-acre [ac]) Low-Level Waste Management Unit (LLWMU). The elevation of the 92-ac LLWMU final closure cover is now surveyed annually to monitor subsidence. Automated vadose zone water content monitoring of Area 5 RWMS operational covers and pit floors was temporarily suspended during calendar year (CY) 2011 due to final closure cover construction operations at the 92-ac LLWMU. 
Table 12. Summary of Area 3 and Area 5 RWMS Monitoring Programs

\begin{tabular}{|c|c|c|}
\hline Monitoring Element & Area 3 RWMS & Area 5 RWMS \\
\hline Vadose Zone Monitoring & $\begin{array}{l}\text { Measurements of soil water } \\
\text { content in waste disposal unit } \\
\text { cover } \\
\text { - Eight drainage lysimeters for } \\
\text { water balance since } 2001\end{array}$ & $\begin{array}{l}\text { - Measurements of soil water } \\
\text { content and water potential in } \\
\text { waste disposal unit covers } \\
\text { (Temporarily suspended) } \\
\text { - Measurements of soil water } \\
\text { content in waste disposal unit } \\
\text { floor (Temporarily suspended) } \\
\text { - Two weighing lysimeters } \\
\text { (vegetated and bare) for water } \\
\text { balance in operation since } 1994\end{array}$ \\
\hline Groundwater Monitoring & - None & $\begin{array}{l}\text { - RCRA detection monitoring at } \\
\text { three wells }\end{array}$ \\
\hline Radon Monitoring & $\begin{array}{l}\text { Radon flux measurements from } \\
\text { waste covers (various locations) }\end{array}$ & 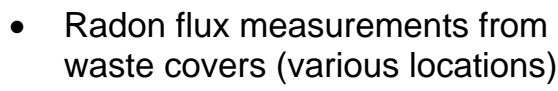 \\
\hline Meteorology Monitoring & $\begin{array}{l}\text { - } \text { Air temperature at } 3 \text { and } 10 \mathrm{~m} \\
\text { - }(10 \text { and } 33 \mathrm{ft} \text { ) } \\
\text { - } \text { Relative humidity at two heights } \\
\text { - } \text { Wind direction at two heights } \\
\text { - } \text { Barometric pressure } \\
\text { - } \quad \text { Solar radiation } \\
\text { - } \text { Precipitation }\end{array}$ & $\begin{array}{l}\text { - } \quad \text { Air temperature at } 3 \text { and } 10 \mathrm{~m} \\
\text { - } 10 \text { and } 33 \mathrm{ft}) \\
\text { - } \text { Relative humidity at two heights } \\
\text { - } \quad \text { Wind speed at two heights } \\
\text { - } \quad \text { Wind direction at two heights } \\
\text { - } \quad \text { Barometric pressure } \\
\text { - } \quad \text { Solar radiation } \\
\text { - } \text { Precipitation }\end{array}$ \\
\hline Direct Radiation Monitoring & $\begin{array}{l}\text { - Nine thermoluminescent } \\
\text { dosimeters (TLDs) }\end{array}$ & - Ten TLDs \\
\hline Biota Monitoring & $\begin{array}{l}\text { Sampling vegetation, small } \\
\text { mammals, and animal burrow } \\
\text { spoils for tritium, gamma- } \\
\text { emitting radionuclides, }{ }^{90} \mathrm{Sr} \text {, } \\
\text { americium-241 }\left({ }^{241} \mathrm{Am}\right) \text {, and } \\
\text { plutonium }\end{array}$ & $\begin{array}{l}\text { - Sampling vegetation, small } \\
\text { mammals, and animal burrow } \\
\text { spoils for tritium, gamma- } \\
\text { emitting radionuclides, }{ }^{90} \mathrm{Sr} \text {, } \\
{ }^{241} \mathrm{Am} \text {, and plutonium }\end{array}$ \\
\hline Subsidence Monitoring & $\begin{array}{l}\text { - Routine inspection of } \\
\text { operational covers }\end{array}$ & $\begin{array}{l}\text { - } \begin{array}{l}\text { Routine inspection of } \\
\text { operational covers }\end{array} \\
\text { - } \\
\text { 92-ac LLWMU closure cover } \\
\text { surveyed annually }\end{array}$ \\
\hline Air Monitoring & $\begin{array}{l}\text { Atmospheric moisture sampling } \\
\text { for tritium and air particulates } \\
\text { sampled at three locations }\end{array}$ & $\begin{array}{l}\text { - Air particulates sampled at two } \\
\text { locations; atmospheric moisture } \\
\text { sampling for tritium at two } \\
\text { locations }\end{array}$ \\
\hline $\begin{array}{l}\text { Soil Temperature Monitoring } \\
\text { around RTGs }\end{array}$ & - None & $\begin{array}{l}\text { - Vertical and horizontal sensor } \\
\text { arrays around four RTGs in } \\
\text { Pit } 5\end{array}$ \\
\hline
\end{tabular}


Environmental monitoring data are reported on a CY basis. The following four reports, published annually, contain details regarding the monitoring program and results for CY 2011:

- Nevada National Security Site Environmental Report (NSTec 2012a)

- National Emission Standards for Hazardous Air Pollutants Report (NSTec 2012b)

- Waste Management Monitoring Report (NSTec 2012c)

- Area 5 Groundwater Monitoring Report (NSTec 2013)

Results of the environmental monitoring programs were consistent with PA input parameters and model results (NSTec 2012c). CY 2011 monitoring results are consistent with trends observed in previous years.

\subsubsection{Stability Control}

Subsidence is minimized and controlled by WAC and site operations. The NNSS WAC requires that waste packages be loaded to ensure that the interior space is loaded as compactly and as efficiently as practicable. Site operations minimize subsidence by carefully planning waste placement and by monitoring and repairing subsidence detected on closed disposal units. Cover elevation is surveyed annually on units that have undergone final closure. No changes to these procedures occurred in FY 2012.

\subsection{CLOSURE DESIGN}

\subsubsection{Closure Plan}

The approved Area 3 RWMS PA/CA assumes that the site will be closed with a vegetated monolithic evapotranspirative (ET) cover of native alluvium. The cover is assumed to be $3 \mathrm{~m}$ (10 ft) thick after subsidence. The Closure Plan for the Area 3 Radioactive Waste Management Site at the Nevada Test Site is in final form (NSTec 2007b). The cover design is for a $3 \mathrm{~m}$ (10 ft) monolithic ET cover (NSTec 2007b), consistent with the Area 3 RWMS PA/CA. The Area 3 RWMS PA and CA assumptions continue to be consistent with completed closures and closure plans.

Closure plans for the Area 5 RWMS have evolved over time based on the documented results of PA modeling. The most recently approved PA version, the 2006 Area 5 RWMS PA update (BN

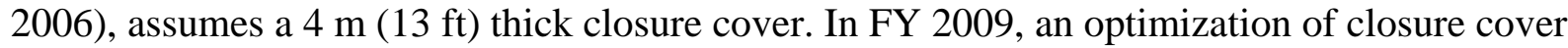
thickness was performed for the 92-ac LLWMU, the northern expansion area, and the entire Area 5 RWMS (Shott and Yucel 2009). The optimization used cost-benefit analysis to select the optimum cover thickness, ranging from 2.5 to $4.5 \mathrm{~m}$ (8.2 to $15 \mathrm{ft}$ ). Each cover option was constrained to meet all performance objectives and CA requirements in DOE M 435.1-1 (DOE 1999b). The cost of collective dose averted was found to be small relative to cover construction costs. The optimum cover that meets all PA and CA requirements was found to be the $2.5 \mathrm{~m}$ (8.2 ft) cover. The current Area 5 RWMS v4.114 GoldSim model assumes a $2.5 \mathrm{~m}(8.2 \mathrm{ft})$ cover.

The Closure Plan for the Area 5 Radioactive Waste Management Site at the Nevada Test Site is in final form (NSTec 2008a). Closure of the Area 5 RWMS is planned in two phases. The first 
phase is closure of the 92-ac LLWMU under the Federal Facility Agreement and Consent Order (FFACO) closure process. A Corrective Action Decision Document/Corrective Action Plan (CADD/CAP) for the 92-ac LLWMU (Corrective Action Unit [CAU] 111) was approved by the Nevada Division of Environmental Protection (NDEP) in FY 2009 (NNSA/NSO 2009a). The preferred Corrective Action Alternative, a $2.5 \mathrm{~m}$ (8.2 ft) thick engineered monolithic ET cover, was constructed in FY 2011. The Closure Report for the 92-ac LLWMU was issued in FY 2012 (NNSA/NSO 2012b). Re-vegetation of the closure cover was initiated FY 2012.

The second phase, closure of the northern expansion area, is scheduled for FY 2028. The current Area 5 RWMS closure plan is to close the northern expansion area with a monolithic ET cover. The final cover thickness will be determined by future PA modeling when the final closure inventory is known. Area 5 RWMS closure plans continue to be consistent with PA modeling results.

\subsubsection{Institutional Control Policy}

The NNSA/NSO institutional control policy states that institutional controls will be implemented to maintain and enforce restricted access to, and use of, the NNSS and ensure the continuity of appropriate institutional controls in the future (NNSA/NSO 2008). Based on the institutional control policy, PA/CA analyses assume implementation of land-use restrictions consistent with the UGTA FFACO closure strategies for the NNSS (NNSA/NSO 2007). The planned land-use restrictions will prohibit public access to groundwater for 1,000 years within the use restriction boundaries negotiated with the State of Nevada.

Although the final regulatory boundaries have not been negotiated, the Area 3 RWMS and Area 5 RWMS are expected to be within the boundaries of the Yucca Flat CAU 97 and the Frenchman Flat CAU 98, respectively. The NNSA/NSO Assistant Manager of Environmental Management has administratively agreed to include the Area 5 RWMS within the CAU 98 use restriction boundaries (NNSA/NSO 2008). The Area 5 RWMS is currently within the initial Frenchman Flat CAU 98 use restriction boundaries.

The institutional control policy has changed PA analyses in the following areas:

1) Long-term (i.e., chronic) exposure of intruders is assumed to be impossible based on NNSS land-use restrictions and planned UGTA groundwater-use restrictions.

2) Short-term or acute intruder exposure may occur.

3) Exposure of the member of public and short-term exposure of intruders is assumed possible after institutional controls end. The period of institutional control will be randomly sampled from a probability density function. The member of public will be located at the use restriction boundary.

4) The institutional control policy and the probabilistic period of institutional controls are not applied to the 40 CFR 191.13 containment requirements, which do not allow PAs to assume institutional control is effective beyond 100 years. 
These changes are implemented in the current Area 3 RWMS PA and Area 5 RWMS PA GoldSim models except for changing the point of compliance to the use restriction boundaries.

\subsection{RESEARCH AND DEVELOPMENT}

The PA/CA Maintenance Plan calls for annual reviews of R\&D activities relevant to the PA. Onsite and offsite R\&D activities (e.g., those performed at other DOE sites, the national laboratories, the Desert Research Institute, and academic institutions) provide the data used to evaluate uncertainty in conceptual models, mathematical models, and model parameters and to ensure continuing adequacy of the PA.

The DASs require NNSA/NSO to address all secondary issues (e.g., consistency of models and parameters between the Area 3 and Area 5 RWMSs) noted during the PA/CA reviews as part of the maintenance program. R\&D is the mechanism for NNSA/NSO to address these issues and manage uncertainty.

No confirmatory testing is conducted under the R\&D program. The environmental monitoring program includes measurement and monitoring of numerous parameters (e.g., vadose zone moisture contents, radionuclide concentrations in air and groundwater) that confirm the performance of the RWMSs and continuing adequacy of the PA.

\subsubsection{FY 2012 R\&D Activities}

The major R\&D efforts undertaken in FY 2012 were the further development of the GoldSim models supporting the Area 3 RWMS and Area 5 RWMS PAs and CAs. Model development activities are performed to maintain consistency with known site conditions (e.g., site inventory, monitoring results), improve consistency between the Area 3 and Area 5 RWMS PA/CA models, and reduce model uncertainty.

\section{Area 5 RWMS PA GoldSim Model Development}

A new FY 2012 baseline version of the Area 5 RWMS model, 4.114, has been approved for all model applications, including waste stream evaluations and compliance determinations (NNSA/NSO 2013). The FY 2012 PA update was performed with the Area 5 RWMS v4.114 PA model. Major developments since version 4.113 of the model include the following:

- All inventories and disposal unit dimensions are updated to FY 2012 estimates.

\section{Area 3 RWMS GoldSim Model Development}

The current baseline version of the Area 3 RWMS model, 2.102, was approved for all model applications in FY 2011 (NNSA/NSO 2011a). Major developments since version 2.000 of the model include:

- All inventories are updated through July 2006 when the site was placed in standby.

- A resident exposure scenario was added to the model. The resident scenario is equivalent to the community without agriculture scenario in the approved PA. 
- Acute intruder scenarios are added to the model.

- A revised subsidence model is included for the U-3ah/at and U-3bh disposal units. The new model, based on additional review of waste form data, is probabilistic and dynamic.

- Internal and external radiological dose conversion factors were updated with adult dose conversion factors from the Federal Guidance Report 13 Supplemental CD

(U.S. Environmental Protection Agency [EPA] 1999).

- Revised estimates of disposal unit volume, area, and depth are used.

The special analysis for the Area 3 RWMS was prepared using the Area 3 RWMS v2.102 model (NSTec 2012d). A new baseline model was not developed in FY 2012.

Area 5 RWMS Inventory GoldSim Model Development

The Area 5 RWMS FY 2012 inventory estimate was prepared with the Area 5 Inventory v2.110 model. The only major change from the previous version is the addition of FY 2012 disposal data.

\section{Area 3 RWMS Inventory GoldSim Model Development}

The Area 3 RWMS FY 2012 inventory estimate was prepared with the Area 3 Inventory v2.016 model. No changes were made to the Area 3 inventory model in FY 2012.

\subsubsection{Future R\&D Activities}

The long-term goal of the maintenance program is to reduce uncertainty in exposure scenarios (member of public and inadvertent human intrusion), conceptual models, mathematical models, and model parameters. Reduction of uncertainty and associated improvement of the PA model will be accomplished through special studies. In addition, future R\&D activities include the development of new waste concentration limits, evaluation of waste forms and containers (both engineering and geochemical properties) for disposal, the refinement of closure cover designs, and evaluation of institutional control and land-use options for optimizing disposal operations.

In December 2005, the EPA adopted American Meteorological Society/EPA Regulatory Model (AERMOD) as the preferred regulatory compliance code to replace the Industrial Source Code 3 (ISC3) for near-field, less than 50 kilometers (31 miles), atmospheric dispersion modeling (EPA 2005). The ISC3 code was used for atmospheric dispersion modeling in the Area 3 RWMS and Area 5 RWMS CAs. In FY 2013, AERMOD will be investigated as a replacement for atmospheric dispersion modeling in the Area 3 RWMS and Area 5 RWMS PA/CA models.

\subsection{SUMMARY OF CHANGES}

Waste operations, facility design, monitoring results, and $R \& D$ results for the Area 3 and Area 5 RWMSs were reviewed to identify changes potentially impacting the PAs and the DASs. Discovered and proposed changes are summarized below. 


\subsubsection{Discovered Changes}

There are no discovered changes for the Area 3 RWMS and Area 5 RWMS PAs in FY 2012.

\subsubsection{Proposed Changes}

\subsubsection{Area 3 RWMS}

The Area 3 RWMS was inactive in FY 2012. No significant changes related to operations, facility design, or inventory occurred in FY 2012. A new baseline PA/CA model was released and used to perform a special analysis. Changes to the PA model are described in the special analysis and summarized below. Review of the maintenance plan, closure plan, and monitoring plan indicate that no changes or revisions are necessary.

\subsubsection{Area 5 RWMS}

Facility changes occurred at the Area 5 RWMS in FY 2012. A new SLB disposal unit began disposal operations. Additional inventory was disposed in FY 2012, including inventory from five new or revised waste streams that required a special analysis for acceptance. Minor operational changes occurred to the Area 5 RWMS monitoring program. A new baseline PA/CA model was released. Review of the maintenance plan, closure plan, and monitoring plan indicate that no changes or revisions are necessary.

\subsubsection{R\&D Changes}

\subsubsection{Area 3 RWMS}

In FY 2012, a special analysis was prepared for the Area 3 RWMS using a new baseline PA/CA model, version 2.102 (NSTec 2012d). Prior annual summary reports have documented multiple changes occurring since preparation of the PA and CA. Potentially important changes include the following:

- Development of a new and improved baseline PA and CA model implemented in the probabilistic GoldSim simulation platform

- A significant increase in the waste inventory disposed at the site

- Revision and updating of model parameters based on additional years of site monitoring data and new research and development results

Although changes have occurred, many important PA/CA issues remain unchanged, including the site conceptual model, important FEPs, and the points of compliance. The special analysis was performed to document the current status of the PA/CA model and to quantitatively assess the impact of cumulative changes on the PA and CA results. The results of the special analysis are used to assess the validity of the approved PA/CA and make a determination if revision of the PA or CA is necessary.

The Area 3 RWMS special analysis used the Area 3 RWMS v2.102 GoldSim model to assess the continuing validity of PA conclusions. The geometric mean inventory and standard deviation data listed in Tables 7 and 8 were entered into the inventory elements for U-3ax/bl, U-3ah/at, and 
U-3bh. The disposal unit area, disposal unit volume, and waste volumes were updated with current data. All disposal units were assumed to be closed with a $3 \mathrm{~m}(9.8 \mathrm{ft})$ thick cover. The model was run assuming a median period of active institutional control of 245 years, a 100-year period of passive institutional control, and a 1,000-year compliance period. The model was run in GoldSim version 10.5(SP2) with 5,000 LHS realizations.

Comparison of the maximum special analysis results with the PA performance objectives indicates that there continues to be a reasonable expectation of compliance with the performance objectives. The resident exposure scenario was evaluated for compliance with the air pathway performance objective (Table 13). The maximum mean air pathway annual total effective dose (TED) for a resident, 7E-6 millisievert (mSv) at 1,000 years, has decreased relative to the approved PA and is a small fraction of the $0.1 \mathrm{mSv}$ limit. The mean and $95^{\text {th }}$ percentile air pathway annual TED results are less than the performance objective for all scenarios. The air pathway annual TED is due predominantly to inhalation of lead-210 $\left({ }^{210} \mathrm{~Pb}\right)$ for all scenarios at 1,000 years. At closure, ${ }^{3} \mathrm{H}$ is the predominant source of air pathway TED.

Table 13. Area 3 RWMS v2.102 GoldSim Model Member of Public Total Annual TED through the Air Pathway

\begin{tabular}{|c|c|c|c|}
\hline Scenario & $\begin{array}{c}\text { Mean Annual TED } \\
(\mathbf{m S v})\end{array}$ & $\begin{array}{c}\mathbf{9 5}^{\text {th }} \text { Percentile } \\
(\mathbf{m S v})\end{array}$ & $\begin{array}{c}\text { Time of } \\
\text { Maximum }\end{array}$ \\
\hline Resident & $6.5 \mathrm{E}-6$ & $1.7 \mathrm{E}-5$ & 1,000 years \\
\hline Resident with Agriculture & $7.3 \mathrm{E}-6$ & $1.9 \mathrm{E}-5$ & 1,000 years \\
\hline Transient Occupant & $2.6 \mathrm{E}-6$ & $7.3 \mathrm{E}-6$ & 1,000 years \\
\hline
\end{tabular}

The maximum mean resident all-pathways annual TED, 7E-5 mSv at 1,000 years, has increased relative to the approved PA but remains a small fraction of the $0.25 \mathrm{mSv}$ limit (Table 14). The mean and $95^{\text {th }}$ percentile all-pathways annual TED results are a small fraction of the performance objective for all scenarios. The resident all-pathways annual TED is due predominantly to ${ }^{210} \mathrm{~Pb}$. The resident with agriculture all-pathways TED is due predominantly to ${ }^{210} \mathrm{~Pb}$ and ${ }^{99} \mathrm{Tc}$. The transient occupant all-pathways TED is due predominantly to external exposure from ${ }^{222} \mathrm{Rn}$ progeny in cover soil.

Table 14. Area 3 RWMS v2.102 GoldSim Model Member of Public Total Annual TED through All-Pathways

\begin{tabular}{|c|c|c|c|}
\hline Scenario & $\begin{array}{c}\text { Mean Annual TED } \\
(\mathbf{m S v})\end{array}$ & $\begin{array}{c}\mathbf{9 5}^{\text {th }} \text { Percentile } \\
(\mathbf{m S v})\end{array}$ & $\begin{array}{c}\text { Time of } \\
\text { Maximum }\end{array}$ \\
\hline Resident & $7.1 \mathrm{E}-5$ & $2.0 \mathrm{E}-4$ & 1,000 years \\
\hline Resident with Agriculture & $1.2 \mathrm{E}-3$ & $3.6 \mathrm{E}-3$ & 1,000 years \\
\hline Transient Occupant & $1.3 \mathrm{E}-3$ & $3.4 \mathrm{E}-3$ & 1,000 years \\
\hline
\end{tabular}

The maximum mean ${ }^{222} \mathrm{Rn}$ flux density, 0.03 becquerels per square meter per second $\left(\mathrm{Bq} \mathrm{m} \mathrm{m}^{-2} \mathrm{~s}^{-1}\right)$, has increased relative to the PA results but is significantly less than the $0.74 \mathrm{~Bq} \mathrm{~m}^{-2} \mathrm{~s}^{-1}$ limit. The mean and $95^{\text {th }}$ percentile ${ }^{222} \mathrm{Rn}$ flux density are less than the performance objective throughout the 1000-year compliance period (Table 15). 
Table 15. Area 3 RWMS v2.102 GoldSim Model ${ }^{222}$ Rn Flux Density

\begin{tabular}{|c|c|c|c|}
\hline Disposal Unit & $\begin{array}{c}\text { Mean }{ }^{222} \text { Rn Flux Density } \\
\left(\mathrm{Bq} \mathrm{m}^{-2} \mathbf{~ s}^{-1}\right)\end{array}$ & $\begin{array}{c}95^{\text {th }} \text { Percentile } \\
\left(\mathrm{Bq} \mathrm{m}^{-2} \mathbf{s}^{-1}\right)\end{array}$ & $\begin{array}{c}\text { Time of } \\
\text { Maximum }\end{array}$ \\
\hline U-3ah/at and U-3bh & 0.029 & 0.070 & 1,000 years \\
\hline
\end{tabular}

The special analysis estimates intruder doses for acute intruder scenarios only. The Area 3 RWMS is expected to be located within the Yucca Flat CAU 97 use restriction boundaries. Based on NNSA/NSO institutional control polices, chronic intrusion is assumed to be unlikely for 1,000 years. The mean and $95^{\text {th }}$ percentile acute drilling intruder TEDs are a small fraction of the $5 \mathrm{mSv}$ performance objective for both post-1988 disposal units (Table 16). Acute intruder doses were not estimated in the approved PA. The acute drilling TED is due predominantly to ${ }^{222} \mathrm{Rn}$, uranium-238 $\left({ }^{238} \mathrm{U}\right)$, and plutonium-239 $\left({ }^{239} \mathrm{Pu}\right)$ at $\mathrm{U}$-ah/at and ${ }^{239} \mathrm{Pu},{ }^{222} \mathrm{Rn}$, and ${ }^{228} \mathrm{Ra}$ at U-3bh.

Table 16. Area 3 RWMS v2.102 GoldSim Model Acute Drilling Intruder TED

\begin{tabular}{|c|c|c|c|}
\hline Scenario/Disposal Unit & Mean TED $(\mathbf{m S v})$ & $\mathbf{9 5}^{\text {th }}$ Percentile $(\mathbf{m S v})$ & Time of Maximum \\
\hline Drilling/U-3ah/at & $2.3 \mathrm{E}-4$ & $4.5 \mathrm{E}-4^{\dagger}$ & 1,000 years \\
\hline Drilling/U-3bh & $4.2 \mathrm{E}-4$ & $1.2 \mathrm{E}-3$ & 1,000 years \\
\hline
\end{tabular}

${ }^{\dagger}$ - Maximum $95^{\text {th }}$ percentile value occurs at 140 years

The acute construction intruder TED is greater than the drilling intruder results. The mean and $95^{\text {th }}$ percentile acute construction intruder TEDs are a small fraction of the $5 \mathrm{mSv}$ performance objective for both post-1988 disposal units (Table 17). The acute construction TED is due predominantly to ${ }^{239} \mathrm{Pu},{ }^{222} \mathrm{Rn}$, and ${ }^{238} \mathrm{U}$ at $\mathrm{U}$-ah/at and ${ }^{222} \mathrm{Rn},{ }^{228} \mathrm{Th},{ }^{239} \mathrm{Pu}$, and ${ }^{228} \mathrm{Ra}$ at U-3bh.

Table 17. Area 3 RWMS v2.102 GoldSim Model Acute Construction Intruder TED

\begin{tabular}{|c|c|c|c|}
\hline Scenario/Disposal Unit & Mean TED (mSv) & 95 $^{\text {th }}$ Percentile (mSv) & Time of Maximum \\
\hline Construction/U-3ah/at & 0.014 & 0.036 & 1,000 years \\
\hline Construction/U-3bh & 0.016 & 0.037 & 1,000 years \\
\hline
\end{tabular}

The special analysis results indicate that changes to the Area 3 RWMS PA model do not significantly alter the PA results or conclusions. Although increases occur for the all-pathways annual TED and the ${ }^{222} \mathrm{Rn}$ flux density, all PA results are a small fraction of the performance objectives. The same conclusion was reached for the approved PA. The special analysis results continue to support a conclusion that there is a reasonable expectation of meeting all performance objectives. There is no need to revise the DAS at this time. The special analysis results support a conclusion that the Area 3 RWMS PAs remain valid, and revision is not necessary at this time.

\subsubsection{Area 5 RWMS}

A new baseline version of the Area 5 RWMS PA model, version 4.114, was released in FY 2012. The performance of the Area 5 RWMS was analyzed using the Area 5 RWMS v4.114 GoldSim model to assess the continuing validity of PA conclusions. The geometric mean 
inventory and standard deviation data listed in Tables 9 through 11 were entered into the inventory elements for the SLB, Pit 6, Pit 13 disposal units, and GCD boreholes, respectively. The disposal unit area, disposal unit volume, and waste volumes were updated with FY 2012 data. All SLB disposal units were assumed to be closed with a $2.5 \mathrm{~m}(8.2 \mathrm{ft})$ thick cover. The model was run assuming a median period of active institutional control of 245 years, a 100-year period of passive institutional control, and a 1,000-year compliance period. The model was run in GoldSim version 10.5(SP3) with 5,000 LHS realizations.

The results for the Area 5 RWMS v4.114 model indicate that there is reasonable expectation of compliance with the member of public performance objectives. The atmospheric pathway mean and $95^{\text {th }}$ percentile annual TED for all scenarios are less than the limit of $0.1 \mathrm{mSv}$ (Table 18). The air pathways results were largely unchanged in FY 2012. The maximum air pathway annual TED is less than $1 \%$ of the performance objective. The peak annual TED occurs at 1,000 years for all scenarios. The predominant source of atmospheric pathway dose in FY 2012 was ${ }^{238} \mathrm{U}$ and ${ }^{229}$ Th for all scenarios with peak TED at 1,000 years.

Table 18. Area 5 RWMS v4.114 GoldSim Model Member of Public Total Annual TED through the Air Pathway

\begin{tabular}{|c|c|c|c|}
\hline Exposure Scenario & Mean $\mathbf{( m S v )}$ & $\mathbf{9 5}^{\text {th }}$ Percentile $\mathbf{( m S v )}$ & $\begin{array}{c}\text { Time of } \\
\text { Maximum }\end{array}$ \\
\hline Transient Visitor & $8.2 \mathrm{e}-05$ & $3.0 \mathrm{E}-04$ & 1,000 years \\
\hline Resident & $1.6 \mathrm{E}-04$ & $5.5 \mathrm{E}-04$ & 1,000 years \\
\hline Resident Farmer & $4.5 \mathrm{E}-04$ & $1.6 \mathrm{E}-03$ & 1,000 years \\
\hline Open Rangeland (Cane Spring) & $6.6 \mathrm{E}-09$ & $1.8 \mathrm{E}-08$ & 1,000 years \\
\hline Open Rangeland (NNSS Boundary) & $1.1 \mathrm{E}-07$ & $3.0 \mathrm{E}-07$ & 1,000 years \\
\hline
\end{tabular}

The mean and $95^{\text {th }}$ percentile annual TEDs for the all-pathways scenarios are less than the $0.25 \mathrm{mSv}$ performance objective (Table 19). The all-pathway TEDs show slight increases for all scenarios, except the open rangeland scenarios, which decrease slightly. The maximum all-pathway TED increases 1\%, reaching approximately $8 \%$ of the performance objective.

Table 19. Area 5 RWMS v4.114 GoldSim Model Member of Public Annual TED through All Pathways

\begin{tabular}{|c|c|c|c|}
\hline Exposure Scenario & Mean $\mathbf{( m S v )}$ & $\mathbf{9 5}^{\text {th }}$ Percentile $(\mathbf{m S v})$ & $\begin{array}{c}\text { Time of } \\
\text { Maximum }\end{array}$ \\
\hline Transient Visitor & $6.5 \mathrm{E}-03$ & $1.6 \mathrm{E}-02$ & 1,000 years \\
\hline Resident & $8.3 \mathrm{E}-04$ & $2.6 \mathrm{E}-03$ & 1,000 years \\
\hline Resident Farmer & $2.1 \mathrm{E}-02$ & $6.8 \mathrm{E}-02$ & 1,000 years \\
\hline Open Rangeland (Cane Spring) & $3.1 \mathrm{E}-03$ & NA & 100 years \\
\hline Open Rangeland (NNSS Boundary) & $3.3 \mathrm{E}-03$ & NA & 100 years \\
\hline
\end{tabular}

NA - not available, insufficient realizations to calculate $95^{\text {th }}$ percentile 
The transient visitor scenario all-pathways annual TED is dominated by external exposure to

${ }^{222} \mathrm{Rn}$ progeny in cover soil. The resident scenario TED is due predominantly to ${ }^{238} \mathrm{U},{ }^{210} \mathrm{~Pb}$, and

${ }^{229} \mathrm{Th}$. The other scenarios all include agriculture and are dominated by ingestion of ${ }^{99} \mathrm{Tc}$ at 1,000 years or ${ }^{3} \mathrm{H}$ at 100 years.

The mean and $95^{\text {th }}$ percentile ${ }^{222} \mathrm{Rn}$ flux densities are less than the $0.74 \mathrm{~Bq} \mathrm{~m}^{-2} \mathrm{~s}^{-1}$ performance objective averaged over the entire site (Table 20). The same is true for all virtual disposal units, except for the Pit $13 \mathrm{RaDU}$, where the $95^{\text {th }}$ percentile ${ }^{222} \mathrm{Rn}$ flux density exceeds the performance objective. The flux density result for the Pit $13 \mathrm{RaDU}$ is not considered significant, because the performance objective is compared with the flux averaged over the site, not the flux from a portion of an individual disposal unit. Results for the Pit 6 RaDU, the Pit 13 RaDU, and GCD are essentially unchanged in FY 2012.

Table 20. Area 5 RWMS v4.114 GoldSim Model ${ }^{222}$ Rn Flux Density Results

\begin{tabular}{|c|c|c|c|}
\hline Disposal Unit & Mean $\left(\mathbf{B q ~} \mathbf{~}^{-\mathbf{2}} \mathbf{~}^{\mathbf{- 1}}\right)$ & $\mathbf{9 5}^{\text {th }}$ Percentile $\left(\mathbf{B q ~} \mathbf{~}^{-\mathbf{2}} \mathbf{s}^{-\mathbf{1}}\right)$ & Time of Maximum \\
\hline All & 0.22 & 0.50 & 1,000 years \\
\hline SLB & 0.22 & 0.59 & 5 years \\
\hline Pit 6 RaDU & 0.080 & 0.16 & 1,000 years \\
\hline Pit 13 RaDU & 0.60 & 1.7 & 1,000 years \\
\hline GCD & $1.1 \mathrm{E}-08$ & $2.8 \mathrm{E}-08$ & 1,000 years \\
\hline
\end{tabular}

The ${ }^{222}$ Rn flux density averaged over all post-1988 Area 5 RMWS disposal units increased 37\% from FY 2011 to FY 2012. The ${ }^{222}$ Rn flux density averaged over all disposal units is $30 \%$ of the performance objective. The increase is due to additional ${ }^{226} \mathrm{Ra}$ inventory disposed in the SLB disposal units. Disposal of ${ }^{226} \mathrm{Ra}$ also changes the time history for the ${ }^{222} \mathrm{Rn}$ flux density. Previous results indicated low flux densities at closure rising to a maximum at the end of the compliance period. The FY 2012 inventory estimate produces a ${ }^{222} \mathrm{Rn}$ flux density that is relatively constant throughout the compliance period.

Based on the institutional control policy adopted in FY 2008, chronic intrusion is assumed to be an unlikely event. Chronic intrusion results are replaced with drilling and construction acute intruder scenario results. The mean and $95^{\text {th }}$ percentile acute intruder doses are less than the $5 \mathrm{mSv}$ performance measure for both scenarios at all virtual disposal units (Tables 21 and 22). The acute drilling scenario TEDs are not significantly different in FY 2012. The acute drilling intrusion TEDs remain a small fraction of the dose limit.

Table 21. Area 5 RWMS v4.114 GoldSim Model Acute Drilling Intruder TED

\begin{tabular}{|c|c|c|c|}
\hline Disposal Unit & Mean $(\mathbf{m S v})$ & $\mathbf{9 5}^{\text {th }}$ Percentile $(\mathbf{m S v})$ & Time of Maximum \\
\hline SLB & $1.6 \mathrm{E}-3$ & $2.8 \mathrm{E}-3$ & 1,000 years \\
\hline Pit 6 RaDU & 0.039 & 0.076 & 1,000 years \\
\hline Pit 13 RaDU & 0.026 & 0.033 & 1,000 years \\
\hline GCD & 0.017 & 0.043 & 1,000 years \\
\hline
\end{tabular}


The SLB disposal unit acute construction TEDs are largely unchanged in FY 2012. The mean and $95^{\text {th }}$ percentile are less than the performance objective for all scenarios. The mean SLB acute construction scenario TED is $24 \%$ of the dose limit.

Table 22. Area 5 RWMS v4.114 GoldSim Model Acute Construction Intruder TED

\begin{tabular}{|c|c|c|c|}
\hline Disposal Unit & Mean $(\mathbf{m S v})$ & $\mathbf{9 5}^{\text {th }}$ Percentile $(\mathbf{m S v})$ & Time of Maximum \\
\hline SLB & 1.2 & 2.2 & 1,000 years \\
\hline Pit 6 RaDU & 0.88 & 2.2 & 1,000 years \\
\hline Pit 13 RaDU & 0.053 & 0.16 & 1,000 years \\
\hline GCD & $3.2 \mathrm{E}-06$ & NA & 100 years \\
\hline
\end{tabular}

$\mathrm{NA}-$ not available, insufficient realizations to calculate $95^{\text {th }}$ percentile

The FY 2012 PA results show little or no change, except for the ${ }^{222} \mathrm{Rn}$ flux density, which increased. All results indicate that there is still reasonable assurance of meeting all performance objectives. Therefore, the Area 5 RWMS PA results are still considered valid, and no need to revise the PA is identified.

Comparison of the FY 2012 results with the 2006 PA update indicates that significant changes have occurred in the maximum TEDs and their time of occurrence. The air pathway results have increased for all scenarios, except the open rangeland scenario, and the time of the maximum TED shifted from 63 to 1,000 years. Although changes have occurred, the maximum air pathway TED is less than $1 \%$ of the performance objective. The all-pathways results have increased for the transient visitor but decrease for the other scenarios. The ${ }^{222} \mathrm{Rn}$ flux density has increased for all disposal units. The intruder scenarios analyzed have changed from chronic scenarios to acute scenarios. The changes occurring since the 2006 PA update reflect the cumulative effects of inventory changes, updated parameters, a new passive institutional control period, a new institutional control policy, a thinner closure cover, and new dose conversion factors.

\subsection{CONCLUSIONS}

\subsubsection{Area 3 RWMS}

The most significant change at the Area 3 RWMS is the increased inventory since the approved PA in 1996 and its placement in inactive status. The site's conceptual model, important FEPs, site characteristics, and compliance points remain unchanged. Environmental monitoring results continue to indicate that the only releases from the site are low levels of tritiated water that remain consistent with PA model results. Monitoring and R\&D results continue to confirm and support the hydrologic conceptual model.

The three key questions can be answered as follows:

1. Does the annual summary information indicate that changes to the PA are required? A special analysis of the Area 3 RWMS (NSTec 2012d) demonstrates that PA conclusions are unchanged and that there continues to be a reasonable expectation of compliance with all performance objectives. A full PA revision is not necessary at this time. 
2. Does the annual summary information indicate that the conclusions of the PA remain valid? The special analysis results confirm that important PA conclusions remain unchanged. No groundwater pathway is expected at the site. All PA results continue to be a small fraction of their performance objectives.

3. Does the annual summary information indicate that facility performance will remain within the DOE M 435.1-1 PA performance objectives and any conditions in the facility DAS? The FY 2012 special analysis results indicate that there is still a reasonable expectation of compliance with all performance objectives.

\subsubsection{Area 5 RWMS}

The most significant changes for the Area 5 RWMS since preparation of the 2006 PA update include increased inventory, updated parameters, revised periods of institutional control, and a thinner closure cover. The conceptual model, important FEPs, site characterization data, and compliance points remain unchanged. Therefore, no new revision to the Area 5 RWMS PA is necessary.

The three key questions can be answered as follows:

1. Does the annual summary information indicate that changes to the PA or CA are required? A revision of the Area 5 RWMS PA is not necessary at this time.

2. Does the annual summary information indicate that the conclusions of the PA and CA remain valid? Although a number of changes have occurred since preparation of the 2006 PA update, the PA's conclusions continue to remain valid.

3. Does the annual summary information indicate that facility performance will remain within the DOE M 435.1-1 PA performance objectives and any conditions in the facility DAS? Updated FY 2012 PA results indicate that there is still a reasonable expectation of compliance with all performance objectives. 
This Page Intentionally Left Blank 


\subsection{COMPOSITE ANALYSIS REVIEW}

The CA evaluates the combined impacts of radionuclide releases from LLW disposal facilities and all other interacting sources of radioactive materials. The PA review above summarizes changes relevant to waste disposed after September 26, 1988. The CA review emphasizes changes not addressed in the PA review. CA source terms not addressed in the PA review include the pre-1988 RWMS waste inventory and residual radioactive materials from Environmental Restoration (ER) sites that interact with the RWMSs. Radioactively contaminated ER sites are mostly surface soils and groundwater units contaminated by nuclear weapons testing. The pre-1988 disposal units at the Area 3 RWMS and Area 5 RWMS are the only facilities that interact with the Area 3 and Area 5 RWMSs. Discussion of facility changes in the CA review is limited to changes at the pre-1988 RWMS disposal units.

\subsection{WASTE OPERATIONS AND ENVIRONMENTAL REMEDIATION}

\subsubsection{Radioactive Waste Management Sites}

\subsubsection{Waste Characteristics and Facility Design}

There were no discovered or proposed changes in the operation, facility design, and waste characteristics of the pre-1988 disposal units at the Area 3 and Area 5 RWMS. All pre-1988 disposal units at the Area 3 RWMS and Area 5 RWMS are now closed, except for the U-3ah/at disposal unit at the Area 3 RWMS, which is operationally closed. No operational changes occurred at either site. The CA models remain consistent with the facility designs and waste characteristics.

No new information is available concerning pre-1988 waste forms, containers, and operations at the Area 3 RWMS. No remediation of pre-1988 wastes or disposal units was performed. A special analysis for the Area 3 RWMS pre-1988 waste was released in FY 2012. Revised Area 3 RWMS disposal unit volume estimates were prepared to support the special analysis. There were no significant changes to the pre-1988 waste inventories for the Area 3 RWMS. The current Area 3 RWMS CA inventory was estimated with the Area 3 Inventory v2.016 model.

Pre-1988 waste forms, containers, facility design, and operations at the Area 5 RWMS are unchanged in FY 2012. No remediation involving pre-1988 wastes or disposal units was performed. No special analyses relevant to the Area 5 RWMS pre-1988 wastes were performed. There were no significant changes to the pre-1988 waste inventories for the Area 5 RWMS. The current Area 5 RWMS CA inventory was estimated with the Area 5 Inventory v2.110 model.

\subsubsection{Monitoring}

Pre-1988 waste and disposal units are subject to the monitoring activities discussed in Section 2.2.3. The results of environmental monitoring across the NNSS are reported annually in the Annual Site Environmental Report and the National Emission Standards for Hazardous Air Pollutants report (NSTec 2012b, 2012c). CY 2011 monitoring results are consistent with previous results and the CA resuspension and atmospheric dispersion model results. No 
significant subsidence events were observed at pre-1988 disposal units at the Area 3 and Area 5 RWMS in CY 2011.

\subsubsection{Closure}

The Area 3 RWMS PA/CA assumes that the site will be closed with a vegetated monolithic ET

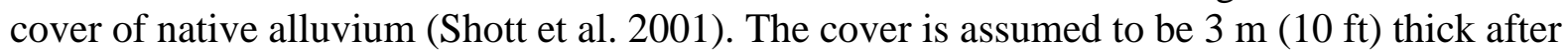
subsidence. The U-3ax/bl disposal unit, which contains most of the pre-1988 at the Area 3 RWMS, was closed in FY 2001 with the installation of a monolithic alluvium cover. The existing U-3ax/bl $2.7 \mathrm{~m}$ (8.9 ft) operational cover was supplemented with an additional $0.3 \mathrm{~m}$ $(1 \mathrm{ft})$ of soil and sloped to promote drainage off the cover. The U-3ax/bl closure cover is consistent with the CA assumption of a $3 \mathrm{~m}$ (10 ft) monolithic cover. Current plans are to close U-3ah/at and U-3bh with a 3 m (10 ft) monolithic ET cover (NSTec 2007b). The Area 3 RWMS covers and closure plan remain consistent with CA model assumptions.

The Area 5 RWMS CA cover assumptions are consistent with closure plans (BN 2001b; NSTec 2008a). The CA assumes that the cover is maintained for 100 years and public access is restricted for 250 years. The cover is assumed to be a monolithic ET cover, measuring 2 to $6 \mathrm{~m}$ (6 to $20 \mathrm{ft}$ ) thick. The current Area 5 RWMS CA model assumes the site is closed with a $2.5 \mathrm{~m}$ (8 ft) monolithic ET cover. In FY 2011, the 92-ac LLWMU at the Area 5 RWMS, which includes all pre-1988 disposal units except the GCD Test borehole, was closed with a $2.5 \mathrm{~m} \mathrm{(8 \textrm {ft } )}$ monolithic ET cover. Closure of the GCD and GCD Test boreholes will occur at final site closure in 2028. Closure of the pre-1988 Area 5 RWMS disposal units and the closure plan are consistent with the CA assumptions (NSTec 2008a).

\subsection{RESEARCH AND DEVELOPMENT}

No R\&D activities specific to pre-1988 waste or residual radioactive contamination had results that might impact the CA results and conclusions in FY 2012. The model development R\&D activities described in Section 2.4 are also relevant for the CAs.

\subsection{INTERACTING SOURCE TERMS}

\subsubsection{Underground Test Areas}

The goal of UGTA CAU closure under the FFACO process is to establish groundwater regulatory boundaries with corresponding use restriction boundaries. Negotiation of CAU regulatory boundaries will proceed through a CADD/CAP stage and a Closure Report stage. An initial use restriction boundary and regulatory boundary objectives are identified at the start of the CADD/CAP stage. The use restriction boundary will be finalized and the regulatory boundary will be established at the start of the Closure Report stage of the UGTA strategy. The use restriction boundary is established through combined assessments of contaminant boundary forecasts, requirements for protection of worker health and safety, and administrative policies designed to restrict access to contaminated groundwater. A regulatory boundary is chosen to provide protection for the public and the environment from the effects of migration of radioactive contaminants. 
The Area 3 RWMS and Area 5 RWMS CAs assume that the sites are within the UGTA use restriction boundaries and that the use restrictions can control exposure of the public to groundwater contamination. In FY 2008, NNSA/NSO implemented a formal policy to implement and maintain the UGTA use restrictions (NNSA/NSO 2008).

The Yucca Flat CAU 97 is still in the corrective action investigation stage of the FFACO process. An internal preemptive review of the draft CAU 97 groundwater flow and radionuclide transport document for CAU 97 was completed in FY 2012. The Area 3 RMWS is expected to be within the initial use restriction boundary for CAU 97. The expected completion data of final flow and transport model results simulating alternative forecasts of the 1,000-year groundwater contaminant boundaries for Yucca Flat has moved from FY 2023 to FY 2015. This change may allow acceleration of completion of the Area 3 RWMS CA. The Area 3 RWMS CA assumptions are still consistent with current plans for Yucca Flat CAU 97.

Frenchman Flat CAU 98 is in a more advanced stage of the FFACO process. The Frenchman Flat CAU 98 CADD/CAP was completed and accepted by NDEP in 2011 (NNSA/NSO 2011b). The CADD portion describes the results of the CAU 98 data collection and modeling activities; the CAP portion describes the corrective action implementation plan. The CAP also identifies negotiated CAU regulatory boundary objectives and the initial use restriction boundaries. Drilling activities supporting model evaluation studies identified in the CADD/CAP were initiated in FY 2012. If the evaluations and any model refinements are accepted by NDEP, the final use restriction boundaries for Frenchman Flat will be negotiated at the start of the Closure Report stage in 2015. Consistent with the CA assumptions, the Area 5 RWMS is currently within the CAU 98 use restriction boundary. The Area 5 RWMS CA will require revision after final closure of Frenchman Flat CAU 98 scheduled for FY 2015.

\subsubsection{Soil Sites}

The CAs included multiple contaminated Soil Sites characterized by the Radionuclide Inventory and Distribution Program (RIDP) (McArthur 1991) as source terms for atmospheric resuspension and dispersion modeling. The CAs assume that the contaminated Soil Sites will be closed in place and any corrective actions will have minimal impacts on resuspension and atmospheric dispersion from the Soil Sites to the RWMSs.

The Area 3 RWMS CA included 28 Soil Sites contaminated by aboveground and belowground nuclear testing. Many of these sites have progressed in the FFACO process since preparation of the Area 3 RWMS CA (Table 23). Additional site characterization data and closure activities continue to be consistent with the Area 3 RWMS CA assumptions. Completed or planned closure actions have had no significant impact on radionuclide inventories or radionuclide resuspension rates. Completed and planned corrective actions are not expected to have any impact on the CA model assumptions. 
Table 23. Status of ER Soil Sites Considered in the Area 3 RWMS CA

\begin{tabular}{|c|c|c|c|c|}
\hline CAU & Site & $\begin{array}{l}\text { Radiological } \\
\text { COPCs or } \\
\text { COCs }\end{array}$ & FFACO Status & Corrective Action \\
\hline 104 & $\begin{array}{c}\text { Area } 7 \text { Atmospheric } \\
\text { Test Sites }\end{array}$ & None & $\begin{array}{l}\text { CADD/CAP Issued } \\
\text { (NNSA/NSO 2012d) }\end{array}$ & $\begin{array}{c}\text { Remove Hazardous PSM, } \\
\text { No Further Action } \\
\text { (Test Site B7-B Clean Closure) }\end{array}$ \\
\hline 105 & $\begin{array}{c}\text { Area } 2 \text { Atmospheric } \\
\text { Test Sites }\end{array}$ & $\begin{array}{c}\text { MFP, } \\
\text { Actinides }\end{array}$ & $\begin{array}{c}\text { CAIP Issued } \\
\text { (NNSA/NSO 2012e) }\end{array}$ & TBD \\
\hline \multirow{2}{*}{107} & $\begin{array}{l}13 \text { Low Impact Soil } \\
\text { Sites }\end{array}$ & None & \multirow{2}{*}{$\begin{array}{c}\text { CR Issued } \\
\text { (NNSA/NSO 2009b) }\end{array}$} & No Action \\
\hline & $\begin{array}{l}\text { SULKY, TRUCHAS- } \\
\text { CHAMISAL }\end{array}$ & Radionuclides & & $\begin{array}{c}\text { Closure in Place with } \\
\text { Administrative Controls }\end{array}$ \\
\hline 365 & $\begin{array}{c}\text { BANEBERRY } \\
\text { Contamination Area }\end{array}$ & Radionuclides & $\begin{array}{l}\text { CADD/CR Issued } \\
\text { (NNSA/NSO 2011c) }\end{array}$ & $\begin{array}{l}\text { Closure in Place with Use } \\
\text { Restrictions }\end{array}$ \\
\hline 367 & $\begin{array}{l}\text { Area } 10 \text { SEDAN, } \\
\text { ESS, and UNCLE } \\
\text { Craters }\end{array}$ & Radionuclides & $\begin{array}{l}\text { CADD/CR Issued } \\
\text { (NNSA/NSO 2011d) }\end{array}$ & $\begin{array}{c}\text { Closure in Place with Use } \\
\text { Restrictions }\end{array}$ \\
\hline 370 & $\begin{array}{l}\text { T-4 Atmospheric Test } \\
\text { Site }\end{array}$ & Radionuclides & $\begin{array}{l}\text { CADD/CR Issued } \\
\text { (NNSA/NSO 2009c) }\end{array}$ & $\begin{array}{l}\text { Closure in Place with Use } \\
\text { Restrictions }\end{array}$ \\
\hline 550 & $\begin{array}{c}\text { SMOKEY } \\
\text { Contamination Area }\end{array}$ & $\begin{array}{c}\text { MFP, } \\
\text { Actinides }\end{array}$ & $\begin{array}{c}\text { CAIP Issued } \\
\text { (NNSA/NSO 2012f) }\end{array}$ & TBD \\
\hline 568 & $\begin{array}{l}\text { Area } 3 \text { Plutonium } \\
\text { Dispersal Sites }\end{array}$ & $\begin{array}{c}\text { MFP, } \\
\text { Actinides }\end{array}$ & $\begin{array}{c}\text { Preliminary } \\
\text { Investigations Initiated }\end{array}$ & TBD \\
\hline 569 & $\begin{array}{c}\text { Area } 3 \text { Yucca Flat } \\
\text { Atmospheric Test } \\
\text { Sites }\end{array}$ & $\begin{array}{c}\text { MFP, } \\
\text { Actinides }\end{array}$ & $\begin{array}{c}\text { CAIP Issued } \\
\text { (NNSA/NSO 2012g) }\end{array}$ & TBD \\
\hline 570 & $\begin{array}{c}\text { Area } 9 \text { Yucca Flat } \\
\text { Atmospheric Test } \\
\text { Sites }\end{array}$ & $\begin{array}{c}\text { MFP, } \\
\text { Actinides }\end{array}$ & $\begin{array}{c}\text { CAIP Issued } \\
\text { (NNSA/NSO 2012h) }\end{array}$ & TBD \\
\hline
\end{tabular}

CADD - Corrective Action Decision Document

CAP - Corrective Action Plan

CAIP - Corrective Action Investigation Plan

COCs - contaminants of concern

COPCs - contaminants of potential concern

CR - Closure Report

MFP - mixed fission products

PSM - potential source material

TBD - to be determined

The Area 3 RWMS special analysis includes one additional atmospheric testing Soil Site, the unsurveyed region of Area 2, which was not included in the CA. The omission was likely an oversight. The Area 3 RWMS special analysis results confirm that the Area 2 unsurveyed region is a minor source that has no significant impact on the CA results.

The Area 5 RWMS CA considered eight contaminated Soil Site CAUs as possible sources of residual contamination. Four of the Soil Sites, the 306 Ground Zero (GZ) Rad Contaminated Area (Corrective Action Site [CAS] 05-45-04), the 307 GZ Rad Contaminated Area (CAS 05-45-05), 
the Kay Blockhouse (CAS 05-33-01), and the Gravel Gertie (CAS 05-23-01) were excluded from the CA based on their small inventories, small area, and distance from the RWMS relative to other potential sources. The Pu Valley soil site (CAU 366) was excluded based on the assumption that the intervening mountain ranges blocked atmospheric dispersion. Only three sources, the Gadget, Mechanics, and Explosives (GMX) site (CAS 05-23-15), PINSTRIPE (CAS 11-23-05), and the Frenchman Flat Atmospheric Test Site (ABLE CAS 05-23-05, HAMILTON CAU 573, and SMALL BOY CAU 541), were explicitly included in CA modeling.

Most of the contaminated Soil Sites have advanced in the FFACO process since preparation of the Area 5 RWMS CA (Table 24). Among the four sites excluded from the Area 5 RWMS CA, additional site characterization confirms that these sources have small areas and inventories. Six of the eight sources currently have no radiological contaminants of concern (COCs) in surface soils or will have no COCs after final remediation. Radiological COCs are defined as radionuclides capable of causing an annual TED of $0.25 \mathrm{mSv}$ or more in one of three possible scenarios: industrial use, remote work, or occasional use. Although corrective action investigations have developed additional site characterization data, the RIDP characterization is still the preferred data source for contaminated Soil Sites inventories (NSTec 2008b). Completed or planned closure actions have had no significant impact on radionuclide inventories or radionuclide resuspension rates. CA assumptions remain consistent with the status of the Soil Sites. Completed and planned corrective actions are not expected to have any impact on CA model assumptions.

Table 24. Status of ER Soil Sites Considered in the Area 5 RWMS CA

\begin{tabular}{|c|c|c|c|c|}
\hline CAU & Site & $\begin{array}{l}\text { Radiological } \\
\text { COPCs or } \\
\text { COCs }\end{array}$ & $\begin{array}{l}\text { FFACO } \\
\text { Status }\end{array}$ & Corrective Action \\
\hline \multirow{3}{*}{106} & $\begin{array}{c}\text { ABLE Atmospheric } \\
\text { Test Site } \\
\text { (CAS 05-23-05) }\end{array}$ & None & \multirow{3}{*}{$\begin{array}{l}\text { CADD/CR Issued } \\
\text { (NNSA/NSO 2011e) }\end{array}$} & No Action \\
\hline & $\begin{array}{c}306 \text { GZ Rad } \\
\text { Contaminated Area } \\
\text { (CAS 05-45-04) }\end{array}$ & $\begin{array}{c}\text { None after } \\
\text { PSM Removal }\end{array}$ & & Remove PSM (DU) \\
\hline & $\begin{array}{c}307 \text { GZ Rad } \\
\text { Contaminated Area } \\
\text { (CAS 05-45-05) } \\
\end{array}$ & $\begin{array}{c}\text { None after } \\
\text { PSM Removal }\end{array}$ & & Remove PSM (DU) \\
\hline 140 & $\begin{array}{l}\text { Gravel Gertie } \\
\text { (CAS 05-23-01) }\end{array}$ & $\begin{array}{l}\text { Uranium/DU in } \\
\text { Bunker }\end{array}$ & $\begin{array}{c}\text { CR Issued } \\
\text { (NNSA/NSO 2005) }\end{array}$ & $\begin{array}{l}\text { Closure in Place with } \\
\text { Administrative Controls }\end{array}$ \\
\hline 204 & $\begin{array}{l}\text { Kay Blockhouse } \\
\text { (CAS 05-33-01) }\end{array}$ & DU & $\begin{array}{c}\text { CADD Issued } \\
\text { (NNSA/NSO 2004) }\end{array}$ & $\begin{array}{l}\text { Preferred Alternative - } \\
\text { Removal of Contaminated Soil } \\
\text { and Debris; Closure in Place } \\
\text { with Administrative Controls }\end{array}$ \\
\hline 366 & Plutonium Valley & $\begin{array}{l}{ }^{238} \mathrm{Pu},{ }^{239} \mathrm{Pu}, \\
{ }^{240} \mathrm{Pu},{ }^{241} \mathrm{Pu}, \\
{ }^{441} \mathrm{Am}\end{array}$ & $\begin{array}{c}\text { CADD Issued } \\
\text { (NNSA/NSO 2012c) }\end{array}$ & $\begin{array}{c}\text { Preferred Alternative - Closure } \\
\text { in Place with Administrative } \\
\text { Controls }\end{array}$ \\
\hline
\end{tabular}




\begin{tabular}{|c|c|c|c|c|}
\hline CAU & Site & $\begin{array}{l}\text { Radiological } \\
\text { COPCs or } \\
\text { COCs }\end{array}$ & $\begin{array}{l}\text { FFACO } \\
\text { Status }\end{array}$ & Corrective Action \\
\hline 371 & $\begin{array}{l}\text { PINSTRIPE } \\
\text { (CAS 11-23-05) }\end{array}$ & $\begin{array}{c}\text { None, } \\
\text { Subsurface } \\
\text { Contamination } \\
\text { Likely Present }\end{array}$ & $\begin{array}{l}\text { CADD/CR Issued } \\
\text { (NNSA/NSO 2010) }\end{array}$ & $\begin{array}{l}\text { Closure in Place with } \\
\text { Administrative Controls }\end{array}$ \\
\hline 541 & SMALL BOY & $\begin{array}{c}\text { MFP, } \\
\text { Actinides }\end{array}$ & No Action & TBD \\
\hline \multirow{2}{*}{573} & $\begin{array}{c}\text { GMX } \\
\text { (CAS 05-23-15) }\end{array}$ & ${ }^{239} \mathrm{Pu}$ & No Action & TBD \\
\hline & HAMILTON & $\begin{array}{c}\text { MFP, } \\
\text { Actinides }\end{array}$ & No Action & TBD \\
\hline
\end{tabular}

CADD - Correction Action Decision Document

COPC - contaminant of potential concern

COC - contaminant of concern

CR - Closure Report

DU - depleted uranium

GMX - Gadget, Mechanics, and Explosives

MFP - mixed fission products

PSM - potential source material

TBD - to be determined

\subsubsection{Industrial Sites}

The CAs assume that the impact of the Industrial Sites is insignificant compared with the Soil Sites. No Industrial Sites are included in the CAs.

From FY 2007 to FY 2009 several Industrial Sites within CAU 547, Miscellaneous Contaminated Waste Sites, were discovered to have significant TRU inventories (NNSA/NSO 2011f). CAS 3-99-19 within CAU 547 is a gas sampling assembly associated with the TEJON/BERNALILLO events, located in Area 3 approximately 350 m (1,150 ft) west-northwest of the RWMS boundary. The CAS 3-99-19 $95^{\text {th }}$ percentile ${ }^{239+240} \mathrm{Pu}$ inventory, 3.7E11 Bq, is contained in steel pipes, much of it below an earthen berm (NNSA/NSO 2012i).

The CAS 3-99-19 ${ }^{239+240} \mathrm{Pu}$ inventory is of the same order of magnitude as the HORNET GZ contaminated Soil Site at the Area 3 RWMS boundary. The HORNET GZ contaminated Soil Site was characterized by soil sampling and in situ gamma spectrometry. Consequently, the HORNET GZ contaminated Soil Site inventory most likely does not include the CAS 3-99-19 ${ }^{239+240} \mathrm{Pu}$ inventory. Therefore, the CAS 3-99-19 ${ }^{239+240} \mathrm{Pu}$ inventory is potentially a discovered inventory not included in the Area 3 RWMS CA inventory.

In 2012, the CAS 3-99-19 site was closed and the Closure Report issued (NNSA/NSO 2012i). The site was closed in place with a packed earthen berm and administrative controls.

Radionuclide releases and doses are projected to be low because the source term is contained in a steel pipe that is below a 0.6-1.2 m (2-4 ft) thick earthen berm. CAS 3-99-19 was excluded from the Area 3 RWMS special analysis based on the low dose estimate for the site (NSTec 2012d). 
No Industrial Sites have been characterized or remediated that impact interacting radiological sources in Frenchman Flat since preparation of the Area 5 RWMS CA. The Area 5 RWMS CA assumptions remain unchanged.

\subsection{SUMMARY OF CHANGES}

\subsubsection{Discovered Changes}

Two discovered changes are reported for the Area 3 RWMS CA. An industrial site, CAU 547, with a CAS located near the Area 3 RWMS was discovered to have a potentially significant plutonium inventory. The source, which is contained in a steel pipe and closed with a soil cover, was found to be an insignificant source for the CA. The CAU 547 source was not included in the Area 3 RWMS special analysis. One additional atmospheric testing Soil Site, the Area 2 unsurveyed region, was included in the Area 3 RWMS special analysis. Addition of the Area 2 unsurveyed region Soil Site to the CA had no significant impact on CA results.

The Area 5 RWMS CA had no discovered changes in FY 2012.

\subsubsection{Proposed Changes}

The Area 3 RWMS has been inactive since FY 2006. Therefore, no significant operational changes occurred for the Area 3 RWMS. Closure of ER sources included in the CA is progressing, but closure activities have had no impact on the CA model assumptions. An updated Area 3 RWMS CA model was used for a special analysis in FY 2012. CA model changes are described in the special analysis (NSTec 2012d).

The Area 5 RWMS 92-ac LLWMU, which includes all pre-1988 waste disposal units, was closed with a $2.5 \mathrm{~m}$ (8.2 ft) monolithic ET cover in FY 2011. Revegetation of the 92-ac LLWMU cover began in FY 2012. Closure of ER sources included in the CA is progressing, but closure activities have had no impact on the CA models. Corrective action investigations at several ER sources excluded from the CA confirm assumptions that the sources have minimal potential to interact with the Area 5 RWMS. An updated Area 5 RWMS CA baseline model was released in FY 2012. There were no significant changes to the CA model.

The maintenance plan, closure plan, monitoring plan, and R\&D plan are unchanged from previous years. Results from monitoring and R\&D are consistent with previous results and continue to support CA conceptual models. No revision of the maintenance plan, closure plan, monitoring plan, or R\&D plan are required.

\subsubsection{R\&D Changes}

\subsubsection{CA Results for the Area 3 RWMS}

The Area 3 RWMS v2.102 GoldSim model was used to update the CA results in FY 2012 (NSTec 2012d). The model was run as described for the PA, except that the model was placed in CA mode.

The CA annual TED continues to be predominantly from inhalation of ${ }^{239} \mathrm{Pu}$ resuspended from the HORNET GZ Soil Site, which surrounds the Area 3 RWMS. The time of the maximum dose shifts 
from 250 years in the approved CA to 1,000 years in the special analysis. The shift in the timing of the maximum dose is a result of the probabilistic period of institutional control used in the special analysis. The CA annual TED also increases from $0.01 \mathrm{mSv}$ in the approved CA to $0.02 \mathrm{mSv}$ in the special analysis (Table 25). The increase is caused by updated parameter values in the soil resuspension model. The mean and $95^{\text {th }}$ percentile doses are significantly less than the $0.3 \mathrm{mSv}$ annual dose constraint, and the importance of the contributing sources is unchanged. Therefore, the Area 3 RWMS CA results are still considered valid.

Table 25. Area 3 RWMS v2.102 GoldSim Model CA All-Pathways Annual TED for a Resident at the $100 \mathrm{~m}$ (330 ft) RWMS boundary

\begin{tabular}{|c|c|c|c|}
\hline Disposal Unit & Mean $(\mathbf{m S v})$ & $\mathbf{9 5}^{\text {th }}$ Percentile $(\mathbf{m S v})$ & Time of Maximum \\
\hline All & 0.021 & 0.031 & 1,000 years \\
\hline
\end{tabular}

\subsubsection{CA Results for the Area 5 RWMS}

The Area 5 RWMS CA results were updated with the Area 5 RWMS v4.114 GoldSim model. The model was run as described for the PA, except that the model was placed in CA mode. A slight decrease is observed in FY 2012 for the dose at the Area 5 RWMS boundary (Table 26). The mean and $95^{\text {th }}$ percentile doses are significantly less than the $0.3 \mathrm{mSv}$ annual dose constraint. Therefore, the Area 5 RWMS CA results are still considered valid.

Table 26. Area 5 RWMS v4.114 GoldSim Model CA All-Pathways Annual TED for a Resident at the $100 \mathrm{~m}$ (330 ft) RWMS boundary

\begin{tabular}{|c|c|c|c|}
\hline Disposal Unit & Mean $(\mathbf{m S v})$ & $\mathbf{9 5}^{\text {th }}$ Percentile $(\mathbf{m S v})$ & Time of Maximum \\
\hline All & $9.9 \mathrm{E}-4$ & $3.0 \mathrm{E}-3$ & 1,000 years \\
\hline
\end{tabular}

\subsection{CONCLUSIONS}

\subsubsection{Area 3 RWMS}

There have been no changes in FY 2012 that affect the conclusions of the CA, as indicated by reviews of facility operations, the disposal unit closure inventories, inventories of the ER sources of residual radionuclides, the progress of the ER cleanup projects, land-use planning, closure planning, and the results of the monitoring and R\&D activities. Two new sources of contamination have been identified but were not found to have any significant impact on CA results. There is no new information that would reduce the uncertainty of the current sources. A special analysis of the Area 3 RWMS was issued in FY 2012, which includes updated CA doses.

The three key questions can be answered as follows:

1. Does the annual summary information indicate that changes to the PA or CA are required? A special analysis of the Area 3 RWMS CA was prepared to determine the impacts of changes occurring since preparation of the last CA. The special analysis concludes that a CA revision is not necessary at this time.

2. Does the annual summary information indicate that the conclusions of the PA and CA remain valid? The special analysis for the Area 3 RWMS CA indicates that the annual TED to a resident from all interacting sources is a small fraction of the $0.3 \mathrm{mSv}$ dose 
constraint and that the HORNET GZ Soil Site is the predominant source. The Area 3 RWMS special analysis results are consistent with the CA results, supporting a conclusion that the CA continues to be valid.

3. Does the annual summary information indicate that facility performance will remain within the DOE M 435.1-1 PA performance objectives, CA performance goals, and any conditions in the facility DAS? The Area 3 RWMS v2.102 GoldSim model results indicate that there is still a high likelihood of meeting of the $0.3 \mathrm{mSv}$ dose constraint.

\subsubsection{Area 5 RWMS}

There have been no changes in FY 2012 that affect the conclusions of the CA, as indicated by reviews of facility operations, the disposal unit closure inventories, estimated inventories of the ER sources of residual radionuclides, the progress of the ER corrective actions, land-use planning, closure planning, and the results of the monitoring and $R \& D$ activities. No new sources of contamination have been identified. Corrective action investigations at contaminated soil sites has confirmed and supported CA assumptions.

The only changes affecting the CA are the updated RWMS inventory estimates. The consequences of the changes were evaluated with the Area 5 RWMS v4.114 GoldSim model and found not to affect the CA conclusions.

The three key questions can be answered as follows:

1. Does the annual summary information indicate that changes to the PA or CA are required? A revision of the Area 5 RWMS CA is not necessary at this time.

2. Does the annual summary information indicate that the conclusions of the PA and CA remain valid? Review of the Area 5 RWMS CA indicates that the CA conclusions remain valid.

3. Does the annual summary information indicate that facility performance will remain within the DOE M 435.1-1 PA performance objectives, CA performance goals, and any conditions in the facility DAS? The Area 5 RWMS v4.114 GoldSim model results indicate that there is a high likelihood of meeting the $0.3 \mathrm{mSv}$ dose constraint. 
This Page Intentionally Left Blank 


\subsection{REFERENCES}

Bechtel Nevada, 2001a. Addendum 1, Performance Assessment for the Area 5 Radioactive Waste Management Site at the Nevada Test Site, Nye County, Nevada. DOE/NV/11718--176-ADD1. Las Vegas, NV. November 2001.

Bechtel Nevada, 2001b. Composite Analysis for the Area 5 Radioactive Waste Management Site at the Nevada Test Site, Nye County, Nevada. DOE/NV--594. Las Vegas, NV. September 2001.

Bechtel Nevada, 2001c. Addendum 1, Composite Analysis for the Area 5 Radioactive Waste Management Site at the Nevada Test Site, Nye County, Nevada. DOE/NV--594-ADD1. Las Vegas, NV. November 2001.

Bechtel Nevada, 2003. Nevada Test Site Routine Radiological Environmental Monitoring Plan. DOE/NV/11718--804. Las Vegas, NV. June 2003.

Bechtel Nevada, 2006. Addendum 2 to the Performance Assessment for the Area 5 Radioactive Waste Management Site at the Nevada Test Site, Nye County, Nevada. DOE/NV/11718--176-ADD2. Las Vegas, NV. January 2006.

BN, see Bechtel Nevada.

CFR, see Code of Federal Regulations.

Cochran, J. R., W. E. Beyeler, D. A. Brosseau, L. H. Bush, T. J. Brown, B. M. Crowe, S. H. Conrad, P. A. Davis, T. Ehrhorn, T. Feeney, B. Fogleman, D. P. Gallegos, R. Haaker, E. Kalinina, L. L. Price, D. P. Thomas, and S. Wirth, 2001. Compliance Assessment Document for the Transuranic Wastes in the Greater Confinement Disposal Boreholes at the Nevada Test Site, Volume 2: Performance Assessment. SAND2001-2977. Albuquerque, NM. September 2001.

Code of Federal Regulations, Title 40, Part 191, "Environmental Radiation Protection Standards for Management and Disposal of Spent Nuclear Fuel, High-Level, and Transuranic Radioactive Waste,” 1994.

DOE, see U.S. Department of Energy.

EPA, see U.S. Environmental Protection Agency.

McArthur, R. D., 1991. Radionuclides in Surface Soil at the Nevada Test Site. DOE/NV/10845--02. Water Resources Center, Desert Research Institute. Las Vegas, NV. August 1991. 
National Security Technologies, LLC, 2007a. Maintenance Plan for the Performance Assessments and Composite Analyses for the Area 3 and Area 5 Radioactive Waste Management Sites at the Nevada Test Site. DOE/NV/25946--091. Las Vegas, NV. January 2007.

National Security Technologies, LLC, 2007b. Closure Plan for the Area 3 Radioactive Waste Management Site at the Nevada Test Site. DOE/NV/25946--289. Las Vegas, NV. September 2007.

National Security Technologies, LLC, 2008a. Closure Plan for the Area 5 Radioactive Waste Management Site at the Nevada Test Site. DOE/NV/25946--553. Las Vegas, NV. September 2008.

National Security Technologies, LLC, 2008b. Radionuclide Inventory and Distribution Project Data Evaluation and Verification White Paper. DOE/NV/25946--994. Las Vegas, NV. December 2008.

National Security Technologies, LLC, 2011. 2010 Annual Summary Report for the Area 3 and Area 5 Radioactive Waste Management Sites at the Nevada Test Site, Nye County, Nevada. DOE/NV25946--893. Las Vegas, NV. March 2011.

National Security Technologies, LLC, 2012a. Nevada National Security Site Environmental Report 2011. DOE/NV25946--1604. Las Vegas, NV. September 2012.

National Security Technologies, LLC, 2012b. National Emission Standards for Hazardous Air Pollutants - Radionuclide Emissions Calendar Year 2011. DOE/NV/25946--1530. Las Vegas, NV. June 2012.

National Security Technologies, LLC, 2012c. Nevada National Security Site 2011 Waste Management Monitoring Report Area 3 and Area 5 Radioactive Waste Management Sites. DOE/NV/25946--1572. Las Vegas, NV. July 2012.

National Security Technologies, LLC, 2012d. Special Analysis of the Area 3 Radioactive Waste Management Site at the Nevada National Security Site, Nye County, Nevada. DOE/NV/25946--1617. Las Vegas, NV. September 2012.

National Security Technologies, LLC, 2013. Nevada National Security Site 2012 Data Report: Groundwater Monitoring Program, Area 5 Radioactive Waste Management Site. DOE/NV/25946--1683. Las Vegas, NV. February 2013.

NNSA/NSO, see U.S. Department of Energy, National Nuclear Security Administration Nevada Site Office.

NSTec, see National Security Technologies, LLC. 
Shott, G. J., L. E. Barker, S. E. Rawlinson, M. J. Sully, and B. A. Moore, 1998. Performance Assessment for the Area 5 Radioactive Waste Management Site at the Nevada Test Site, Nye County, Nevada, Rev. 2.1. DOE/NV/11718--176/UC-721. Bechtel Nevada, Las Vegas, NV. January 1998.

Shott, G. J., V. Yucel, M. J. Sully, L. E. Barker, S. E. Rawlinson, and B. A. Moore, 2001. Performance Assessment/Composite Analysis for the Area 3 Radioactive Waste Management Site at the Nevada Test Site, Nye County, Nevada, Rev. 2.1. DOE/NV--491-Rev 2.1. Bechtel Nevada, Las Vegas, NV. December 2001.

Shott, G. J., and V. Yucel, 2009. Optimization of the Area 5 Radioactive Waste Management Site Closure Cover. DOE/NV/25946--695. National Security Technologies, LLC, Las Vegas, NV. April 2009.

U.S. Department of Energy, 1999a. “Disposal Authorization Statement for the Department of Energy Nevada Operations Office Area 3 Radioactive Waste Management Site Low-Level Radioactive Waste Disposal Facility dated October 20, 1999.” Memorandum from EM-35 to Kathleen Carlson, Manager, Nevada Operations Office. October 21, 1999.

U.S. Department of Energy, 1999b. DOE M 435.1-1, “Radioactive Waste Management,” Washington, D.C., 1999.

U.S. Department of Energy, 1999c. DOE G 435.1-4, "Maintenance Guide for U.S. Department of Energy Low-Level Waste Disposal Facility Performance Assessments and Composite Analysis.” Washington, D.C., November 1999.

U.S. Department of Energy, 2000. "Disposal Authorization for the Nevada Test Site Area 5 Low-Level Waste Disposal Facility.” Memorandum from EM-35 to Kathleen Carlson, Manager, Nevada Operations Office. December 5, 2000.

U.S. Department of Energy, 2001. DOE O 435.1, "Radioactive Waste Management.” U.S. Department of Energy, Washington, D.C., 2001.

U.S. Department of Energy, 2002a. "Disposal Authorization for the Nevada Test Site Area 3 Radioactive Waste Management Site Submittal of Revised Performance Assessment and Composite Analysis dated December 17, 2001.” Memorandum from EM-43 to Kathleen Carlson, Manager, Nevada Operations Office. August 2, 2002.

U.S. Department of Energy, 2002b. "Disposal Authorization for the Nevada Test Site Area 5 Radioactive Waste Management Site Submittal of Revised Performance Assessment and Composite Analysis Addenda.” Memorandum from EM-43 to Kathleen Carlson, Manager, Nevada Operations Office. May 23, 2002.

U.S. Department of Energy, National Nuclear Security Administration Nevada Site Office, 2004. Corrective Action Decision Document for Corrective Action Unit 204: Storage Bunkers, Nevada Test Site, Nevada. DOE/NV--959. Las Vegas, NV. April 2004. 
U.S. Department of Energy, National Nuclear Security Administration Nevada Site Office, 2005. Closure Report for Corrective Action Unit 140: Waste Dumps, Burn Pits, and Storage Area, Nevada Test Site, Nevada. DOE/NV--1070. Las Vegas, NV. August 2005.

U.S. Department of Energy, National Nuclear Security Administration Nevada Site Office, 2006. Radioactive Waste Acceptance Program Implementation Plan. RWAP-P1, Rev. 4. Las Vegas, NV. March 30, 2006.

U.S. Department of Energy, National Nuclear Security Administration Nevada Site Office, 2007. Institutional Control Policies and Implementation for the Area 5 and Area 3 Radioactive Waste Management Sites. DOE/NV/25946--300. Las Vegas, NV. November 2007.

U.S. Department of Energy, National Nuclear Security Administration Nevada Site Office, 2008. Institutional Control of the Nevada Test Site. NSO P 454.X. Las Vegas, NV. January 8, 2008.

U.S. Department of Energy, National Nuclear Security Administration Nevada Site Office, 2009a. Corrective Action Decision Document/Corrective Action Plan for the 92-Acre Area and Corrective Action Unit 111: Area 5 WMD Retired Mixed Waste Pits, Nevada Test Site, Nevada. DOE/NV--1326. Las Vegas, NV. July 2009.

U.S. Department of Energy, National Nuclear Security Administration Nevada Site Office, 2009b. Closure Report for Corrective Action Unit 107: Low Impact Soil Sites, Nevada Test Site, Nevada. DOE/NV--1323. Las Vegas, NV. June 2009.

U.S. Department of Energy, National Nuclear Security Administration Nevada Site Office, 2009c. Corrective Action Decision Document/Closure Report for Corrective Action Unit 370: T-4 Atmospheric Test Site, Nevada Test Site, Nevada. DOE/NV--1320. Las Vegas, NV. May 2009.

U.S. Department of Energy, National Nuclear Security Administration Nevada Site Office, 2010. Corrective Action Decision Document/Closure Report for Corrective Action Unit 371: Johnnie Boy Crater and Pin Stripe, Nevada Test Site, Nevada. DOE/NV--1395. Las Vegas, NV. July 2010.

U.S. Department of Energy, National Nuclear Security Administration Nevada Site Office, 2011a. “Acceptance of the Area 3 Radioactive Waste Management Site Probabilistic Model, Version 2.102.” Correspondence from J. T. Carilli, NNSA/NSO, to P. M. Arnold, NSTec. August 16, 2011.

U.S. Department of Energy, National Nuclear Security Administration Nevada Site Office, 2011b. Corrective Action Decision Document/Corrective Action Plan for Corrective Action Unit 98: Frenchman Flat, Nevada National Security Site, Nevada. DOE/NV--1455. Las Vegas, NV. July 2011. 
U.S. Department of Energy, National Nuclear Security Administration Nevada Site Office, 2011c. Corrective Action Decision Document/Closure Report for Corrective Action Unit 365: Baneberry Contamination Area, Nevada National Security Site, Nevada. DOE/NV--1465. Las Vegas, NV. September 2011.

U.S. Department of Energy, National Nuclear Security Administration Nevada Site Office, 2011d. Corrective Action Decision Document/Closure Report for Corrective Action Unit 367: Area 10 Sedan, Ess and Uncle Unit Craters, Nevada National Security Site, Nevada. DOE/NV--1451. Las Vegas, NV. June 2011.

U.S. Department of Energy, National Nuclear Security Administration Nevada Site Office, 2011e. Corrective Action Decision Document/Closure Report for Corrective Action Unit 106: Areas 5, 11 Frenchman Flat Atmospheric Sites, Nevada National Security Site, Nevada. DOE/NV--1464. Las Vegas, NV. September 2011.

U.S. Department of Energy, National Nuclear Security Administration Nevada Site Office, 2011f. Corrective Action Decision Document/Corrective Action Plan for Corrective Action Unit 547: Miscellaneous Contaminated Waste Sites, Nevada National Security Site, Nevada. DOE/NV--1463. Las Vegas, NV. September 2011.

U.S. Department of Energy, National Nuclear Security Administration Nevada Site Office, 2012a. Nevada National Security Site Waste Acceptance Criteria, DOE/NV--325-Rev.9. Las Vegas, NV. February 2012.

U.S. Department of Energy, National Nuclear Security Administration Nevada Site Office, 2012b. Closure Report for the 92-Acre Area and Corrective Action Unit 111: Area 5 WMD Retired Mixed Waste Pits, Nevada National Security Site, Nevada. DOE/NV--1472. Las Vegas, NV. February 2012.

U.S. Department of Energy, National Nuclear Security Administration Nevada Site Office, 2012c. Corrective Action Decision Document for Corrective Action Unit 366: Area 11 Plutonium Valley Dispersion Sites, Nevada National Security Site, Nevada. DOE/NV--1488. Las Vegas, NV. September 2012.

U.S. Department of Energy, National Nuclear Security Administration Nevada Site Office, 2012d. Corrective Action Decision Document/Corrective Action Plan for Corrective Action Unit 104: Area 7 Yucca Flat Atmospheric Test Sites, Nevada National Security Site, Nevada. DOE/NV--1489. Las Vegas, NV. October 2012.

U.S. Department of Energy, National Nuclear Security Administration Nevada Site Office, 2012e. Corrective Action Investigation Plan for Corrective Action Unit 105: Area 2 Yucca Flat Atmospheric Test Sites, Nevada National Security Site, Nevada. DOE/NV--1486. Las Vegas, NV. September 2012. 
U.S. Department of Energy, National Nuclear Security Administration Nevada Site Office, 2012f. Corrective Action Investigation Plan for Corrective Action Unit 550: Smoky Contamination Area, Nevada National Security Site, Nevada. DOE/NV--1477. Las Vegas, NV. May 2012.

U.S. Department of Energy, National Nuclear Security Administration Nevada Site Office, 2012g. Corrective Action Investigation Plan for Corrective Action Unit 569: Area 3 Yucca Flat Atmospheric Test Sites, Nevada National Security Site, Nevada. DOE/NV--1474. Las Vegas, NV. February 2012.

U.S. Department of Energy, National Nuclear Security Administration Nevada Site Office, 2012h. Corrective Action Investigation Plan for Corrective Action Unit 570: Area 9 Yucca Flat Atmospheric Test Sites, Nevada National Security Site, Nevada. DOE/NV--1483. Las Vegas. August 2012.

U.S. Department of Energy, National Nuclear Security Administration Nevada Site Office, 2012i. Closure Report for Corrective Action Unit 547: Miscellaneous Contaminated Waste Sites, Nevada National Security Site, Nevada. DOE/NV--1480. Las Vegas, NV. July 2012.

U.S. Department of Energy, National Nuclear Security Administration Nevada Site Office, 2013. "Acceptance of the Area 5 Radioactive Waste Management Site Probabilistic Model, Version 4.114.” Correspondence from J. T. Carilli, NNSA/NSO, to P. M. Arnold, NSTec. February 21, 2013.

U.S. Environmental Protection Agency, 1999. Cancer Risk Coefficients for Environmental Exposure to Radionuclides. EPA-402-R-99-001, Supplemental CD. Washington, D.C.

U.S. Environmental Protection Agency, 2005. Revision to the Guideline on Air Quality Models: Adoption of a Preferred General Purpose Dispersion Model and Other Revisions. Federal Register, Vol. 70, No. 216, November 5, 2005. 


\section{APPENDIX A}

\section{Checklist for Review of Annual Summary}

This appendix summarizes the results of a review conducted to confirm that the annual summary contains all the information as required by the Low-Level Waste Disposal Facility Federal Review Group (LFRG) Program Management Plan.

Table A.1. Checklist for Review of Annual Summary

\begin{tabular}{|c|c|}
\hline Requirement & Result \\
\hline $\begin{array}{l}\text { 1.0 Key Questions } \\
\text { The annual summary for each disposal facility must provide } \\
\text { information sufficient to evaluate three key questions about the PA } \\
\text { for the facility: } \\
\text { a. Does the annual summary information indicate that changes } \\
\text { to the PA are required? }\end{array}$ & $\begin{array}{l}\text { A special analysis was issued for } \\
\text { the Area } 3 \text { RWMS PA in FY } 2012 . \\
\text { Section } 2.6 \text { concludes that the } \\
\text { Area } 3 \text { RWMS PA and the Area } 5 \\
\text { RWMS PA do not require revision. }\end{array}$ \\
\hline $\begin{array}{l}\text { b. Does the annual summary information indicate that the } \\
\text { conclusions of the PA remain valid? }\end{array}$ & $\begin{array}{l}\text { Section } 2.6 \text { concludes that the } \\
\text { Area } 3 \text { and Area } 5 \text { RWMS PAs } \\
\text { remain valid. }\end{array}$ \\
\hline $\begin{array}{l}\text { c. Does the annual summary information indicate that facility } \\
\text { performance will remain within the PA limits imposed by the } \\
\text { U.S. Department of Energy Manual DOE M 435.1-1 } \\
\text { performance objectives and any conditions in the facility } \\
\text { DAS? }\end{array}$ & $\begin{array}{l}\text { Section } 2.6 \text { concludes that the } \\
\text { Area } 3 \text { and Area } 5 \text { RWMSs } \\
\text { continue to meet all performance } \\
\text { objectives based on PA model } \\
\text { results using PA models updated } \\
\text { with FY } 2012 \text { data. }\end{array}$ \\
\hline $\begin{array}{l}\text { 2.0 Necessary Information } \\
\text { The information provided in the annual summary for each low-level } \\
\text { waste disposal facility should include the following: } \\
\text { a. Description of any changes affecting the PA. Does the } \\
\text { annual summary indicate whether any changes affecting the } \\
\text { PA have occurred? If so, are their effects on the PA } \\
\text { adequately described? }\end{array}$ & $\begin{array}{l}\text { Changes occurring are described } \\
\text { in Sections } 2.1 \text { through } 2.4 \text { and } \\
\text { summarized in Section 2.5. The } \\
\text { effects of changes on PA results } \\
\text { are described in Section 2.5.3. }\end{array}$ \\
\hline $\begin{array}{l}\text { Description of any PA ramifications of special analyses and } \\
\text { reviews performed or proposed for the facility. Does the } \\
\text { annual summary indicate whether any special analyses or } \\
\text { reviews were performed? If so, are the ramifications for the } \\
\text { PA adequately described? }\end{array}$ & $\begin{array}{l}\text { Special analyses for new or } \\
\text { revised waste streams are } \\
\text { described in Section } 2.1 .2 \text {. The } \\
\text { methods, results, and conclusions } \\
\text { of a special analysis conducted to } \\
\text { determine the continuing } \\
\text { adequacy of the Area } 3 \text { RWMS } \\
\text { PA are summarized in Section } \\
\text { 2.5.3. }\end{array}$ \\
\hline $\begin{array}{l}\text { c. Description of any proposed changes in facility design or } \\
\text { operations. Does the annual summary indicate whether any } \\
\text { changes are proposed in facility design or operations? If so, } \\
\text { are the effects of the proposed change on the PA } \\
\text { adequately described? }\end{array}$ & $\begin{array}{l}\text { Changes to facility designs and } \\
\text { operations are discussed in } \\
\text { Section } 2.1 \text { and } 2.2 \text {. }\end{array}$ \\
\hline $\begin{array}{l}\text { d. Description of any corresponding changes required in the } \\
\text { PA maintenance plan, the closure plan, and the monitoring } \\
\text { plan. Does the annual summary indicate whether any } \\
\text { corresponding changes are required in the plans? If so, are } \\
\text { they adequately described? }\end{array}$ & $\begin{array}{l}\text { Section } 2.5 .2 \text { concludes that no } \\
\text { changes are required for the } \\
\text { maintenance plan, closure plan, } \\
\text { or monitoring plan. }\end{array}$ \\
\hline
\end{tabular}




\begin{tabular}{|c|c|}
\hline Requirement & Result \\
\hline $\begin{array}{l}\text { e. Description of any proposed changes in the PA. Does the } \\
\text { annual summary indicate whether any changes to the } P A \\
\text { are required? If so, are they adequately described? }\end{array}$ & $\begin{array}{l}\text { Section } 2.5 .3 \text { describes proposed } \\
\text { changes to the PA model. } \\
\text { Section } 2.6 \text { concludes that no } \\
\text { changes to the PA are required. }\end{array}$ \\
\hline $\begin{array}{l}\text { 2.1 Factors to be Addressed } \\
\text { The basic factors to be addressed in the annual summary and } \\
\text { evaluated by the LFRG in reviewing the annual summary are } \\
\text { operations, facility design, closure design, and research and } \\
\text { development. More detailed descriptions of the information relevant } \\
\text { to these basic factors are provided below. (For additional detail on } \\
\text { the scope and level of detail expected for the topics, see Section } 2.2 \\
\text { of the "Maintenance Guide for U.S. Department of Energy Low-Level } \\
\text { Waste Disposal Facility Performance Assessments and Composite } \\
\text { Analyses," November 10, 1999.) } \\
\text { 2.1.1 Operations Considerations } \\
\text { Disposal unit consistency with the PA models (e.g., size and } \\
\text { configuration of trenches, shafts, and pits; waste placement and } \\
\text { configuration; thickness of operational backfill/cover). Does the } \\
\text { annual summary adequately describe disposal unit consistency with } \\
\text { the PA models? } \\
\text { a. Waste receipts including description of form and packaging } \\
\text { (especially special waste forms) and their consistency with } \\
\text { PA analyses and projections. Does the annual summary } \\
\text { adequately describe waste receipts and their consistency } \\
\text { with PA analyses and projections? }\end{array}$ & $\begin{array}{l}\text { Disposal unit design is discussed } \\
\text { in Section } 2.2 .1 \text {. Disposal unit } \\
\text { designs continue to be consistent } \\
\text { with PA models. } \\
\text { Waste receipts are described in } \\
\text { Section 2.1.2. The impacts of } \\
\text { waste receipts on PA results are } \\
\text { described in Section } 2.5 .2 \text {. }\end{array}$ \\
\hline $\begin{array}{l}\text { b. Waste acceptance criteria including radionuclides significant } \\
\text { to and evaluated in the } P A \text {, radionuclide concentration and } \\
\text { quantity limits established, waste form and packaging } \\
\text { requirements, and consistency with PA results. Does the } \\
\text { annual summary adequately describe the WAC and their } \\
\text { consistency with the PA results? }\end{array}$ & $\begin{array}{l}\text { Section } 2.1 .3 \text { describes the WAC } \\
\text { and confirms the consistency of } \\
\text { the WAC with PA results. }\end{array}$ \\
\hline $\begin{array}{l}\text { c. Procedures and systems (e.g., verification of waste } \\
\text { characteristics, inventory limit controls, generator } \\
\text { certification) intended to prevent disposal of inappropriate } \\
\text { wastes. Does the annual summary adequately describe } \\
\text { procedures and systems? }\end{array}$ & $\begin{array}{l}\text { The Radioactive Waste } \\
\text { Acceptance Program is described } \\
\text { in Section 2.1.3. }\end{array}$ \\
\hline $\begin{array}{l}\text { 2.1.2 Facility Design Considerations } \\
\text { a. Disposal technology and facility configuration consistency } \\
\text { with the PA analyses. Is the consistency adequately } \\
\text { described? }\end{array}$ & $\begin{array}{l}\text { Consistency of facility } \\
\text { configuration with PA analyses is } \\
\text { described in Section } 2.2 \text {. }\end{array}$ \\
\hline $\begin{array}{l}\text { b. Engineered barrier consistency with the PA. Is the } \\
\text { consistency adequately described? }\end{array}$ & $\begin{array}{l}\text { Consistency of engineered } \\
\text { barriers with PA analyses is } \\
\text { described in Section 2.2.2. }\end{array}$ \\
\hline $\begin{array}{l}\text { c. Monitoring provisions appropriate for evaluation of facility } \\
\text { performance. Are monitoring provisions adequately } \\
\text { described? }\end{array}$ & $\begin{array}{l}\text { The Monitoring Program is } \\
\text { described in Section } 2.2 .3 \text {. }\end{array}$ \\
\hline $\begin{array}{l}\text { d. Operational controls to promote stability and to compensate } \\
\text { for potential subsidence. Are operational controls adequately } \\
\text { described? }\end{array}$ & $\begin{array}{l}\text { Subsidence controls and } \\
\text { monitoring methods are described } \\
\text { in Section 2.2.4. }\end{array}$ \\
\hline
\end{tabular}




\begin{tabular}{|c|c|}
\hline Requirement & Result \\
\hline $\begin{array}{l}\text { 2.1.3 Closure Design Considerations } \\
\text { a. Engineered barrier description including consistency of the } \\
\text { closure cover design with PA analysis and threats to cover } \\
\text { integrity and viability. Are engineered barriers adequately } \\
\text { described? }\end{array}$ & $\begin{array}{l}\text { Closure cover design and } \\
\text { consistency with PA analyses are } \\
\text { described in Section 2.3. }\end{array}$ \\
\hline $\begin{array}{l}\text { b. Future land-use plan consistency with PA assumptions. Is } \\
\text { consistency of the land-use plan with the PA assumptions } \\
\text { adequately described? }\end{array}$ & $\begin{array}{l}\text { Land-use plan consistency with } \\
\text { PA assumptions is described in } \\
\text { Section 2.3.2. }\end{array}$ \\
\hline $\begin{aligned} 2.1 .4 & \text { Research and Development Considerations } \\
\text { a. } & \text { R\&D efforts required by the facility disposal authorization } \\
& \text { statement. Are these efforts adequately described? }\end{aligned}$ & $\begin{array}{l}\text { R\&D efforts required by the } \\
\text { Area } 3 \text { RWMS and Area } 5 \text { RWMS } \\
\text { DASs are summarized in } \\
\text { Section } 1.1, \text { Tables } 1 \text { and } 3 \text {, } \\
\text { respectively. The tables' status } \\
\text { column indicates that all } \\
\text { conditions were closed in } 2002 \text {. }\end{array}$ \\
\hline $\begin{array}{l}\text { b. R\&D efforts pursued for improving and refining the performance } \\
\text { assessment. Are these efforts adequately described? }\end{array}$ & $\begin{array}{l}\text { R\&D efforts required by PA/CA } \\
\text { reviews and their resolution are } \\
\text { summarized in Section } 1.2 \text {, } \\
\text { Table } 5 \text {. Ongoing R\&D efforts are } \\
\text { described in Section } 2.4 \text {. }\end{array}$ \\
\hline $\begin{array}{l}\text { c. Results of any confirmatory testing performed. Was any } \\
\text { confirmatory testing performed? If so, are the results } \\
\text { adequately described? }\end{array}$ & $\begin{array}{l}\text { Confirmatory monitoring of site } \\
\text { performance is described under } \\
\text { monitoring in Section } 2.2 .3 \text {. } \\
\text { Monitoring results are published } \\
\text { elsewhere as cited in the text. }\end{array}$ \\
\hline $\begin{array}{l}\text { Changes } \\
\text { The changes that could cause divergence from the conditions used } \\
\text { for the PA analysis should be categorized as discovered changes, } \\
\text { proposed changes, or R\&D changes and should be listed and } \\
\text { described in the annual summary. } \\
\text { [Note: This section of the review should focus on description of the } \\
\text { changes (discovered, proposed, and R\&D) and any effects of the } \\
\text { changes not described in Section 2.2.] } \\
2.2 .1 \text { Discovered Changes } \\
\text { The annual summary should report divergences from expected or } \\
\text { planned conditions that have been discovered in facility operations, } \\
\text { construction, site characteristics, and other conditions significant to } \\
\text { facility performance. Specific information should address the } \\
\text { baseline from which the divergence was identified, comparison of } \\
\text { expected conditions to any available monitoring results, significance } \\
\text { of the divergence as indicated by comparison to the four LFRG } \\
\text { review thresholds (listed below), and incorporation of the changes in } \\
\text { the performance assessment, if appropriate. } \\
\text { The four LFRG review thresholds that trigger the review by the } \\
\text { LFRG are } \\
\text { a. an increase of } 25 \text { percent or more in the forecasted doses } \\
\text { reported in the current, approved facility documentation or } \\
\text { any violation of the performance objectives imposed by } \\
\text { DOE M } 435.1-1 \text {, }\end{array}$ & $\begin{array}{l}\text { Section } 2.5 .3 \text { summarizes the } \\
\text { FY } 2012 \text { PA results for the Area } 3 \\
\text { and Area } 5 \text { RWMSs. Changes are } \\
\text { noted. All results continue to meet } \\
\text { all performance objectives. } \\
\text { The Area } 3 \text { RWMS all-pathways } \\
\text { dose and }{ }^{222} \mathrm{Rn} \text { flux density have } \\
\text { increased relative to the PA but } \\
\text { remain a small fraction of the } \\
\text { performance objective. } \\
\text { The Area } 5 \text { RWMS }{ }^{222} \text { Rn flux } \\
\text { density increased more than } 25 \% \\
\text { between FY } 2011 \text { and FY } 2012 \\
\text { due to inventory increases. The } \\
95^{\text {th }} \text { percentile flux is less than the } \\
\text { performance objective. }\end{array}$ \\
\hline
\end{tabular}




\begin{tabular}{|c|c|}
\hline Requirement & Result \\
\hline $\begin{array}{l}\text { b. any change in the point of compliance as reported in the } \\
\text { current approved facility documentation, }\end{array}$ & $\begin{array}{l}\text { Changes to PA models are } \\
\text { described in Section } 2.4 \text {. No } \\
\text { change in the point of compliance } \\
\text { occurred in FY } 2012 \text {. }\end{array}$ \\
\hline $\begin{array}{l}\text { c. any fundamental change in the analysis methodology or } \\
\text { model used for the facility documentation, and }\end{array}$ & $\begin{array}{l}\text { Changes to PA models are } \\
\text { described in Section } 2.4 .\end{array}$ \\
\hline $\begin{array}{l}\text { d. any fundamental change in the hydrologic or geologic } \\
\text { parameters used in the facility analysis methodology or model. }\end{array}$ & $\begin{array}{l}\text { Changes to PA models are } \\
\text { described in Section } 2.4 \text {. There } \\
\text { are no changes in hydrologic or } \\
\text { geologic models. }\end{array}$ \\
\hline $\begin{array}{l}\text { 2.2.2 Proposed Changes } \\
\text { a. The annual summary should identify divergences from } \\
\text { expected or planned conditions that have been or will be } \\
\text { voluntarily made by the facility operators to facility operations, } \\
\text { facility construction, or other conditions significant to facility } \\
\text { performance. Specific information should address the } \\
\text { baseline from which the divergence is planned, comparison of } \\
\text { current performance to performance expected after the } \\
\text { change is made, significance of the divergence as indicated } \\
\text { by comparison to the four LFRG review thresholds (listed in } \\
\text { Section } 2.4 .1 \text { above), and incorporation of the changes in the } \\
\text { performance assessment, if appropriate. Does the annual } \\
\text { summary report any proposed changes? If so, are they } \\
\text { adequately described? }\end{array}$ & $\begin{array}{l}\text { Proposed changes are described } \\
\text { in Section 2.5.2. }\end{array}$ \\
\hline $\begin{array}{l}\text { 2.2.3 Research and Development Changes } \\
\text { a. The annual summary should include descriptions of } \\
\text { research and development (both generic and site-specific) } \\
\text { relevant to the PA analysis models and input data for them } \\
\text { that are to be used to improve the conclusions of the PA. } \\
\text { The annual summary should include a description of the } \\
\text { significance of the improvements, when and how the } \\
\text { anticipated improvements will be incorporated in PA } \\
\text { modeling and analyses, and whether the improvements are } \\
\text { expected to change the conclusions of the PA. Does the } \\
\text { annual summary report any R\&D changes? If so, are they } \\
\text { adequately described? }\end{array}$ & $\begin{array}{l}\text { R\&D changes are described in } \\
\text { Section } 2.4 \text {. The effects of } \\
\text { changes to the PA models are } \\
\text { described in Section } 2.5 .3 \text {. }\end{array}$ \\
\hline $\begin{array}{l}\text { 3.0 Composite Analysis Summary } \\
\text { The annual summary for each disposal facility should provide the } \\
\text { information required by the LFRG members and staff to evaluate } \\
\text { whether the facility CA continues to satisfy the requirements of } \\
\text { DOE M 435.1-1 and any additional conditions specified in the facility } \\
\text { disposal authorization statement. The focus of the CA review will be } \\
\text { on the interacting source terms relative to the performance goals } \\
\text { established in DOE M } 435.1-1 \text { because the review of the facility PA } \\
\text { is focused on the facility itself. } \\
\text { a. Does the annual summary state that the conclusions of the } \\
\text { CA remain valid? If so, does the annual summary state } \\
\text { whether confidence in the conclusions has changed? }\end{array}$ & $\begin{array}{l}\text { Section } 3.5 \text { concludes that the } \\
\text { Area } 3 \text { and Area } 5 \text { RWMS CAs } \\
\text { remain valid and that there } \\
\text { continues to be a high likelihood } \\
\text { of compliance with the } 0.3 \mathrm{mSv} \\
\text { dose constraint. }\end{array}$ \\
\hline
\end{tabular}




\begin{tabular}{l} 
Requirement \\
\hline 3.1 Key Questions \\
The annual summary for each disposal facility must provide \\
information sufficient to evaluate three key questions about the \\
composite analysis for the facility: \\
a. Does the annual summary information indicate that changes \\
to the CA are required?
\end{tabular}

$b$. Does the annual summary information indicate that the conclusions of the $C A$ remain valid?

c. Does the annual summary information indicate that the facility performance will remain within the CA performance goals provided in the DOE M 435.1-1 performance goals and any conditions in the facility DAS?

\subsection{Necessary Information}

[This section of the review should focus on the effects of the changes on the CA. Section 3.4 should focus on description of the changes and any effects not described in this section.]

The information provided in the annual summary for each low-level waste disposal facility should include the following:

a. Description of any changes affecting the CA including changes in the design or operations of facilities with releases potentially interacting with the disposal facility releases. Does the annual summary indicate whether any changes affecting the CA have occurred? If so, are their effects on the $C A$ adequately described?

b. Description of any CA ramifications of special analyses and reviews performed or proposed for the facility. Does the annual summary indicate whether any special analyses or reviews were performed? If so, are the ramifications for the $C A$ adequately described?

c. A description of any proposed changes in the low-level waste disposal facility design or operations. Does the annual summary indicate whether any changes are proposed in facility design or operations? If so, are the effects of the proposed changes on the CA adequately described?

d. A description of proposed changes (including remediation activities) in design or operations of facilities with releases potentially interacting with the disposal facility releases. Does the annual summary indicate whether any changes are proposed in the design or operations of facilities with releases potentially interacting with the disposal facility? If so, are the effects of the proposed changes on the $C A$ adequately described?
Result

Section 3.5 concludes that no changes or revisions to the CAs are required.
Section 3.5 concludes that the conclusions of the CAs remain valid.

Section 3.5 concludes that there is a reasonable expectation that the Area 3 and Area 5 RWMSs meet the $0.3 \mathrm{mSv}$ dose constraint.

The pre-1988 RWMS disposal units are the only facilities interacting with the RWMSs. RWMS design and operations changes affecting the CAs are described in Section 3.1. The effects of all changes on the CA results are described in Section 3.4.3.
Section 3.4.3 describes the Area 3 RWMS special analysis performed to assess the continuing adequacy of the CA. The effects of changes on CA results are assessed using the current CA model. Current CA results are included in Section 3.4.3.

Section 3.1 describes RWMS facility changes occurring in FY 2012. No significant changes to pre-1988 disposal units occurred at the Area 3 RWMS and Area 5 RWMS in FY 2012. Changes in facilities (the RWMSs) are summarized in Section 3.1.1. Changes in interacting Environmental Restoration sites are summarized in Section 3.3. Changes in Environmental Restoration sites due to completed and planned corrective actions are not expected to affect CA results. 


\begin{tabular}{|c|c|}
\hline Requirement & Result \\
\hline $\begin{array}{l}\text { A description of any corresponding changes required in the } \\
\text { CA maintenance plan, the closure plan, and the monitoring } \\
\text { plan. Does the annual summary indicate whether any } \\
\text { corresponding changes are required in the plans? If so, are } \\
\text { they adequately described? }\end{array}$ & $\begin{array}{l}\text { Section } 3.4 .2 \text { states that there are } \\
\text { no recommended changes to the } \\
\text { maintenance plan, monitoring } \\
\text { plan, and closure plan. }\end{array}$ \\
\hline $\begin{array}{l}\text { f. A description of any proposed changes in the CA. Does the } \\
\text { annual summary indicate whether any changes to the CA } \\
\text { are required? If so, are they adequately described? }\end{array}$ & $\begin{array}{l}\text { Proposed changes are } \\
\text { summarized in Section } 3.4 .2 \text {. } \\
\text { Section } 3.5 \text { concludes that no } \\
\text { changes to the CAs are required. }\end{array}$ \\
\hline \multicolumn{2}{|l|}{$\begin{array}{l}\text { 3.3 Factors to be Addressed } \\
\text { The basic factors to be addressed in the annual summary and } \\
\text { evaluated by the LFRG in reviewing the annual summary are } \\
\text { operations, facility design, closure design, research and } \\
\text { development, and interacting source terms. (For additional detail on } \\
\text { the scope and level of detail expected for the topics, see Section } 2.2 \\
\text { of the "Maintenance Guide for U.S. Department of Energy Low-Level } \\
\text { Waste Disposal Facility Performance Assessments and Composite } \\
\text { Analyses," November 10, 1999.) }\end{array}$} \\
\hline $\begin{array}{l}\text { 3.3.1 Operations Considerations } \\
\text { a. Significant changes in the operations (including remediation } \\
\text { activities) and configurations of facilities with releases that } \\
\text { could potentially interact with releases from the low-level } \\
\text { waste disposal facility. Does the annual summary describe } \\
\text { any significant changes in potentially interacting facilities? }\end{array}$ & $\begin{array}{l}\text { Section } 3.1 \text { describes changes to } \\
\text { the RWMSs operations and } \\
\text { configuration. Section } 3.3 \\
\text { describes changes to interacting } \\
\text { Environmental Restoration } \\
\text { sources affecting the CAs. }\end{array}$ \\
\hline $\begin{array}{l}\text { b. Disposal unit consistency with the CA models (e.g., size and } \\
\text { configuration of trenches, shafts, and pits; waste placement } \\
\text { and configuration; thickness of operational backfill/cover). } \\
\text { Does the annual summary adequately describe disposal unit } \\
\text { consistency with the CA models? }\end{array}$ & $\begin{array}{l}\text { Section } 3.1 .1 \text { describes RWMSs } \\
\text { disposal unit changes affecting } \\
\text { the CAs. }\end{array}$ \\
\hline $\begin{array}{l}\text { c. Waste receipts including description of form and packaging } \\
\text { (especially special waste forms) and their consistency with } \\
\text { CA analyses and projections. Does the annual summary } \\
\text { adequately describe waste receipts and their consistency } \\
\text { with CA analyses and projections? }\end{array}$ & $\begin{array}{l}\text { Section } 3.1 .1 .1 \text { describes } \\
\text { changes to the pre-1988 waste } \\
\text { inventories. Changes to } \\
\text { post-1988 inventories are } \\
\text { described in Section 2.1.2. }\end{array}$ \\
\hline $\begin{array}{l}\text { d. Waste acceptance criteria including radionuclides significant } \\
\text { to and evaluated in the CA, radionuclide concentration and } \\
\text { quantity limits (established in the PA), and waste form and } \\
\text { packaging requirements. Does the annual summary } \\
\text { adequately describe the WAC and their consistency with the } \\
\text { CA results? }\end{array}$ & $\begin{array}{l}\text { The WAC are described in } \\
\text { Section 2.1.3. }\end{array}$ \\
\hline $\begin{array}{l}\text { Procedures and systems (e.g., verification of waste } \\
\text { characteristics, inventory limit controls, generator } \\
\text { certification) intended to prevent disposal of inappropriate } \\
\text { wastes. Does the annual summary adequately describe } \\
\text { procedures and systems? }\end{array}$ & $\begin{array}{l}\text { The Radioactive Waste } \\
\text { Acceptance Program is described } \\
\text { in Section 2.1.3. }\end{array}$ \\
\hline $\begin{array}{ll}\text { 3.3.2 Facility Design Considerations } \\
\text { a. } \\
\text { and consistency with the CA analyses of operations technology } \\
\text { interacting with releases from the low-level waste disposal } \\
\text { facility. Is the consistency adequately described? }\end{array}$ & $\begin{array}{l}\text { Consistency of facility design with } \\
\text { CA analyses is described in } \\
\text { Section 3.1.1. }\end{array}$ \\
\hline
\end{tabular}




\begin{tabular}{|c|c|}
\hline Requirement & Result \\
\hline $\begin{array}{l}\text { b. Engineered barrier consistency the CA. Is the consistency } \\
\text { adequately described? }\end{array}$ & $\begin{array}{l}\text { Consistency of facility design with } \\
\text { CA analyses is described in } \\
\text { Section 3.1.1.1. Consistency of } \\
\text { cover design with CA analyses is } \\
\text { described in Section 3.1.1.3. }\end{array}$ \\
\hline $\begin{array}{l}\text { c. Monitoring provisions appropriate for evaluation of facility } \\
\text { performance and interacting source terms. Are monitoring } \\
\text { provisions adequately described? }\end{array}$ & $\begin{array}{l}\text { The CA monitoring program is } \\
\text { described in Section 3.1.1.2. }\end{array}$ \\
\hline $\begin{array}{l}\text { d. Operational controls to promote stability and to compensate } \\
\text { for potential subsidence. Are operational controls adequately } \\
\text { described? }\end{array}$ & $\begin{array}{l}\text { Controls and monitoring of } \\
\text { subsidence are described in } \\
\text { Section 2.2.4. }\end{array}$ \\
\hline $\begin{array}{l}\text { 3.3.3 Closure Design Considerations } \\
\text { a. Engineered barrier description (including those for facilities } \\
\text { with releases that interact with the low-level waste disposal } \\
\text { facility) including consistency of the closure cover design } \\
\text { with CA analysis and threats to cover integrity and viability. } \\
\text { Are engineered barriers adequately described? }\end{array}$ & $\begin{array}{l}\text { Consistency of disposal unit cover } \\
\text { design with CA analyses is } \\
\text { described in Section 3.1.1.3. } \\
\text { Consistency of Environmental } \\
\text { Restoration closures with CA } \\
\text { analyses is described in } \\
\text { Section 3.3. }\end{array}$ \\
\hline $\begin{array}{l}\text { b. Future land-use plan consistency with CA assumptions. Is } \\
\text { consistency of the land-use plan with the CA assumptions } \\
\text { adequately described? }\end{array}$ & $\begin{array}{l}\text { The consistency of land-use plans } \\
\text { with CA assumptions is discussed } \\
\text { in Section 3.3. }\end{array}$ \\
\hline $\begin{array}{ll}\text { 3.3.4 Research and Development Considerations } \\
\text { a. } R \& D \text { efforts required by the DAS. Are these efforts } \\
\text { adequately described? }\end{array}$ & $\begin{array}{l}\text { R\&D efforts required by the } \\
\text { Area } 3 \text { RWMS and Area } 5 \text { RWMS } \\
\text { DASs are summarized in } \\
\text { Section 1.1, Tables } 1 \text { and } 3 \text {, } \\
\text { respectively. The tables' status } \\
\text { column indicates that all } \\
\text { conditions were closed in } 2002 \text {. } \\
\text { R\&D efforts relevant to the CAs } \\
\text { are described in Section } 3.2 \text {. } \\
\text { DAS-required R\&D efforts to } \\
\text { characterize UGTA source terms } \\
\text { are described in Section 3.3.1. }\end{array}$ \\
\hline $\begin{array}{l}\text { b. R\&D efforts pursued for improving and refining the } \\
\text { composite analysis. Are these efforts adequately described? }\end{array}$ & $\begin{array}{l}\text { R\&D efforts relevant to the CAs } \\
\text { are described in Section } 3.2 \text {. }\end{array}$ \\
\hline $\begin{array}{l}\text { c. Results of any confirmatory testing performed. Was any } \\
\text { confirmatory testing performed? If so, are the results } \\
\text { adequately described? }\end{array}$ & $\begin{array}{l}\text { Confirmatory monitoring is } \\
\text { described in Section 3.1.1.2. }\end{array}$ \\
\hline $\begin{array}{l}\text { 3.3.5 Interacting Source Term Considerations } \\
\text { a. Evaluation of significant interacting source terms. Does the } \\
\text { annual summary indicate that there is a need to re-evaluate } \\
\text { significant interacting source terms? If so, are they } \\
\text { adequately re-evaluated? }\end{array}$ & $\begin{array}{l}\text { Section } 3.3 \text { reviews the status of } \\
\text { interacting source terms and } \\
\text { concludes that no significant } \\
\text { changes have occurred for the } \\
\text { Area } 3 \text { RWSM and Area } 5 \\
\text { RWMS. }\end{array}$ \\
\hline $\begin{array}{l}\text { b. Alteration of existing source terms. Does the annual } \\
\text { summary report any changes in existing source terms } \\
\text { including new source terms? }\end{array}$ & $\begin{array}{l}\text { Section } 3.3 \text { reviews corrective } \\
\text { action investigations and } \\
\text { corrective actions affecting } \\
\text { interacting source terms and } \\
\text { concludes that no significant } \\
\text { changes have occurred for the } \\
\text { Area } 3 \text { RWMS and Area } 5 \text { RWMS. }\end{array}$ \\
\hline
\end{tabular}




\begin{tabular}{|c|c|}
\hline Requirement & Result \\
\hline $\begin{array}{l}\text { c. Alteration of uncertainty in characteristics of existing } \\
\text { sources. Does the annual summary report any changes in } \\
\text { uncertainty in characteristics of existing source terms? }\end{array}$ & $\begin{array}{l}\text { Section } 3.3 \text { reviews the status of } \\
\text { interacting source terms and } \\
\text { concludes that there is no } \\
\text { significant change in existing } \\
\text { source term uncertainty. }\end{array}$ \\
\hline $\begin{array}{l}\text { Changes } \\
\text { The changes that could cause divergence from the conditions used } \\
\text { for the CA analysis should be categorized as discovered changes, } \\
\text { proposed changes, or R\&D changes and should be listed and } \\
\text { described in the annual summary. } \\
\text { [This section of the review should focus on description of the } \\
\text { changes (discovered, proposed, and R\&D) and any effects of the } \\
\text { changes not described in Section 3.2.] } \\
\text { 3.4.1 Discovered Changes } \\
\text { The annual summary should report divergences from expected or } \\
\text { planned conditions that have been discovered in facility operations, } \\
\text { construction, site characteristics, and other conditions significant to } \\
\text { determination of cumulative doses from the disposal facility and } \\
\text { potentially interacting source terms. Specific information should } \\
\text { address the baseline from which the divergence was identified, } \\
\text { comparison of expected conditions to any available monitoring } \\
\text { results, significance of the divergence as indicated by comparison to } \\
\text { the four LFRG review thresholds (listed in Section } 2.4 .1 \text { above), and } \\
\text { incorporation of the changes in the performance assessment, if } \\
\text { appropriate. } \\
\text { a. Does the annual summary report any discovered changes? } \\
\quad \text { If so, are they adequately described? }\end{array}$ & $\begin{array}{l}\text { Section } 3.4 .1 \text { describes } \\
\text { discovered changes affecting the } \\
\text { CA. The Area } 3 \text { RWMS has two } \\
\text { discovered changes for } \\
\text { interacting sources. The changes } \\
\text { have no significant effect on CA } \\
\text { results. The Area } 5 \text { RWMS has no } \\
\text { discovered changes. }\end{array}$ \\
\hline $\begin{array}{l}\text { 3.4.2 Proposed Changes } \\
\text { a. The annual summary should identify divergences (for both } \\
\text { the low-level waste disposal facility and for facilities with } \\
\text { potentially interacting source terms) from expected or } \\
\text { planned conditions that have been or will be voluntarily } \\
\text { made by the facility operators to facility operations, facility } \\
\text { construction, interacting source terms, or other conditions } \\
\text { significant to combined facility and interacting source } \\
\text { behavior. Specific information should address the baseline } \\
\text { from which the divergence is planned, comparison of current } \\
\text { performance to performance expected after the change is } \\
\text { made, significance of the divergence as indicated by } \\
\text { comparison to the four LFRG review thresholds (listed in } \\
\text { Section } 2.4 .1 \text { above), and incorporation of the changes in } \\
\text { the performance assessment, if appropriate. Does the } \\
\text { annual summary report any proposed changes? If so, are } \\
\text { they adequately described? }\end{array}$ & $\begin{array}{l}\text { Proposed changes to the CAs are } \\
\text { described in Section 3.4.2. The } \\
\text { effects of changes on CA results } \\
\text { are presented and discussed in } \\
\text { Section 3.4.3. }\end{array}$ \\
\hline
\end{tabular}




\begin{tabular}{|c|c|c|}
\hline \multicolumn{2}{|c|}{ Requirement } & \multirow[b]{2}{*}{$\begin{array}{l}\text { Result } \\
\text { The CA R\&D efforts are described } \\
\text { in Section 3.2. The significance } \\
\text { and effect of R\&D changes on CA } \\
\text { results are described in } \\
\text { Section 3.4.3. }\end{array}$} \\
\hline $\begin{array}{r}3.4 .3 \\
a\end{array}$ & $\begin{array}{l}\text { Research and Development Changes } \\
\text { The annual summary should include descriptions of } \\
\text { research and development (both generic and site-specific) } \\
\text { relevant to the CA analysis models and input data for them } \\
\text { that are to be used to improve the conclusions of the CA. } \\
\text { The annual summary should include description of the } \\
\text { significance of the improvements, when and how the } \\
\text { anticipated improvements will be incorporated in CA } \\
\text { modeling and analyses, and whether the improvements are } \\
\text { expected to change the conclusions of the CA. Does the } \\
\text { annual summary report any R\&D changes? If so, are they } \\
\text { adequately described? }\end{array}$ & \\
\hline $\begin{array}{r}4.0 \\
\quad a\end{array}$ & $\begin{array}{l}\text { Disposal Authorization Statements } \\
\text { The facility annual summary should describe the conditions } \\
\text { stated in the current DAS for the facility. For conditions that } \\
\text { specify actions to be taken (such as resolution of data } \\
\text { uncertainties), the annual summary should describe the } \\
\text { required action, any deadlines specified in the DAS, and the } \\
\text { current status of efforts to satisfy the requirement. For } \\
\text { conditions that place limits on the operations of a facility } \\
\text { (such as the maximum allowable inventory of a specified } \\
\text { radionuclide), the annual summary should describe the limit, } \\
\text { actions taken to ensure compliance with the limit, and either } \\
\text { a statement of compliance with the limit or a description and } \\
\text { explanation of any divergence. Does the annual summary } \\
\text { state whether any DAS conditions are in effect? If so, are } \\
\text { they adequately described including satisfaction of any } \\
\text { continuing limitations and description of actions to resolve } \\
\text { temporary conditions? }\end{array}$ & $\begin{array}{l}\text { The DAS and closure of all DAS } \\
\text { conditions in } 2002 \text { are discussed } \\
\text { in Section } 1.1 \text {. Minor issues being } \\
\text { addressed by the PA/CA } \\
\text { maintenance process are } \\
\text { described in Section } 1.2 \text {. }\end{array}$ \\
\hline $\begin{array}{l}5.0 \\
\text { The a } \\
\text { maint } \\
\text { descr } \\
\text { or fine } \\
\text { docur } \\
\text { and } s \\
\text { shoul } \\
\text { Is the } \\
\text { milest } \\
\text { and a }\end{array}$ & $\begin{array}{l}\text { Status of Other Required Documents } \\
\text { nual summary should describe the status of the facility PA/CA } \\
\text { nance plan, the monitoring plan, and the closure plan. The } \\
\text { tion should state whether the documents are currently in draft } \\
\text { form and should describe any planned revisions. For } \\
\text { ents that are in draft form, a description of the key milestones } \\
\text { hedule for completion should be provided. Complete citations } \\
\text { be provided for the current version (or draft) of each document. } \\
\text { tatus of the documents adequately described including } \\
\text { nes and schedules for completion of any that are in draft form, } \\
\text { full citations provided for the required documents? }\end{array}$ & $\begin{array}{l}\text { The final Maintenance Plan, } \\
\text { Closure Plans, and Monitoring } \\
\text { Plans are identified in } \\
\text { Sections } 1.2,2.3 .1 \text {, and } 2.2 .3 \text {, } \\
\text { respectively. Complete citations } \\
\text { are found in Section } 4.0 \text {. }\end{array}$ \\
\hline
\end{tabular}

CA Composite Analysis

DAS Disposal Authorization Statement

DOE U.S. Department of Energy

FY fiscal year

LFRG Low-Level Waste Disposal Facility Federal Review Group

$\mathrm{mSv}$ millisievert(s)

PA Performance Assessment

R\&D Research and Development

RWMS Radioactive Waste Management Site

UGTA Underground Test Area

WAC Waste Acceptance Criteria 
This Page Intentionally Left Blank 


\section{DISTRIBUTION}

Jhon T. Carilli

$1 \mathrm{CD}$

LLW Federal Sub-Project Director, Waste Management Project

U.S. Department of Energy

National Nuclear Security Administration

Nevada Site Office

P.O. Box 98518, M/S 505

Las Vegas, NV 89193-8518

U.S. Department of Energy

2 CDs

National Nuclear Security Administration

Nevada Site Office

Public Reading Facility

P.O. Box 98521, M/S 400

Las Vegas, NV 89193-8521

U.S. Department of Energy

1 digital file

Office of Scientific and Technical Information

P.O. Box 62

Oak Ridge, TN 37831-0062

Irene Farnham

1 copy

Navarro-Intera, LLC

P.O. Box 98592, M/S 505

Las Vegas, NV 89193-8518

David B. Hudson

1 copy

National Security Technologies, LLC

P.O. Box 98521, M/S NLV083

Las Vegas, NV 89193-8521

Susan K. Krenzien

1 copy

Navarro-Intera, LLC

P.O. Box 98592, M/S 505

Las Vegas, NV 89193-8518

Gregory J. Shott

1 copy

National Security Technologies, LLC

P.O. Box 98521, M/S NLV083

Las Vegas, NV 89193-8521 
Vefa Yucel 1 copy

National Security Technologies, LLC

P.O. Box 98521, M/S NLV083

Las Vegas, NV 89193-8521 